\title{
Local quantum constraints
}

\author{
HendRik GRundLing \\ Department of Mathematics, \\ University of New South Wales, \\ Sydney, NSW 2052, Australia. \\ hendrik@maths.unsw.edu.au
}

\author{
FERNANDO LLEDÓ* \\ Max-Planck-Institut für Gravitationsphysik, \\ Albert-Einstein-Institut, \\ Am Mühlenberg 1, \\ D-14476 Golm, Germany. \\ lledo@aei-potsdam.mpg.de
}

AMS classification: 81T05, 81T10, 46L60, 46N50

\begin{abstract}
We analyze the situation of a local quantum field theory with constraints, both indexed by the same set of space-time regions. In particular we find "weak" Haag-Kastler axioms which will ensure that the final constrained theory satisfies the usual Haag-Kastler axioms. GuptaBleuler electromagnetism is developed in detail as an example of a theory which satisfies the "weak" Haag-Kastler axioms but not the usual ones. This analysis is done by pure $\mathrm{C}^{*_{-}}$ algebraic means without employing any indefinite metric representations, and we obtain the same physical algebra and positive energy representation for it than by the usual means. The price for avoiding the indefinite metric, is the use of nonregular representations and complex valued test functions. We also exhibit the precise connection with the usual indefinite metric representation.

We conclude the analysis by comparing the final physical algebra produced by a system of local constrainings with the one obtained from a single global constraining and also consider the issue of reduction by stages. For the usual spectral condition on the generators of the translation group, we also find a "weak" version, and show that the Gupta-Bleuler example satisfies it.
\end{abstract}

\section{Introduction}

In many quantum field theories there are constraints consisting of local expressions of the quantum fields, generally written as a selection condition for the physical subspace $\mathcal{H}^{(p)}$. In the physics literature the selection condition usually takes the form:

$$
\mathcal{H}^{(p)}:=\left\{\psi \mid \chi(x) \psi=0 \quad \forall x \in \mathbb{R}^{4}\right\}
$$

where $\chi$ is some operator-valued distribution (so more accurately $\chi(x) \psi=0$ should be written as $\chi(f) \psi=0$ for all test functions $f$ ). Since the constraints $\chi$ are constructed from the smeared quantum fields, one expects them to have the same net structure in space-time as the smeared quantum fields. The question then arises as to how locality properties and constraining intertwines. This question will be at the focus of our interest in this paper.

To properly study locality questions, we shall use algebraic quantum field theory, a welldeveloped theory built on a net of $\mathrm{C}^{*}$-algebras satisfying the Haag-Kastler axioms [1, 2], but we shall assume in addition a local net of constraints (to be defined in Section 3). To impose these

* On leave from: Mathematical Institute, University of Potsdam, Am Neuen Palais 10, Postfach 601 553, D14415 Potsdam, Germany. E-mail: lledo@rz.uni-potsdam.de 
constraints at the algebraic level, we use the method developed by Grundling and Hurst [3], and this can be done either in each local algebra separately or globally in the full field algebra. We will compare the results of these two different routes, and will find conditions on the local net of constraints to ensure that the net of algebras obtained after constraining satisfies the Haag-Kastler axioms. In fact one can weaken the Haag-Kastler axioms on the original system, providing that after constraining the final net obtained satisfies these axioms. We characterise precisely what these "weak" Haag-Kastler axioms are. In our subsequent example (Gupta-Bleuler electromagnetism) we find that this weakening is crucial, since the original constraints violate the causality axiom. In our example we will avoid the usual indefinite metric representations, but will obtain by $\mathrm{C}^{*}$-algebraic means both the correct physical algebra, and the same (positive energy) representation than the one produced via the indefinite metric. Thus we show that a gauge quantum field can be completely described by $\mathrm{C}^{*}$-algebraic means, in a framework of Algebraic QFT, without the use of indefinite metric representations. There is however a cost for avoiding the indefinite metric, and this consists of the use of nonregular representations, and the use of complex valued test functions (this is related to causality violation). Fortunately, both of these pathologies only involve nonphysical parts of the theory which are eliminated after constraining, thus the final theory is again well-behaved.

Since local constraints are usually generators of gauge transformations of the second kind, the theory developed here can be considered as complementing the deep Doplicher-Haag-Roberts analysis of systems with gauge transformations of the first kind [4]. Our axioms will be slightly different (weakened Haag-Kastler axioms), and we will work with an abstract net of $\mathrm{C}^{*}$-algebras, whereas the DHR analysis is done concretely in a positive energy representation.

We need to remark that there is a range of reduction algorithms for quantum constraints available in the literature, cf. [5] at different degrees of rigour. These involve either more structure and choices, or are representation dependent, or the maps involved have pathologies from the point of view of $\mathrm{C}^{*}$-algebras. That is why we chose the method of [3]. Furthermore, the HaagKastler axioms have not previously been included in any of the reduction techniques in [5]. Locality has been examined in specific constrained theories in the literature (cf. [6], [7]) but not in the general terms we do here.

The architecture of the paper is as follows. In Section 2 we collect general facts of the constraint procedure of [3] which we will need in the subsequent sections. There is some new material in this section, since we need to extend the previous method to cover the current situation. In Section 3 we introduce our basic object, a "system of local quantum constraints" as well as the "weak Haag-Kastler axioms" and prove that after local constraining of such a system, we obtain a system satisfying the Haag-Kastler axioms. Section 4 consists of some preliminary material necessary for the development of our example in Section 5, Gupta-Bleuler electromagnetism, ( $\mathrm{C}^{*}$-algebra version adapted from [8]) and we verify all the weak Haag-Kastler axioms for it. We concretely characterize the net of constrained algebras, but it turns out that in order to obtain a simple global algebra we need to do a second stage of constraining (traditionally thought of as imposing the Maxwell equations, but here it is slightly stronger than that). We also verify the weak Haag-Kastler axioms for this second stage of constraints, and we work out in detail the connection of the $\mathrm{C}^{*}$-theory with the usual indefinite metric representation. In Section 6 we consider miscellaneous topics raised by the previous Sections. First, for a system of local constraints, we consider the relation between the algebras obtained from a single global constraining, and the inductive limit of the algebras found from local constrainings. We show that for the Gupta-Bleuler example these two algebras are the same. Secondly, we develop the theory of constraint reduction by stages (i.e. impose the constraints sequentially along an increasing chain of subsets instead of all at once). We then show that the two-step reduction procedure of the Gupta-Bleuler example satisfies this criterion. Thirdly, we consider the spectral condition (on the generators of translations) which occur in Haag-Kastler theory, and find a "weak" version 
of it which will guarantee that the final constrained theory satisfies the usual spectral condition. We show that the Gupta-Bleuler example satisfies it, by demonstrating that from the indefinite metric in the heuristic theory we can define a (positive metric) representation of the constrained algebra which satisfies the spectral condition. There are two appendices containing additional constraining facts needed in proofs, and one long proof which would have interrupted the flow of the paper.

\section{Kinematics for Quantum Constraints.}

In this section we present the minimum preliminary material necessary to define our primary problem. The reader whose main interest is quantum constraints will find this section interesting in its own right, as well as many general constraint results scattered throughout the paper. Here we present a small generalisation of the T-procedure of Grundling and Hurst [3, 8]. All new results will be proven here and for other proofs we refer to the literature.

In heuristic physics a set of constraints is a set $\left\{A_{i} \mid i \in I\right\}$ (with $I$ an index set) of operators on some Hilbert space together with a selection condition for the subspace of physical vectors:

$$
\mathcal{H}^{(p)}:=\overline{\left\{\psi \mid A_{i} \psi=0 \quad \forall i \in I\right\}} .
$$

The set of traditional observables is then the commutant $\left\{A_{i} \mid i \in I\right\}^{\prime}$ which one can enlarge to the set of all observables which preserve $\mathcal{H}^{(p)}$. The final constrained system is the restriction of this algebra to the subspace $\mathcal{H}^{(p)}$. On abstraction of such a system into $\mathrm{C}^{*}$-algebra terms, one starts with a unital $\mathrm{C}^{*}$-algebra $\mathcal{F}$ (the field algebra) containing all physical relevant observables. This is an abstract $\mathrm{C}^{*}$-algebra, i.e. we ignore the initial representation in which the system may be defined. We need to decide in what form the constraints should appear in $\mathcal{F}$ as a subset $\mathcal{C}$. We have the following possibilities:

- If all $A_{i}$ are bounded we can identify $\mathcal{C}$ directly with $\left\{A_{i} \mid i \in I\right\} \subset \mathcal{F}$.

- If the $A_{i}$ are unbounded but essentially selfadjoint, we can take $\mathcal{C}:=\{U-\mathbb{1} \mid U \in \mathcal{U}\}=$ : $\mathcal{U}-\mathbb{1}$, where the set of unitaries $\mathcal{U} \subset \mathcal{F}$ is identified with $\left\{\exp \left(i t \bar{A}_{j}\right) \mid t \in \mathbb{R}, j \in I\right\}$. This is the form in which constraints were analyzed in [3], and also the form which we will use here in the following sections.

- If the $A_{i}$ are unbounded and normal, we can identify $\mathcal{C}$ with $\left\{f\left(A_{j}\right) \mid j \in I\right\}$ where $f$ is a bounded real valued Borel function with $f^{-1}(0)=\{0\}$.

- If the $A_{i}$ are unbounded, closable and not normal, then we can replace each $A_{i}$ by the essentially selfadjoint operator $A_{i}^{*} A_{i}$ which is justified because for any closed operator $A$ we have $\operatorname{Ker} A=\operatorname{Ker} A^{*} A$, reducing this case to the one for essentially selfadjoint constraints.

Finally, notice that we can replace any constraint set $\mathcal{C}$ as above, by one which satisfies $\mathcal{C}^{*}=\mathcal{C}$ as a set and which selects the same physical subspace, using the fact that $\operatorname{Ker} A=\operatorname{Ker} A^{*} A$.

Motivated from above, our starting point is:

2.1 Definition $A$ quantum system with constraints is a pair $(\mathcal{F}, \mathcal{C})$ where the field algebra $\mathcal{F}$ is a unital $\mathrm{C}^{*}$-algebra containing the constraint set $\mathcal{C}=\mathcal{C}^{*}$. A constraint condition on $(\mathcal{F}, \mathcal{C})$ consists of the selection of the physical state space by:

$$
\mathfrak{S}_{D}:=\left\{\omega \in \mathfrak{S}(\mathcal{F}) \mid \pi_{\omega}(C) \Omega_{\omega}=0 \quad \forall C \in \mathcal{C}\right\},
$$

where $\mathfrak{S}(\mathcal{F})$ denotes the state space of $\mathcal{F}$, and $\left(\pi_{\omega}, \mathcal{H}_{\omega}, \Omega_{\omega}\right)$ denotes the GNS-data of $\omega$. The elements of $\mathfrak{S}_{D}$ are called Dirac states. The case of unitary constraints means that $\mathcal{C}=\mathcal{U}-\mathbb{1}$, $\mathcal{U} \subset \mathcal{F}_{u}$, and for this we will also use the notation $(\mathcal{F}, \mathcal{U})$. 
Thus in the GNS-representation of each Dirac state, the GNS cyclic vector $\Omega_{\omega}$ satisfies the physical selection condition above. The assumption is that all physical information is contained in the pair $\left(\mathcal{F}, \mathfrak{S}_{D}\right)$.

For the case of unitary constraints we have the following equivalent characterizations of the Dirac states (cf. [3, Theorem 2.19 (ii)]):

$$
\begin{aligned}
\mathfrak{S}_{D} & =\{\omega \in \mathfrak{S}(\mathcal{F}) \mid \omega(U)=1 \quad \forall U \in \mathcal{U}\} \\
& =\{\omega \in \mathfrak{S}(\mathcal{F}) \mid \omega(F U)=\omega(F)=\omega(U F) \quad \forall F \in \mathcal{F}, U \in \mathcal{U}\} .
\end{aligned}
$$

Moreover, the set $\left\{\alpha_{U}:=\operatorname{Ad}(U) \mid U \in \mathcal{U}\right\}$ of automorphisms of $\mathcal{F}$ leaves every Dirac state invariant, i.e. we have $\omega \circ \alpha_{U}=\omega$ for all $\omega \in \mathfrak{S}_{D}, U \in \mathcal{U}$.

For a general constraint set $\mathcal{C}$, observe that we have:

$$
\begin{aligned}
\mathfrak{S}_{D} & =\left\{\omega \in \mathfrak{S}(\mathcal{F}) \mid \omega\left(C^{*} C\right)=0 \quad \forall C \in \mathcal{C}\right\} \\
& =\left\{\omega \in \mathfrak{S}(\mathcal{F}) \mid \mathcal{C} \subseteq N_{\omega}\right\}=\mathcal{N}^{\perp} \cap \mathfrak{S}(\mathcal{F}) .
\end{aligned}
$$

Here $N_{\omega}:=\left\{F \in \mathcal{F} \mid \omega\left(F^{*} F\right)=0\right\}$ is the left kernel of $\omega$ and $\mathcal{N}:=\cap\left\{N_{\omega} \mid \omega \in \mathfrak{S}_{D}\right\}$, and the superscript $\perp$ denotes the annihilator of the corresponding subset in the dual of $\mathcal{F}$. The equality $\mathcal{N}=[\mathcal{F C}]$ (where we use the notation [.] for the closed linear space generated by its argument), follows from the fact that every closed left ideal is the intersection of the left kernels which contains it (cf. 3.13 .5 in [9]). Thus $\mathcal{N}$ is the left ideal generated by $\mathcal{C}$. Since $\mathcal{C}$ is selfadjoint and contained in $\mathcal{N}$ we conclude $\mathcal{C} \subset \mathrm{C}^{*}(\mathcal{C}) \subset \mathcal{N} \cap \mathcal{N}^{*}=[\mathcal{F C}] \cap[\mathcal{C F}]$, where $\mathrm{C}^{*}(\cdot)$ denotes the $\mathrm{C}^{*}$-algebra in $\mathcal{F}$ generated by its argument.

2.2 Theorem Now for the Dirac states we have:

(i) $\mathfrak{S}_{D} \neq \emptyset$ iff $\mathbb{1} \notin \mathrm{C}^{*}(\mathcal{C})$ iff $\mathbb{1} \notin \mathcal{N} \cap \mathcal{N}^{*}=: \mathcal{D}$.

(ii) $\omega \in \mathfrak{S}_{D}$ iff $\pi_{\omega}(\mathcal{D}) \Omega_{\omega}=0$.

(iii) An extreme Dirac state is pure.

Proof: (i) The first equivalence is proven in Theorem 2.7 of [3]. If $\mathbb{1} \in \mathcal{D} \subset \mathcal{N}$, then $\omega(\mathcal{N}) \neq 0$ for all states $\omega$, i.e. $\mathfrak{S}_{D}=\emptyset$. If $\mathbb{1} \notin \mathcal{D}$, then $\mathbb{1} \notin \mathcal{N}$ so $\mathcal{N}$ is a proper closed left ideal and hence by 3.10 .7 in $[9] \mathfrak{S}_{D} \neq \emptyset$.

(ii) If $\omega \in \mathfrak{S}_{D}$, then $\mathcal{D} \subset \mathcal{N} \subset N_{\omega}$, hence $\pi_{\omega}(\mathcal{D}) \Omega_{\omega}=0$. Conversely, since $\mathcal{C} \subset \mathcal{D}=[\mathcal{F C}] \cap[\mathcal{C F}]$ we have that $\pi_{\omega}(\mathcal{D}) \Omega_{\omega}=0$ implies $\pi_{\omega}(\mathcal{C}) \Omega_{\omega}=0$ hence $\omega \in \mathfrak{S}_{D}$.

(iii) Denote the quasi-state space of $\mathcal{F}$ by $Q$ [9]. We can write the set of Dirac states as

$$
\mathfrak{S}_{D}=\mathfrak{S}(\mathcal{F}) \cap\{\phi \in Q \mid \phi(L)=0 \quad \forall L \in \mathcal{N}\} .
$$

Since $\mathcal{N}$ is a left ideal, if it is in Ker $\omega$, it must be in $N_{\omega}$. By Theorem 3.10.7 in [9] the set $Q_{0}:=\{\phi \in Q \mid \phi(L)=0 \quad \forall L \in \mathcal{N}\}$ is a weak* closed face in $Q$. Now if we can decompose a Dirac state $\omega$, since it is in $Q_{0}$, so are its components by the facial property of $Q_{0}$. These components are multiples of Dirac states, dominated by $\omega$ so $\omega$ cannot be extreme. Thus extreme Dirac states must be pure.

We will call a constraint set $\mathcal{C}$ first class if $\mathbb{1} \notin \mathrm{C}^{*}(\mathcal{C})$, and this is the nontriviality assumption which we henceforth make [10, Section 3].

Now define

$$
\mathcal{O}:=\{F \in \mathcal{F} \mid[F, D]:=F D-D F \in \mathcal{D} \quad \forall D \in \mathcal{D}\}
$$

Then $\mathcal{O}$ is the $\mathrm{C}^{*}$-algebraic analogue of Dirac's observables (the weak commutant of the constraints) [11]. 
2.3 Theorem With the preceding notation we have:

(i) $\mathcal{D}=\mathcal{N} \cap \mathcal{N}^{*}$ is the unique maximal $\mathrm{C}^{*}$-algebra in $\cap\left\{\operatorname{Ker} \omega \mid \omega \in \mathfrak{S}_{D}\right\}$. Moreover $\mathcal{D}$ is a hereditary $\mathrm{C}^{*}$-subalgebra of $\mathcal{F}$.

(ii) $\mathcal{O}=M_{\mathcal{F}}(\mathcal{D}):=\{F \in \mathcal{F} \mid F D \in \mathcal{D} \ni D F \quad \forall D \in \mathcal{D}\}$, i.e. it is the relative multiplier algebra of $\mathcal{D}$ in $\mathcal{F}$.

(iii) $\mathcal{O}=\{F \in \mathcal{F} \mid[F, \mathcal{C}] \subset \mathcal{D}\}$.

(iv) $\mathcal{D}=[\mathcal{O C}]=[\mathcal{C O}]$.

(v) For the case of unitary constraints, i.e. $\mathcal{C}=\mathcal{U}-\mathbb{1}$, we have $\mathcal{U} \subset \mathcal{O}$ and $\mathcal{O}=$ $\left\{F \in \mathcal{F} \mid \alpha_{U}(F)-F \in \mathcal{D} \quad \forall U \in \mathcal{U}\right\}$ where $\alpha_{U}:=\operatorname{Ad} U$.

Proof: (i) The proof of Theorem 2.13 in [3] is still valid for the current more general constraints. To see that $\mathcal{D}$ is hereditary, use Theorem 3.2.1 in [12] and the fact that $\mathcal{N}=[\mathcal{F C}]$ is a closed left ideal of $\mathcal{F}$.

(ii) Since $\mathcal{D}$ is a two-sided ideal for $M_{\mathcal{F}}(\mathcal{D})$ it is obvious that $M_{\mathcal{F}}(\mathcal{D}) \subset \mathcal{O}$. Conversely, consider $B \in \mathcal{O}$, then for any $D \in \mathcal{D}$, we have $B D=D B+D^{\prime} \in \mathcal{N}$ with $D^{\prime}$ some element of $\mathcal{D}$, where we used $\mathcal{F D}=\mathcal{F}\left(\mathcal{N} \cap \mathcal{N}^{*}\right) \subset \mathcal{N}$. Similarly we see that $D B \in \mathcal{N}^{*}$. But then $\mathcal{N} \ni B D=D B+D^{\prime} \in \mathcal{N}^{*}$, so $B D \in \mathcal{N} \cap \mathcal{N}^{*}=\mathcal{D}$. Likewise $D B \in \mathcal{D}$ and so $B \in M_{\mathcal{F}}(\mathcal{D})$.

(iii) Since $\mathcal{C} \subset \mathcal{D}$ we see from the definition of $\mathcal{O}$ that $F \in \mathcal{O}$ implies that $[F, \mathcal{C}] \subset \mathcal{D}$. Conversely, let $[F, \mathcal{C}] \subset \mathcal{D}$ for some $F \in \mathcal{F}$. Now $F[\mathcal{F C}] \subset[\mathcal{F C}]$ and $F[\mathcal{C F}]=[F \mathcal{C F}] \subset[(\mathcal{C F}+$ $\mathcal{D}) \mathcal{F}] \subset[\mathcal{C F}]$ because $\mathcal{C} F+\mathcal{D} \subset \mathcal{C} F+[\mathcal{C F}] \subset[\mathcal{C F}]$. Thus $F \mathcal{D}=F([\mathcal{F C}] \cap[\mathcal{C F}]) \subset[\mathcal{F C}] \cap[\mathcal{C} \mathcal{F}]=\mathcal{D}$. Similarly $\mathcal{D} F \subset \mathcal{D}$, and thus by (ii) we see $F \in \mathcal{O}$.

(iv) $\mathcal{D} \subset \mathcal{O}$ so by (i) it is the unique maximal $\mathrm{C}^{*}$-algebra annihilated by all the states $\omega \in$ $\mathfrak{S}_{D}(\mathcal{O})=\mathfrak{S}_{D}\lceil\mathcal{O}$ (since $\mathcal{C} \subset \mathcal{O}$ ). Thus $\mathcal{D}=[\mathcal{O C}] \cap[\mathcal{C O}]$. But $\mathcal{C} \subset \mathcal{D}$, so by (ii) $[\mathcal{O C}] \subset \mathcal{D} \subset[\mathcal{O C}]$ and so $\mathcal{D}=[\mathcal{O C}]=[\mathcal{C O}]$.

(v) $\mathcal{U} \subset \mathcal{O}$ because $\mathcal{U}-\mathbb{1} \subset \mathcal{D} \subset \mathcal{O} \ni \mathbb{1}$, and so $[F, \mathcal{C}] \subset \mathcal{D}$ implies $[U-\mathbb{1}, F] U^{-1}=$ $\alpha_{U}(F)-F \in \mathcal{D}$ for $U \in \mathcal{U}$. The converse is similar.

Thus $\mathcal{D}$ is a closed two-sided ideal of $\mathcal{O}$ and it is proper when $\mathfrak{S}_{D} \neq \emptyset$ (which we assume here by $\mathbb{1} \notin \mathrm{C}^{*}(\mathcal{C})$ ). From (iii) above, we see that the traditional observables $\mathcal{C}^{\prime} \subset \mathcal{O}$, where $\mathcal{C}^{\prime}$ denotes the relative commutant of $\mathcal{C}$ in $\mathcal{F}$.

Define the maximal $\mathrm{C}^{*}$-algebra of physical observables as

$$
\mathcal{R}:=\mathcal{O} / \mathcal{D}
$$

The factoring procedure is the actual step of imposing constraints. This method of constructing $\mathcal{R}$ from $(\mathcal{F}, \mathcal{C})$ is called the $\mathbf{T}$-procedure in $[13]$. We require that after the $\mathrm{T}$-procedure all physical information is contained in the pair $(\mathcal{R}, \mathfrak{S}(\mathcal{R}))$, where $\mathfrak{S}(\mathcal{R})$ denotes the set of states on $\mathcal{R}$. Now, it is possible that $\mathcal{R}$ may not be simple [13], Section 2], and this would not be acceptable for a physical algebra. So, using physical arguments, one would in practice choose a $\mathrm{C}^{*}$-subalgebra $\mathcal{O}_{c} \subset \mathcal{O}$ containing the traditional observables $\mathcal{C}^{\prime}$ such that

$$
\mathcal{R}_{c}:=\mathcal{O}_{c} /\left(\mathcal{D} \cap \mathcal{O}_{c}\right) \subset \mathcal{R},
$$

is simple. The following result justifies the choice of $\mathcal{R}$ as the algebra of physical observables (cf. Theorem 2.20 in [3]):

2.4 Theorem There exists a $w^{*}$-continuous isometric bijection between the Dirac states on $\mathcal{O}$ and the states on $\mathcal{R}$.

The hereditary property of $\mathcal{D}$ can be further analyzed, and we do this in Appendix 1 (it will be useful occasionally in proofs). 


\section{Local Quantum Constraints}

In this section we will introduce our main object of study, viz a system of local quantum constraints. In practice, a large class of constraint systems occur in quantum field theory (henceforth denoted by QFT), for instance gauge theories. A prominent property of a QFT, is space-time locality, and usually when constraints occur, they also have this property. Heuristically such constraints are written as

$$
\chi(x) \psi=0 \quad \text { for } \quad \psi \in \mathcal{H}^{(p)}, x \in M^{4},
$$

where Minkowski space is $M^{4}=\left(\mathbb{R}^{4}, \eta\right)$ with metric $\eta:=\operatorname{diag}(+,-,-,-)$, and this makes the locality explicit. Since $\chi$ is actually an operator-valued distribution, the correct expression should be of the form

$$
\chi(f) \psi=0 \quad \text { for } \quad \psi \in \mathcal{H}^{(p)}, f \in C_{c}^{\infty}\left(\mathbb{R}^{4}\right) .
$$

In this section we now want to analyze how locality intertwines with the $\mathrm{T}$-procedure of constraint reduction. To make this precise, recall that the Haag-Kastler axioms [1], 2] express locality for a QFT as follows:

3.1 Definition A Haag-Kastler QFT (or HK-QFT for short) consists of the following.

- $A$ directed set $\Gamma \subseteq\left\{\Theta \subset M^{4} \mid \Theta\right.$ open and bounded $\}$ partially ordered by set inclusion, such that $\mathbb{R}^{4}=\cup\{\Theta \mid \Theta \in \Gamma\}$ and under the action of the orthochronous Poincaré group $\mathcal{P}_{+}^{\uparrow}$ on $M^{4}$ we have $g \Theta \in \Gamma$ for all $\Theta \in \Gamma, g \in \mathcal{P}_{+}^{\uparrow}$.

- $A$ directed set $\widetilde{\Gamma}$ of $\mathrm{C}^{*}$-algebras with a common identity $\mathbb{1}$, ordered by inclusion, with an inductive limit $\mathrm{C}^{*}$-algebra $\mathcal{F}_{0}$ (over $\widetilde{\Gamma}$ ). We will call the elements of $\widetilde{\Gamma}$ the local field algebras and $\mathcal{F}_{0}$ the quasi-local algebra.

- A surjection $\mathcal{F}: \Gamma \rightarrow \widetilde{\Gamma}$, satisfying:

(1) (Isotony) $\mathcal{F}$ is order preserving, i.e. $\mathcal{F}\left(\Theta_{1}\right) \subseteq \mathcal{F}\left(\Theta_{2}\right)$ if $\Theta_{1} \subseteq \Theta_{2}$.

(2) (Causality) If $\Theta_{1}, \Theta_{2} \in \Gamma$ are spacelike separated, (henceforth denoted $\Theta_{1} \perp \Theta_{2}$ ), then $\left[\mathcal{F}\left(\Theta_{1}\right), \mathcal{F}\left(\Theta_{2}\right)\right]=0$ in $\mathcal{F}_{0}$.

(3) (Covariance) There is an action $\alpha: \mathcal{P}_{+}^{\uparrow} \rightarrow$ Aut $\mathcal{F}_{0}$ such that $\alpha_{g}(\mathcal{F}(\Theta))=\mathcal{F}(g \Theta)$, $g \in \mathcal{P}_{+}^{\uparrow}, \Theta \in \Gamma$.

3.2 Remark (i) In the usual algebraic approach to QFT (cf. [2]), there are additional axioms, e.g. that $\mathcal{F}_{0}$ must be primitive, that there is a vacuum state with GNS-representation in which the generators of the translations in the covariant representation of $\mathcal{P}_{+}^{\uparrow}$ have spectra in the forward light cone, that there is a compact gauge group, local definiteness, local normality etc. We will return to some of these axioms later, but for now, we restrict our analysis to those listed in Definition 3.1. The net $\Gamma$ is usually taken to be the set of double cones in $M^{4}$, but here we will keep it more general. There is some redundancy in this definition e.g. for the results in this Section, we will not need the assumption that $\Theta \in \Gamma$ is bounded.

(ii) In the literature, sometimes "local" is used synonymously with "causal." Since we want to weaken the Haag-Kastler axioms below, we will make a distinction between these terms, in particular, we will call an algebra $\mathcal{F}(\Theta)$ in an isotone net as above local, (as well as its elements). If such an isotone net also satisfies causality, it is called causal. 
In the context of the Haag-Kastler axioms, we would like to define a system of "local quantum constraints" in such a way that it includes the major examples from QFT.

3.3 Definition $A$ system of local quantum constraints consists of a surjection $\mathcal{F}: \Gamma \rightarrow \widetilde{\Gamma}$ as in Definition 3.1 satisfying isotony as well as

(4) (Local Constraints) There is a map $\mathcal{U}$ from $\Gamma$ to the set of first class subsets of the unitaries in the local field algebras such that

$$
\begin{aligned}
& \mathcal{U}(\Theta) \subset \mathcal{F}(\Theta)_{u} \text { for all } \Theta \in \Gamma, \text { and } \\
& \text { if } \Theta_{1} \subseteq \Theta_{2} \text {, then } \mathcal{U}\left(\Theta_{1}\right)=\mathcal{U}\left(\Theta_{2}\right) \cap \mathcal{F}\left(\Theta_{1}\right) .
\end{aligned}
$$

3.4 Remark In this definition we have made the minimum assumptions to start the analysis. We have omitted causality and covariance because these are physical requirements which one should demand for the final physical theory, not the initial (unconstrained) theory which contains nonphysical objects. There are examples of constrained QFTs satisfying these conditions, e.g. [14, Remark 4.3], [15, Chapter 4] and see also below in Section 5 our example of Gupta-Bleuler electromagnetism.

Given a system of local quantum constraints, $\Theta \rightarrow(\mathcal{F}(\Theta), \mathcal{U}(\Theta))$, we can apply the Tprocedure to each local system $(\mathcal{F}(\Theta), \mathcal{U}(\Theta))$, to obtain the "local" objects:

$$
\begin{aligned}
\mathfrak{S}_{D}^{\Theta} & :=\{\omega \in \mathfrak{S}(\mathcal{F}(\Theta)) \mid \omega(U)=1 \quad \forall U \in \mathcal{U}(\Theta)\}=\mathfrak{S}_{D}(\mathcal{F}(\Theta)), \\
\mathcal{D}(\Theta) & :=[\mathcal{F}(\Theta) \mathcal{C}(\Theta)] \cap[\mathcal{C}(\Theta) \mathcal{F}(\Theta)], \\
\mathcal{O}(\Theta) & :=\{F \in \mathcal{F}(\Theta) \mid F D-D F \in \mathcal{D}(\Theta) \quad \forall D \in \mathcal{D}(\Theta)\}=M_{\mathcal{F}(\Theta)}(\mathcal{D}(\Theta)), \\
\mathcal{R}(\Theta) & :=\mathcal{O}(\Theta) / \mathcal{D}(\Theta) .
\end{aligned}
$$

We can be now more precise about what our task is in this section:

Problem: For a system of local quantum constraints $\Theta \rightarrow(\mathcal{F}(\Theta), \mathcal{U}(\Theta))$, find minimal conditions such that the net of local physical observables $\Theta \rightarrow \mathcal{R}(\Theta)$ becomes a HK-QFT.

Now, to analyze the isotony property, we need to determine what the inclusions in Definition 3.3 imply for the associated objects $\left(\mathfrak{S}_{D}^{\Theta_{i}}, \mathcal{D}\left(\Theta_{i}\right), \mathcal{O}\left(\Theta_{i}\right), \mathcal{F}\left(\Theta_{i}\right)\right)$, and this is the task of the next subsection.

\subsection{Inclusion structures}

For this subsection we assume unitary first class constraints $\mathcal{C}=\mathcal{U}-\mathbb{1}$, indicated by a pair $(\mathcal{F}, \mathcal{U})$. Motivated by Definition 3.3, we define:

3.5 Definition $A$ first class constrained system $(\mathcal{F}, \mathcal{U})$ is said to be included in another one $\left(\mathcal{F}_{e}, \mathcal{U}_{e}\right)$, if the $\mathrm{C}^{*}$-algebras $\mathcal{F} \subset \mathcal{F}_{e}$ have a common identity and $\mathcal{U}=\mathcal{U}_{e} \cap \mathcal{F}$. We denote this by $(\mathcal{F}, \mathcal{U}) \subseteq\left(\mathcal{F}_{e}, \mathcal{U}_{e}\right)$

For the rest of this subsection we will assume that $(\mathcal{F}, \mathcal{U}) \subseteq\left(\mathcal{F}_{e}, \mathcal{U}_{e}\right)$. From the T-procedure sketched above we obtain the corresponding quadruples

$$
\left(\mathfrak{S}_{D}, \mathcal{D}, \mathcal{O}, \mathcal{R}\right) \quad \text { and } \quad\left(\mathfrak{S}_{D e}, \mathcal{D}_{e}, \mathcal{O}_{e}, \mathcal{R}_{e}\right)
$$


3.6 Lemma Suppose that $\mathcal{D}=\mathcal{D}_{e} \cap \mathcal{O}$ and $\mathcal{O} \subset \mathcal{O}_{e}$, then there is a ${ }^{*}$-isomorphism from $\mathcal{R}$ to a $\mathrm{C}^{*}$-subalgebra of $\mathcal{R}_{e}$, which maps the identity of $\mathcal{R}$ to the identity of $\mathcal{R}_{e}$.

Proof: From

$$
\mathcal{R}_{e}=\mathcal{O}_{e} / \mathcal{D}_{e}=\left(\mathcal{O} / \mathcal{D}_{e}\right) \cup\left(\left(\mathcal{O}_{e} \backslash \mathcal{O}\right) / \mathcal{D}_{e}\right),
$$

it is enough to show that $\mathcal{O} / \mathcal{D}_{e} \cong \mathcal{R}=\mathcal{O} / \mathcal{D}$. Now, a $\mathcal{D}_{e}$-equivalence class consists of $A, B \in \mathcal{O}$ such that $A-B \in \mathcal{D}_{e}$ and therefore $A-B \in \mathcal{D}_{e} \cap \mathcal{O}=\mathcal{D}$. This implies $\mathcal{O} / \mathcal{D}_{e} \cong \mathcal{O} / \mathcal{D}=\mathcal{R}$. Moreover, since $\mathbb{1} \in \mathcal{O} \subset \mathcal{O}_{e}$, and the $\mathcal{D}$-equivalence class of $\mathbb{1}$ is contained in the $\mathcal{D}_{e}$-equivalence class of $\mathbb{1}$, it follows that the identity maps to the identity.

3.7 Remark (i) By a simple finite dimensional example one can verify that the condition $\mathcal{D}=\mathcal{D}_{e} \cap \mathcal{O}$ does not imply $\mathcal{O} \subset \mathcal{O}_{e}$.

(ii) Instead of the conditions in Lemma 3.6 another natural set of restriction conditions one can also choose is $\mathcal{D}=\mathcal{D}_{e} \cap \mathcal{F}$ and $\mathcal{O}=\mathcal{O}_{e} \cap \mathcal{F}$, but below we will see that these imply those in Lemma 3.6.

The next result gives sufficient conditions for the equation $\mathcal{D}=\mathcal{D}_{e} \cap \mathcal{O}$ to hold.

3.8 Lemma $\mathcal{D}=\mathcal{D}_{e} \cap \mathcal{F}$ if either

(i) $\mathfrak{S}_{D}=\mathfrak{S}_{D e} \uparrow \mathcal{F} \quad$ or

(ii) $\left[\mathcal{F} \mathrm{C}^{*}(\mathcal{U}-\mathbb{1})\right]=\left[\mathcal{F}_{e} \mathrm{C}^{*}\left(\mathcal{U}_{e}-\mathbb{1}\right)\right] \cap \mathcal{F}$.

Proof: Take $\omega \in \mathfrak{S}_{D e}$ and recall the definition for the left kernel $N_{\omega}$ given in the preceding subsection. Then $N_{\omega} \cap \mathcal{F}=N_{\omega \mid \mathcal{F}}$ and from (i) we get

$$
\begin{aligned}
\mathcal{N}=\cap\left\{N_{\omega} \mid \omega \in \mathfrak{S}_{D}\right\} & =\cap\left\{N_{\omega}\left|\omega \in \mathfrak{S}_{D e}\right| \mathcal{F}\right\} \\
& =\cap\left\{N_{\omega} \cap \mathcal{F} \mid \omega \in \mathfrak{S}_{D e}\right\} \\
& =\cap\left\{N_{\omega} \mid \omega \in \mathfrak{S}_{D e}\right\} \cap \mathcal{F}=\mathcal{N}_{e} \cap \mathcal{F}
\end{aligned}
$$

This produces

$$
\mathcal{N}=\left[\mathcal{F} \mathrm{C}^{*}(\mathcal{U}-\mathbb{1})\right]=\left[\mathcal{F}_{e} \mathrm{C}^{*}\left(\mathcal{U}_{e}-\mathbb{1}\right)\right] \cap \mathcal{F}
$$

as well as

$$
\begin{aligned}
\mathcal{D} & =\left[\mathcal{F} \mathrm{C}^{*}(\mathcal{U}-\mathbb{1})\right] \cap\left[\mathrm{C}^{*}(\mathcal{U}-\mathbb{1}) \mathcal{F}\right] \\
& =\left[\mathcal{F}_{e} \mathrm{C}^{*}\left(\mathcal{U}_{e}-\mathbb{1}\right)\right] \cap\left[\mathrm{C}^{*}\left(\mathcal{U}_{e}-\mathbb{1}\right) \mathcal{F}_{e}\right] \cap \mathcal{F} \\
& =\mathcal{D}_{e} \cap \mathcal{F}
\end{aligned}
$$

and the proof is concluded.

3.9 Lemma We have

$$
\mathcal{O} \subset \mathcal{O}_{e} \quad \text { iff } \quad \mathcal{O} \subset\left\{F \in \mathcal{F} \mid U F U^{-1}-F \in \mathcal{D}_{e} \quad \forall U \in\left(\mathcal{U}_{e} \backslash \mathcal{U}\right)\right\}
$$


Proof: By Theorem 2.3 (v) we have

$$
\begin{aligned}
& \mathcal{O}_{e} \cap \mathcal{F}=\left\{F \in \mathcal{F} \mid U F U^{-1}-F \in \mathcal{D}_{e} \quad \forall U \in \mathcal{U}_{e}\right\} \\
&=\left\{F \in \mathcal{F} \mid U F U^{-1}-F \in \mathcal{D}_{e} \cap \mathcal{F} \quad \forall U \in \mathcal{U}\right\} \\
& \cap\left\{F \in \mathcal{F} \mid U F U^{-1}-F \in \mathcal{D}_{e} \quad \forall U \in\left(\mathcal{U}_{e} \backslash \mathcal{U}\right)\right\} \\
&=\left(\mathcal{O} \cup\left\{F \in \mathcal{F} \mid U F U^{-1}-F \in\left(\mathcal{D}_{e} \cap \mathcal{F} \backslash \mathcal{D}\right) \quad \forall U \in \mathcal{U}\right\}\right) \\
& \cap\left\{F \in \mathcal{F} \mid U F U^{-1}-F \in \mathcal{D}_{e} \quad \forall U \in\left(\mathcal{U}_{e} \backslash \mathcal{U}\right)\right\} \\
&=\left(\mathcal{O} \cap\left\{F \in \mathcal{F} \mid U F U^{-1}-F \in \mathcal{D}_{e} \quad \forall U \in\left(\mathcal{U}_{e} \backslash \mathcal{U}\right)\right\}\right) \\
& \cup\left\{F \in \mathcal{F} \mid U F U^{-1}-F \in\left(\mathcal{D}_{e} \cap \mathcal{F} \backslash \mathcal{D}\right) \quad \forall U \in \mathcal{U}\right. \\
&\text { and } \left.U F U^{-1}-F \in \mathcal{D}_{e} \quad \forall U \in\left(\mathcal{U}_{e} \backslash \mathcal{U}\right)\right\}
\end{aligned}
$$

Now if $\mathcal{O} \subset\left\{F \in \mathcal{F} \mid U F U^{-1}-F \in \mathcal{D}_{e} \quad \forall U \in\left(\mathcal{U}_{e} \backslash \mathcal{U}\right)\right\}$, then it is clear from the above equations that $\mathcal{O} \subset \mathcal{O}_{e} \cap \mathcal{F} \subset \mathcal{O}_{e}$.

To prove the other implication note that the second set in the union of Eqn. (3) is contained in $\mathcal{F} \backslash \mathcal{O}$ and therefore from $\mathcal{O} \subset \mathcal{O}_{e}$ we obtain

$$
\mathcal{O}=\mathcal{O} \cap \mathcal{O}_{e} \cap \mathcal{F}=\mathcal{O} \cap\left\{F \in \mathcal{F} \mid U F U^{-1}-F \in \mathcal{D}_{e} \quad \forall U \in\left(\mathcal{U}_{e} \backslash \mathcal{U}\right)\right\}
$$

which implies the desired inclusion.

3.10 Theorem Given an included pair of first class constrained systems $(\mathcal{F}, \mathcal{U}) \subseteq\left(\mathcal{F}_{e}, \mathcal{U}_{e}\right)$ and notation as above, the following statements are in the relation $(\mathrm{iv}) \Rightarrow($ iii $) \Rightarrow($ ii $) \Rightarrow(i)$ where:

(i) $\mathfrak{S}_{D} \uparrow \mathcal{O}=\mathfrak{S}_{D e} \uparrow \mathcal{O}$.

(ii) $\mathcal{D}=\mathcal{D}_{e} \cap \mathcal{O}$ and $\mathcal{O} \subseteq \mathcal{O}_{e}$.

(iii) $\mathcal{D}=\mathcal{D}_{e} \cap \mathcal{F}$ and $\mathcal{O}=\mathcal{O}_{e} \cap \mathcal{F}$.

(iv) $\mathfrak{S}_{D}=\mathfrak{S}_{D e} \uparrow \mathcal{F}$ and $\mathcal{O} \subseteq \mathcal{O}_{e}$

Proof: We first prove the implication $(i i) \Rightarrow(i)$, so assume $(i i)$. It suffices to show that all Dirac states on $\mathcal{O}$ extend to Dirac states on $\mathcal{O}_{e}$. Denote by $\mathfrak{S}(\mathcal{R})$ and $\mathfrak{S}_{D}(\mathcal{O})$ respectively the set of states on $\mathcal{R}$ and the set of Dirac states on $\mathcal{O}$ (assume also corresponding notation for $\mathcal{R}_{e}$ and $\left.\mathcal{O}_{e}\right)$. Then from Theorem 2.4 there exist $w^{*}$-continuous, isometric bijections $\theta$ and $\theta_{e}$ :

$$
\theta: \mathfrak{S}_{D}(\mathcal{O}) \rightarrow \mathfrak{S}(\mathcal{R}) \quad \text { and } \quad \theta_{e}: \mathfrak{S}_{D e}\left(\mathcal{O}_{e}\right) \rightarrow \mathfrak{S}\left(\mathcal{R}_{e}\right)
$$

Now take $\omega \in \mathfrak{S}_{D}(\mathcal{O})$, so that $\theta(\omega) \in \mathfrak{S}(\mathcal{R})$. From Lemma 3.6, $\mathcal{R} \subset \mathcal{R}_{e}$ so we can extend $\theta(\omega)$ to $\widetilde{\theta(\omega)} \in \mathfrak{S}\left(\mathcal{R}_{e}\right)$. Finally, $\theta_{e}^{-1}(\widetilde{\theta(\omega)}) \in \mathfrak{S}_{D e}\left(\mathcal{O}_{e}\right)$ is an extension of $\omega$, since for any $A \in \mathcal{O}$ we have

$$
\theta_{e}^{-1}(\widetilde{\theta(\omega)})(A)=\widetilde{\theta(\omega)}\left(\xi_{e}(A)\right)=\theta(\omega)(\xi(A))=\omega(A)
$$

where $\xi: \mathcal{O} \rightarrow \mathcal{R}$ and $\xi_{e}: \mathcal{O}_{e} \rightarrow \mathcal{R}_{e}$ are the canonical factorization maps. This proves $(i)$.

Next we prove $($ iii $) \Rightarrow($ ii $)$. Obviously $\mathcal{O}=\mathcal{O}_{e} \cap \mathcal{F}$ implies that $\mathcal{O} \subseteq \mathcal{O}_{e}$. Since $\mathcal{D} \subset \mathcal{O} \subset \mathcal{F}$ we have $\mathcal{D}=\mathcal{D}_{e} \cap \mathcal{F} \subset \mathcal{O}$ and so $\mathcal{D}=\left(\mathcal{D}_{e} \cap \mathcal{F}\right) \cap \mathcal{O}=\mathcal{D}_{e} \cap \mathcal{O}$. 
Finally we prove $(i v) \Rightarrow(i i i)$. By Lemma $3.8(\mathrm{i}), \mathfrak{S}_{D}=\mathfrak{S}_{D e} \uparrow \mathcal{F}$ implies that $\mathcal{D}=\mathcal{D}_{e} \cap \mathcal{F}$. By Theorem 2.3(iii) and the fact that $\mathcal{O}$ is a $\mathrm{C}^{*}$-algebra (hence the span of its selfadjoint elements), we have

$$
\begin{aligned}
\mathcal{O} & =\left[\left\{F \in \mathcal{F}_{s a} \mid[F, \mathcal{U}] \subset \mathcal{D}\right\}\right] \\
& =\left[\left\{F \in \mathcal{F}_{s a} \mid \omega\left(\left[F, U^{*}\right] \cdot[F, U]\right)=0 \quad \forall \omega \in \mathfrak{S}_{D}, U \in \mathcal{U}\right\}\right]
\end{aligned}
$$

where the last equality follows from $\mathcal{D}=\mathcal{N} \cap \mathcal{N}^{*},[F, U]^{*}=-\left[F, U^{*}\right]$ for $F=F^{*}$, and $\mathcal{U}=\mathcal{U}^{*}$. Since $\mathfrak{S}_{D}=\mathfrak{S}_{D e} \mid \mathcal{F}$ we have

$$
\begin{aligned}
\mathcal{O} & =\left[\left\{F \in \mathcal{F}_{s a} \mid \omega\left(\left[F, U^{*}\right] \cdot[F, U]\right)=0 \quad \forall \omega \in \mathfrak{S}_{D e}, U \in \mathcal{U}\right\}\right] \\
& \supseteq\left[\left\{F \in \mathcal{F}_{s a} \mid \omega\left(\left[F, U^{*}\right] \cdot[F, U]\right)=0 \quad \forall \omega \in \mathfrak{S}_{D e}, U \in \mathcal{U}_{e}\right\}\right] \\
& =\mathcal{O}_{e} \cap \mathcal{F} .
\end{aligned}
$$

Thus since $\mathcal{O} \subseteq \mathcal{O}_{e}$ we conclude that $\mathcal{O}=\mathcal{O}_{e} \cap \mathcal{F}$.

3.11 Remark $\mathfrak{S}_{D}\left|\mathcal{O}=\mathfrak{S}_{D e}\right| \mathcal{O}$ does not seem to imply that $\mathfrak{S}_{D}=\mathfrak{S}_{D e} \mid \mathcal{F}$ since for a state in $\mathfrak{S}_{D}$, if one restricts it to $\mathcal{O}$ and then extends to a state in $\mathfrak{S}_{D e}$, it seems nontrivial whether this extension can coincide with the original state on $\mathcal{F}$.

\subsection{Isotony and causality weakened.}

Return now to the previous analysis of a system of local quantum constraints, and define:

3.12 Definition Fix a system of local quantum constraints $\Theta \rightarrow(\mathcal{F}(\Theta), \mathcal{U}(\Theta)$ ) (cf. Def. 3.马), then we say that it satisfies:

(5) reduction isotony if $\Theta_{1} \subseteq \Theta_{2}$ implies $\mathcal{O}\left(\Theta_{1}\right) \subseteq \mathcal{O}\left(\Theta_{2}\right)$ and $\mathcal{D}\left(\Theta_{1}\right)=\mathcal{D}\left(\Theta_{2}\right) \cap \mathcal{O}\left(\Theta_{1}\right)$ (cf. Lemma 3.6 for motivation).

(6) weak causality if for $\Theta_{1} \perp \Theta_{2}$ there is some $\Theta_{0} \supset \Theta_{1} \cup \Theta_{2}, \Theta_{0} \in \Gamma$ such that

$$
\left[\mathcal{O}\left(\Theta_{1}\right), \mathcal{O}\left(\Theta_{2}\right)\right] \subset \mathcal{D}\left(\Theta_{0}\right)
$$

3.13 Remark (i) Given a system with reduction isotony, we have by Lemma 3.6 that when $\Theta_{1} \subseteq \Theta_{2}$, then $\mathcal{R}\left(\Theta_{1}\right)$ is isomorphic to a $\mathrm{C}^{*}$-subalgebra of $\mathcal{R}\left(\Theta_{2}\right)$, which we will denote as $\iota_{12}: \mathcal{R}\left(\Theta_{1}\right) \rightarrow \mathcal{R}\left(\Theta_{2}\right)$.

(ii) The weak causality condition is considerably weaker than requiring causality (cf. (2) in Definition 3.1) for the field algebra, and this will be crucial below for Gupta-Bleuler electromagnetism.

Now we state our first major claim.

3.14 Theorem Let $\Gamma \ni \Theta \rightarrow(\mathcal{F}(\Theta), \mathcal{U}(\Theta))$ be a system of local quantum constraints.

(i) If it satisfies reduction isotony, then $\Theta \rightarrow \mathcal{R}(\Theta)$ has isotony, i.e. $\Theta_{1} \subset \Theta_{2}$, implies $\mathcal{R}\left(\Theta_{1}\right) \subset \mathcal{R}\left(\Theta_{2}\right)$. In this case, the net $\Theta \rightarrow \mathcal{R}(\Theta)$ has an inductive limit, which we denote by $\mathcal{R}_{0}:=\lim _{\longrightarrow} \mathcal{R}(\Theta)$, and call it the quasi-local physical algebra. 
(ii) If it satisfies weak causality and reduction isotony, then $\Theta \rightarrow \mathcal{R}(\Theta)$ has causality, i.e. $\Theta_{1} \perp \Theta_{2}$, implies $\left[\mathcal{R}\left(\Theta_{1}\right), \mathcal{R}\left(\Theta_{2}\right)\right]=0$.

Proof: (i) By reduction isotony we obtain from Lemma 3.6 for $\Theta_{1} \subset \Theta_{2}$ a unital monomorphism $\iota_{12}: \mathcal{R}\left(\Theta_{1}\right) \rightarrow \mathcal{R}\left(\Theta_{2}\right)$. To get isotony from these monomorphisms, we have to verify that they satisfy Takeda's criterion: $\iota_{13}=\iota_{23} \circ \iota_{12}$ (cf. [16]), which will ensure the existence of the inductive limit $\mathcal{R}_{0}$, and in which case we can write simply inclusion $\mathcal{R}\left(\Theta_{1}\right) \subset \mathcal{R}\left(\Theta_{2}\right)$ for $\iota_{12}$. Recall that $\iota_{12}\left(A+\mathcal{D}\left(\Theta_{1}\right)\right)=A+\mathcal{D}\left(\Theta_{2}\right)$ for $A \in \mathcal{O}\left(\Theta_{1}\right)$. Let $\Theta_{1} \subset \Theta_{2} \subset \Theta_{3}$, then by reduction isotony, $\mathcal{O}\left(\Theta_{1}\right) \subset \mathcal{O}\left(\Theta_{2}\right) \subset \mathcal{O}\left(\Theta_{3}\right)$, and so for $A \in \mathcal{O}\left(\Theta_{1}\right), \iota_{23}\left(\iota_{12}\left(A+\mathcal{D}\left(\Theta_{1}\right)\right)\right)=\iota_{23}\left(A+\mathcal{D}\left(\Theta_{2}\right)\right)=$ $A+\mathcal{D}\left(\Theta_{3}\right)=\iota_{13}\left(A+\mathcal{D}\left(\Theta_{1}\right)\right)$. This establishes Takeda's criterion.

(ii) Let $\Theta_{1} \perp \Theta_{2}$ with $\Theta_{0} \supset \Theta_{1} \cup \Theta_{2}$ such that $\left[\mathcal{O}\left(\Theta_{1}\right), \mathcal{O}\left(\Theta_{2}\right)\right] \subset \mathcal{D}\left(\Theta_{0}\right)$ as in Definition 3.12 (6), then since $\mathcal{O}\left(\Theta_{1}\right) \cup \mathcal{O}\left(\Theta_{2}\right) \subset \mathcal{O}\left(\Theta_{0}\right)$ (by reduction isotony), the commutation relation is in $\mathcal{O}\left(\Theta_{0}\right)$, so when we factor out by $\mathcal{D}\left(\Theta_{0}\right)$, the right hand side vanishes and since factoring is a homomorphism, we get $\left[\mathcal{R}\left(\Theta_{1}\right), \mathcal{R}\left(\Theta_{2}\right)\right]=0$ in $\mathcal{R}\left(\Theta_{0}\right)$ and therefore in $\mathcal{R}_{0}$.

Next we would like to analyze the covariance requirement for the net, but here too, we need a preliminary subsection on equivalence of constraints (i.e. when they select the same set of Dirac states), since it will only be necessary for the constraint set to be covariant up to equivalence to ensure that the net of physical algebras is covariant.

\subsection{Equivalent constraints.}

3.15 Definition Two first class constraint sets $\mathcal{C}_{1}, \mathcal{C}_{2}$ for the field algebra $\mathcal{F}$ are called equivalent if they select the same Dirac states, i.e. if for any state $\omega \in \mathfrak{S}(\mathcal{F})$ we have

$$
\mathcal{C}_{1} \subseteq N_{\omega} \quad \text { iff } \quad \mathcal{C}_{2} \subseteq N_{\omega}
$$

In this case we denote $\mathcal{C}_{1} \sim \mathcal{C}_{2}$, and for unitary constraints situation $\mathcal{C}_{i}=\mathcal{U}_{i}-\mathbb{1}, i=1,2$, we also write $\mathcal{U}_{1} \sim \mathcal{U}_{2}$.

3.16 Remark (i) It is clear that the preceding definition introduces an equivalence relation on the family of first class constraint sets for $\mathcal{F}$. Denote by $\mathcal{D}_{i}=\left[\mathcal{F C}_{i}\right] \cap\left[\mathcal{C}_{i} \mathcal{F}\right], i=1,2$, the $\mathrm{C}^{*}$-algebras of Theorem 2.2 (i). Now $\mathcal{C}_{1} \sim \mathcal{C}_{2}$ iff $\mathcal{D}_{1}=\mathcal{D}_{2}$ by Theorems 2.3 (i) and 2.2 (ii), therefore the corresponding observables $\mathcal{O}_{i}$ and physical observables $\mathcal{R}_{i}$ given by the $\mathrm{T}$-procedure will also coincide. This justifies calling these constraint sets equivalent the replacement of $\mathcal{C}_{1}$ by $\mathcal{C}_{2}$ leaves the physics unchanged. Also note that $\mathcal{C} \sim[\mathcal{C}] \sim \mathrm{C}^{*}(\mathcal{C})$. If $\mathcal{C}_{1} \sim \mathcal{C}_{2}$, one can have that $\mathcal{C}_{1}^{\prime} \neq \mathcal{C}_{2}^{\prime}$, i.e. the traditional observables is more sensitive to the choice of constraints than $\mathcal{O}$.

(ii) Whilst the definition of equivalence $\mathcal{C}_{1} \sim \mathcal{C}_{2}$ as stated, depends on $\mathcal{F}$, it depends in fact only on the subalgebra $\mathrm{C}^{*}\left(\mathcal{C}_{1} \cup \mathcal{C}_{2} \cup\{\mathbb{1}\}\right)=: \mathcal{A}$. This is because the extension (resp. restriction) of a Dirac state from (resp. to) a unital $\mathrm{C}^{*}$-subalgebra containing the constraints, is again a Dirac state. Explicitly, the condition: $\omega\left(C^{*} C\right)=0$ for all $C \in \mathcal{C}_{1}$ iff $\omega\left(C^{*} C\right)=0$ for all $C \in \mathcal{C}_{2}$, clearly depends only on the behaviour of $\omega$ on $\mathcal{A}$.

Next we give an algebraic characterization of equivalent constraints, and introduce a maximal constraint set associated to an equivalence class of constraint sets. In the case of unitary constraints, $\mathcal{C}_{i}=\mathcal{U}_{i}-\mathbb{1}$, we obtain a unitary group in $\mathcal{F}$.

3.17 Theorem Let $\mathcal{C}_{i}, i=1,2$, be two first class constraint sets for $\mathcal{F}$, with associated algebras $\mathcal{D}_{i}$ as above. Then 
(i) $\mathcal{C}_{1} \sim \mathcal{C}_{2} \quad$ iff $\quad \mathcal{C}_{1}-\mathcal{C}_{2} \subset \mathcal{D}_{1} \cap \mathcal{D}_{2}$

In the case when $\mathcal{C}_{i}=\mathcal{U}_{i}-\mathbb{1}$, we have $\mathcal{U}_{1} \sim \mathcal{U}_{2} \quad$ iff $\quad \mathcal{U}_{1}-\mathcal{U}_{2} \subset \mathcal{D}_{1} \cap \mathcal{D}_{2}$.

(ii) The maximal constraint set which is equivalent to $\mathcal{C}_{1}$ is $\mathcal{D}_{1}$. In the case when $\mathcal{C}_{1}=\mathcal{U}_{1}-\mathbb{1}$, the set of unitaries

$$
\mathcal{U}_{m}:=\bigcup_{\mathcal{U} \sim \mathcal{U}_{1}} \mathcal{U} \subset \mathcal{F}_{u}
$$

is the maximal set of unitaries equivalent to $\mathcal{U}_{1}$, and it is a group with respect to multiplication in $\mathcal{F}$.

Proof: (i) Suppose that $\mathcal{C}_{1} \sim \mathcal{C}_{2}$ so that by the Remark 3.16 (i), $\mathcal{D}_{1}=\mathcal{D}_{2}$. Then, we have that $\mathcal{C}_{1} \subset \mathcal{D}_{1}=\mathcal{D}_{2} \supset \mathcal{C}_{2}$, and hence $\mathcal{C}_{1}-\mathcal{C}_{2} \subset \mathcal{D}_{1}=\mathcal{D}_{1} \cap \mathcal{D}_{2}$.

Conversely, assume $\mathcal{C}_{1}-\mathcal{C}_{2} \subset \mathcal{D}_{1} \cap \mathcal{D}_{2}$. If $\omega \in \mathfrak{S}(\mathcal{F})$ satisfies $\pi_{\omega}\left(\mathcal{C}_{1}\right) \Omega_{\omega}=0$, then by assumption, $\pi_{\omega}\left(\mathcal{C}_{1}\right) \Omega_{\omega}-\pi_{\omega}\left(\mathcal{C}_{2}\right) \Omega_{\omega} \subseteq \pi_{\omega}\left(\mathcal{D}_{1} \cap \mathcal{D}_{2}\right) \Omega_{\omega} \subset \pi_{\omega}\left(\mathcal{D}_{1}\right) \Omega_{\omega}=0$ using Theorem 2.2 (ii). Thus $\pi_{\omega}\left(\mathcal{C}_{2}\right) \Omega_{\omega}=0$, i.e. $\mathcal{C}_{1} \subset N_{\omega}$ implies that $\mathcal{C}_{2} \subset N_{\omega}$. Interchanging the roles of $\mathcal{C}_{1}$ and $\mathcal{C}_{2}$, we conclude that $\mathcal{C}_{1} \sim \mathcal{C}_{2}$. The second claim for $\mathcal{C}_{i}=\mathcal{U}_{i}-\mathbb{1 1}$ follows from $\mathcal{C}_{1}-\mathcal{C}_{2}=\mathcal{U}_{1}-\mathcal{U}_{2}$.

(ii) That $\mathcal{D}_{1} \sim \mathcal{C}_{1}$ is just the content of Theorem 2.2 (ii). That it is maximal follows from the implication $\mathcal{C}_{2} \sim \mathcal{C}_{1} \Rightarrow \mathcal{C}_{2} \subseteq \mathcal{D}_{2}=\mathcal{D}_{1}$.

For unitary constraints, since $\mathcal{U}_{m}:=\bigcup_{\mathcal{U} \sim \mathcal{U}_{1}} \mathcal{U}$ it follows from part (i) that $\mathcal{U}_{1}-\mathcal{U}_{m} \subset \mathcal{D}_{1}$. Further $\mathcal{U}_{1} \subset \mathcal{U}_{m}$ implies also $\mathcal{D}_{1} \subset \mathcal{D}_{m}$, so that $\mathcal{U}_{1}-\mathcal{U}_{m} \subset \mathcal{D}_{1} \cap \mathcal{D}_{m}=\mathcal{D}_{1}$ and $\mathcal{U}_{m} \sim \mathcal{U}_{1}$. By construction it is also clear that $\mathcal{U}_{m}$ is the maximal unitary constraint set in $\mathcal{F}$ equivalent to $\mathcal{U}_{1}$. We only have to prove that $\mathcal{U}_{m}$ is a group. Let $\mathcal{U}_{0}$ be the group generated in $\mathcal{F}$ by $\mathcal{U}_{m}$. If $\omega \in \mathfrak{S}(\mathcal{F})$ satisfies $\omega\left(\mathcal{U}_{m}\right)=1$, we have $1=\overline{\omega(U)}=\omega\left(U^{*}\right)=\omega\left(U^{-1}\right), U \in \mathcal{U}_{m}$, and also $\omega(U V)=\omega(U)=1, U, V \in \mathcal{U}_{m}$, i.e. $\omega\left(\mathcal{U}_{0}\right)=1$. Thus $\mathcal{U}_{0} \sim \mathcal{U}_{m} \sim \mathcal{U}$ and maximality implies $\mathcal{U}_{0}=\mathcal{U}_{m}$. Hence $\mathcal{U}_{m}$ is a group.

3.18 Remark Observe that for a given unitary constraint system $(\mathcal{F}, \mathcal{U})$ we have that

$$
\mathcal{U}_{m}=\left\{U \in \mathcal{F}_{u} \mid \omega(U)=1 \forall \omega \in \mathfrak{S}_{D}\right\}=\left\{U \in \mathcal{O}_{u} \mid \omega(U)=1 \forall \omega \in \mathfrak{S}_{D}\right\}
$$

since $\mathcal{U}_{m} \subset \mathcal{O}$, cf. Theorem $2.3(\mathrm{v})$.

Next we show that for a large class of first class constraint systems $(\mathcal{F}, \mathcal{C})$ we can find a single constraint in $\mathcal{F}$ which is equivalent to $\mathcal{C}$, and hence can replace it.

3.19 Theorem If $[\mathcal{C}]$ is separable, there exists a positive element $C \in \mathcal{D}_{+}$such that $\{C\} \sim \mathcal{C}$.

Proof: Let $\left\{C_{n}\right\}_{n=1}^{\infty}$ be a denumerable basis of $[\mathcal{C}]$ such that $\left\|C_{n}\right\|<1, n \in \mathbb{N}$. Then

$$
\mathfrak{S}_{D}=\left\{\omega \in \mathfrak{S}(\mathcal{F}) \mid \omega\left(C_{n}^{*} C_{n}\right)=0 \quad \forall n \in \mathbb{N}\right\} .
$$

Define

$$
C:=\sum_{n=1}^{\infty} \frac{C_{n}^{*} C_{n}}{2^{n}} \in \mathcal{D}_{+} .
$$

Then $\omega(C)=0$ iff $\omega\left(C_{n}^{*} C_{n}\right)=0$ for all $n \in \mathbb{N}$, which proves that $\mathfrak{S}_{D}=\{\omega \in \mathfrak{S}(\mathcal{F}) \mid \omega(C)=0\}$. Thus $C^{\frac{1}{2}} \in \mathcal{N}$, but since for any positive operator $A$ we have $\operatorname{Ker} A=\operatorname{Ker} A^{n}$, for all $n \in \mathbb{N}$, we conclude $\mathfrak{S}_{D}=\left\{\omega \in \mathfrak{S}(\mathcal{F}) \mid \omega\left(C^{2}\right)=0\right\}$, so $\{C\} \sim \mathcal{C}$.

3.20 Remark Note that from [12, p. 85] the preceding statement is not true if the separability condition is relaxed. From Remark 8.4(i) we see that if we are willing to enlarge $\mathcal{F}$ to $\mathrm{C}^{*}(\mathcal{F} \cup$ $\{P\})$ for a certain projection $P$, then $\{P\} \sim \mathcal{C}$ so that for this larger algebra, the separability assumption can be omitted. 
3.21 Theorem Let $(\mathcal{F}, \mathcal{C})$ be a first class constraint system, then there is a set of unitaries $\mathcal{U} \subset \mathcal{F}_{u}$ such that $\mathcal{C} \sim \mathcal{U}-\mathbb{1}$ and $\mathcal{U}=\mathcal{U}^{*}$.

Proof: Define the unitaries $\mathcal{U}:=\left\{\exp (i t D) \mid t \in \mathbb{R}, D \in \mathcal{D}_{+}\right\}$. Then for $\omega \in \mathfrak{S}(\mathcal{F})$ we have that $1=\omega(\exp i t D)=1+\sum_{k=1}^{\infty}(i t)^{k} \omega\left(D^{k}\right) / k$ ! for all $t \in \mathbb{R}, D \in \mathcal{D}_{+}$iff $\omega(D)=0$ for all $D \in \mathcal{D}_{+}$iff $\omega(\mathcal{D})=0$ iff $\omega \in \mathfrak{S}_{D}$. Thus $\mathcal{U}-\mathbb{1} \sim \mathcal{C}$. It is obvious that $\mathcal{U}=\mathcal{U}^{*}$.

Hence no constraint system is excluded by the assumption of unitary constraints. Moreover, by Theorem 3.17 there is a canonical unitary group $\mathcal{U}_{m}$ associated with each first class constraint system, and hence a group of inner automorphisms $\operatorname{Ad} \mathcal{U}_{m}$, which one can take as a gauge group in the absence of any further physical restrictions.

\subsection{Weak covariance.}

We define:

3.22 Definition Fix a system of local quantum constraints $\Theta \rightarrow(\mathcal{F}(\Theta), \mathcal{U}(\Theta))$, then we say that it satisfies:

(7) weak covariance if there is an action $\alpha: \mathcal{P}_{+}^{\uparrow} \rightarrow$ Aut $\mathcal{F}_{0}$ such that $\alpha_{g}(\mathcal{O}(\Theta))=\mathcal{O}(g \Theta)$ and $\alpha_{g}(\mathcal{U}(\Theta)) \sim \mathcal{U}(g \Theta)$, for all $g \in \mathcal{P}_{+}^{\uparrow}, \Theta \in \Gamma$ (cf. Definition 3.15).

3.23 Remark (i) For the weak covariance condition, we do not need to state in which algebra the equivalence of constraints holds, since this only depends on the unital $\mathrm{C}^{*}$-algebra generated by the two constraint sets involved (cf. Remark 3.16 (ii)). Note that if the net $\Theta \rightarrow \mathcal{F}(\Theta)$ is already covariant, then weak covariance follows from the covariance condition $\alpha_{g}(\mathcal{F}(\Theta))=\mathcal{F}(g \Theta)$ and $\alpha_{g}(\mathcal{U}(\Theta)) \sim \mathcal{U}(g \Theta)$, for all $g \in \mathcal{P}_{+}^{\uparrow}$, using the fact that equivalent constraint sets produce the same observable algebra.

(ii) It is instructive to compare the conditions in Definitions 3.12 and 3.22 with those of the Doplicher-Haag-Roberts analysis (DHR for short [4]), given that both are intended for application to gauge QFTs. First, in DHR analysis one assumes that the actions of the gauge group and the Poincaré group commute, which limits the analysis to gauge transformations of the first kind (and hence excludes quantum electromagnetism). In contrast, we assume weak covariance, hence include gauge transformations of the second kind (and also QEM). The DHR analysis also assumes field algebra covariance, which we omit. Second, the DHR analysis is done concretely in a positive energy representation, whereas we assume an abstract $\mathrm{C}^{*}$-system, hence we can avoid the usual clash between regularity and constraints, which appears as continuous spectrum problems for the constraints (cf. Subsection 4.2) and which generally leads to indefinite metric representations. At the concrete level this problem manifests itself in the inability of constructing the vector potential satisfying Maxwell's equations as a covariant or causal quantum field on a space with an invariant vacuum, cf. [17, 18, 19] and [20, Eq. 8.1.2].

(iii) In the next sections we will construct an example (Gupta-Bleuler electromagnetism) which satisfies the conditions 3.12 and 3.22 .

Now we show that the conditions in Definition 3.12 and 3.22 are sufficient to guarantee that the net of local physical observables $\Theta \rightarrow \mathcal{R}(\Theta)$ is a HK-QFT. This is a central result for this paper. 
3.24 Theorem Let $\Gamma \ni \Theta \rightarrow(\mathcal{F}(\Theta), \mathcal{U}(\Theta))$ be a system of local quantum constraints. If it satisfies weak covariance, then for each $\Theta$ we have $\widetilde{\alpha}_{g}(\mathcal{R}(\Theta))=\mathcal{R}(g \Theta), g \in \mathcal{P}_{+}^{\uparrow}$, where $\widetilde{\alpha}_{g}$ is the factoring through of $\alpha_{g}$ (cf. (7) in Definition 3.22) to the local factor algebra $\mathcal{R}(\Theta)$. If the system of constraints in addition satisfies reduction isotony, then the isomorphisms $\widetilde{\alpha}_{g}: \mathcal{R}(\Theta) \rightarrow \mathcal{R}(g \Theta)$, $\Theta \in \Gamma$, are the restrictions of an automorphism $\widetilde{\alpha}_{g} \in$ Aut $\mathcal{R}_{0}$, and moreover, $\widetilde{\alpha}: \mathcal{P}_{+}^{\uparrow} \rightarrow$ Aut $\mathcal{R}_{0}$ is an action, i.e. the net $\Theta \rightarrow \mathcal{R}(\Theta)$ satisfies covariance.

Proof: Let $\alpha: \mathcal{P}_{+}^{\uparrow} \rightarrow$ Aut $\mathcal{F}_{0}$ be the action introduced by the weak covariance assumption in Definition $3.22(7)$. Now $\alpha_{g}(\mathcal{O}(\Theta))$ are the observables of the constraint system $\left(\alpha_{g}(\mathcal{F}(\Theta)), \alpha_{g}(\mathcal{U}(\Theta))\right)$ with maximal constraint algebra $\alpha_{g}(\mathcal{D}(\Theta)) \subset \alpha_{g}(\mathcal{O}(\Theta))=\mathcal{O}(g \Theta) \supset \mathcal{D}(g \Theta)$. Since $\alpha_{g}(\mathcal{U}(\Theta)) \sim$ $\mathcal{U}(g \Theta)$, they have the same Dirac states and so on $\mathcal{O}(g \Theta)$ the same maximal $\mathrm{C}^{*}$-algebra contained in the kernels of all Dirac states. Thus $\alpha_{g}(\mathcal{D}(\Theta))=\mathcal{D}(g \Theta)$. Denote the factoring map $\xi_{\Theta}: \mathcal{O}(\Theta) \rightarrow \mathcal{R}(\Theta)$, i.e. $\xi_{\Theta}(A)=A+\mathcal{D}(\Theta)$ for all $A \in \mathcal{O}(\Theta)$. Then we factor through $\alpha_{g}: \mathcal{O}(\Theta) \rightarrow \mathcal{O}(g \Theta)$ to a map $\widetilde{\alpha}_{g}: \mathcal{R}(\Theta) \rightarrow \mathcal{R}(g \Theta)$ by

$$
\widetilde{\alpha}_{g}\left(\xi_{\Theta}(A)\right):=\alpha_{g}(A)+\alpha_{g}(\mathcal{D}(\Theta))=\alpha_{g}(A)+\mathcal{D}(g \Theta)=\xi_{g \Theta}\left(\alpha_{g}(A)\right),
$$

and this is obviously an isomorphism.

Next assume in addition reduction isotony, then we show that the isomorphisms $\widetilde{\alpha}_{g}$ defined on the net $\Theta \rightarrow \mathcal{R}(\Theta)$ are the restrictions of an automorphism $\widetilde{\alpha}_{g} \in$ Aut $\mathcal{R}_{0}$. Indeed, for $\Theta_{1} \subset \Theta_{2}$ and any $A \in \mathcal{O}\left(\Theta_{1}\right)$ we have using equation $\mathcal{D}\left(g \Theta_{1}\right)=\mathcal{D}\left(g \Theta_{2}\right) \cap \mathcal{O}\left(g \Theta_{1}\right)$ and the monomorphisms $\iota_{12}: \mathcal{R}\left(\Theta_{1}\right) \rightarrow \mathcal{R}\left(\Theta_{2}\right), \iota_{12}^{g}: \mathcal{R}\left(g \Theta_{1}\right) \rightarrow \mathcal{R}\left(g \Theta_{2}\right)$ that

$$
\begin{aligned}
\iota_{12}^{g}\left(\widetilde{\alpha}_{g}\left(\xi_{\Theta_{1}}(A)\right)\right) & =\iota_{12}^{g}\left(\alpha_{g}(A)+\mathcal{D}\left(g \Theta_{1}\right)\right)=\alpha_{g}(A)+\mathcal{D}\left(g \Theta_{2}\right) \\
& =\widetilde{\alpha}_{g}\left(\xi_{\Theta_{2}}(A)\right)=\widetilde{\alpha}_{g}\left(\iota_{12}\left(\xi_{\Theta_{1}}(A)\right)\right) .
\end{aligned}
$$

This shows that the diagram

$$
\begin{array}{ccc}
\mathcal{R}\left(\Theta_{1}\right) & \stackrel{\iota_{12}}{\longrightarrow} & \mathcal{R}\left(\Theta_{2}\right) \\
\downarrow \widetilde{\alpha}_{g} & & \downarrow \widetilde{\alpha}_{g} \\
\mathcal{R}\left(g \Theta_{1}\right) & \stackrel{\iota_{12}^{g}}{\longrightarrow} & \mathcal{R}\left(g \Theta_{2}\right)
\end{array}
$$

commutes. Therefore by the uniqueness property of the inductive limit [21, Section 11.4] the isomorphisms $\widetilde{\alpha}_{g}$ of the local observable algebras characterize an automorphism of $\mathcal{R}_{0}$ which we also denote by $\widetilde{\alpha}_{g}$. Since $\alpha: \mathcal{P}_{+}^{\uparrow} \rightarrow$ Aut $\mathcal{F}$ is a group homomorphism, we see for the local isomorphisms that the composition of $\widetilde{\alpha}_{g}: \mathcal{R}(\Theta) \rightarrow \mathcal{R}(g \Theta)$ with $\widetilde{\alpha}_{h}: \mathcal{R}(g \Theta) \rightarrow \mathcal{R}(h g \Theta)$ is $\widetilde{\alpha}_{h} \circ \widetilde{\alpha}_{g}=\widetilde{\alpha}_{h g}: \mathcal{R}(\Theta) \rightarrow \mathcal{R}(h g \Theta)$. From this it follows that $\widetilde{\alpha}: \mathcal{P}_{+}^{\uparrow} \rightarrow$ Aut $\mathcal{R}_{0}$ is a group homomorphism.

So combining Theorems 3.14 and 3.24 we obtain our main claim:

3.25 Theorem If the system of local constraints satisfies all three conditions in Definitions 3.12 and 3.22, then $\Theta \rightarrow \mathcal{R}(\Theta)$ is a $H K-Q F T$.

In the following sections we will construct field theory examples of local systems of quantum constraints which satisfy the weak conditions of Definition 3.12 and 3.22, hence define HK-QFTs for their net of physical algebras. 
3.26 Proposition Given a system of local quantum constraints, $\Theta \rightarrow(\mathcal{F}(\Theta), \mathcal{U}(\Theta))$, which satisfies reduction isotony and weak covariance, then the net $\Theta \rightarrow\left(\mathcal{F}(\Theta), \mathcal{U}_{m}(\Theta)\right)\left(\right.$ where $\mathcal{U}_{m}(\Theta)$ is the maximal constraint group of $\mathcal{U}(\Theta)$ in $\mathcal{F}(\Theta)$, cf. Theorem $3.17($ ii $)$ ) is a system of local quantum constraints satisfying reduction isotony and covariance, i.e.

$$
\alpha_{g}(\mathcal{O}(\Theta))=\mathcal{O}(g \Theta) \quad \text { and } \quad \alpha_{g}\left(\mathcal{U}_{m}(\Theta)\right)=\mathcal{U}_{m}(g \Theta), \quad g \in \mathcal{P}_{+}^{\uparrow}, \Theta \in \Gamma .
$$

The system $\Theta \rightarrow\left(\mathcal{F}(\Theta), \mathcal{U}_{m}(\Theta)\right)$ is clearly locally equivalent to $\Theta \rightarrow(\mathcal{F}(\Theta), \mathcal{U}(\Theta))$, in the sense that $\mathcal{U}_{m}(\Theta) \sim \mathcal{U}(\Theta)$ for all $\Theta \in \Gamma$, from which it follows that if one of these two systems has weak causality, so has the other one.

Proof: Let $\Theta_{1} \subseteq \Theta_{2}$, then by $\left(\mathcal{F}\left(\Theta_{1}\right), \mathcal{U}\left(\Theta_{1}\right)\right) \subseteq\left(\mathcal{F}\left(\Theta_{2}\right), \mathcal{U}\left(\Theta_{2}\right)\right)$ and reduction isotony, we conclude from Theorem 3.10 that all Dirac states on $\mathcal{O}\left(\Theta_{1}\right) \subset \mathcal{O}\left(\Theta_{2}\right)$ extend to Dirac states on $\mathcal{O}\left(\Theta_{2}\right)$. Thus by Remark 3.18,

$$
\begin{aligned}
\mathcal{U}_{m}\left(\Theta_{2}\right) \cap \mathcal{F}\left(\Theta_{1}\right) & =\left\{U \in \mathcal{O}_{u}\left(\Theta_{2}\right) \mid \omega(U)=1 \quad \forall \omega \in \mathfrak{S}_{D}^{\Theta_{2}}\right\} \cap \mathcal{F}\left(\Theta_{1}\right) \\
& =\left\{U \in \mathcal{F}_{u}\left(\Theta_{1}\right) \cap \mathcal{O}\left(\Theta_{2}\right) \mid \omega(U)=1 \quad \forall \omega \in \mathfrak{S}_{D}^{\Theta_{1}}\right\} \\
& =\left\{U \in \mathcal{O}_{u}\left(\Theta_{1}\right) \mid \omega(U)=1 \quad \forall \omega \in \mathfrak{S}_{D}^{\Theta_{1}}\right\} \\
& =\mathcal{U}_{m}\left(\Theta_{1}\right)
\end{aligned}
$$

where we used the fact that if for a unitary $U$ we have $\omega(U)=1$ for all Dirac states $\omega$, then $U \in \mathcal{O}$. Thus $\left(\mathcal{F}\left(\Theta_{1}\right), \mathcal{U}_{m}\left(\Theta_{1}\right)\right) \subseteq\left(\mathcal{F}\left(\Theta_{2}\right), \mathcal{U}_{m}\left(\Theta_{2}\right)\right)$ and so the system $\Theta \rightarrow\left(\mathcal{F}(\Theta), \mathcal{U}_{m}(\Theta)\right)$ is a system of local quantum constraints. Reduction isotony follows from that of the original system and the equivalences $\mathcal{U}_{m}(\Theta) \sim \mathcal{U}(\Theta)$ for all $\Theta \in \Gamma$.

To prove the covariance property of $\mathcal{U}_{m}(\Theta)$ recall that from weak covariance we have $\alpha_{g}(\mathcal{U}(\Theta)) \sim \mathcal{U}(g \Theta)$ for all $\Theta$. We show first that if $\mathcal{U}_{1}(\Theta) \sim \mathcal{U}(\Theta)$, then $\alpha_{g}\left(\mathcal{U}_{1}(\Theta)\right) \subset \mathcal{U}_{m}(g \Theta)$. We have

$$
\begin{aligned}
\alpha_{g}\left(\mathcal{U}_{1}(\Theta)\right)-\mathcal{U}(g \Theta) & =\alpha_{g}\left(\mathcal{U}_{1}(\Theta)-\mathbb{1}\right)+\mathbb{1}-\mathcal{U}(g \Theta) \\
& \subset \alpha_{g}(\mathcal{D}(\Theta))+\mathcal{D}(g \Theta) \\
& =\mathcal{D}(g \Theta)=\alpha_{g}(\mathcal{D}(\Theta))
\end{aligned}
$$

where the last equality follows from the proof of the previous theorem, and we used also that $\mathcal{D}_{1}(\Theta)=\mathcal{D}(\Theta)$. Since $\alpha_{g}(\mathcal{D}(\Theta))$ is the $\mathcal{D}$-algebra of $\alpha_{g}\left(\mathcal{U}_{1}(\Theta)\right)$ in $\alpha_{g}(\mathcal{O}(\Theta))=\mathcal{O}(g \Theta)$, this implies by Theorem 3.17 (i) that $\alpha_{g}\left(\mathcal{U}_{1}(\Theta)\right) \sim \mathcal{U}(g \Theta)$ and therefore it must be contained in $\mathcal{U}_{m}(g \Theta)$. Thus $\alpha_{g}\left(\mathcal{U}_{m}(\Theta)\right) \subset \mathcal{U}_{m}(g \Theta), g \in \mathcal{P}$, and finally the inclusion $\alpha_{g^{-1}}\left(\mathcal{U}_{m}(g \Theta)\right) \subset \mathcal{U}_{m}(\Theta)$ proves covariance for $\Theta \rightarrow \mathcal{U}_{m}(\Theta)$.

\section{Preliminaries for the Example.}

In this section we collect the relevant material we need to develop our Gupta-Bleuler example in the next section.

\subsection{Outer constraints.}

We will need the following variant where the constraints are defined through a group action which is not necessarily inner. One assumes, following [8] that: 
- There is a distinguished group action $\beta: \mathcal{G} \rightarrow$ Aut $\mathcal{F}$ on the field algebra $\mathcal{F}$, and all physical information is contained in $\mathcal{F}$ and its set of invariant states:

$$
\mathfrak{S}^{\mathcal{G}}(\mathcal{F}):=\left\{\omega \in \mathfrak{S}(\mathcal{F}) \mid \omega\left(\beta_{g}(A)\right)=\omega(A) \quad \forall g \in \mathcal{G}, A \in \mathcal{F}\right\} .
$$

If $\mathcal{G}$ is locally compact, we can construct the (abstract) multiplier algebra of the crossed product $\mathcal{F}_{e}=M(\mathcal{G} \underset{\beta}{\times} \mathcal{F})$ and otherwise we will just take the discrete crossed product. In either case we obtain a $\mathrm{C}^{*}$-algebra $\mathcal{F}_{e} \supset \mathcal{F}$ which contains unitaries $U_{g}$ for all $g \in \mathcal{G}$ that implement $\beta: \mathcal{G} \rightarrow$ Aut $\mathcal{F}$. Then this situation is reduced to the previous one by the following theorem 8 , Section 3]:

4.1 Theorem $\mathfrak{S}^{\mathcal{G}}(\mathcal{F})$ is precisely the restriction to $\mathcal{F}$ of the Dirac states on $\mathcal{F}_{\text {e }}$ with respect to $\mathcal{C}=U_{\mathcal{G}}-1$, i.e. $\mathfrak{S}^{\mathcal{G}}(\mathcal{F})=\mathfrak{S}_{D}\left(\mathcal{F}_{e}\right) \mid \mathcal{F}$ where

$$
\mathfrak{S}_{D}\left(\mathcal{F}_{e}\right):=\left\{\omega \in \mathfrak{S}\left(\mathcal{F}_{e}\right) \mid \omega\left(U_{g}\right)=1 \quad \forall g \in \mathcal{G}\right\} .
$$

Hence we can apply the $\mathrm{T}$-procedure to $U_{\mathcal{G}}-\mathbb{1}$ in $\mathcal{F}_{e}$, and intersect the resulting algebraic structures with $\mathcal{F}$. This is called the outer constraint situation.

\subsection{Bosonic constraints.}

For free bosons, one takes for $\mathcal{F}$ the $\mathrm{C}^{*}$-algebra of the CCRs, which we now define following Manuceau [22, 23]. Let $\mathfrak{X}$ be a linear space and $B$ a (possibly degenerate) symplectic form on it. Denote by $\Delta(\mathfrak{X}, B)$ the linear space of complex-valued functions on $\mathfrak{X}$ with finite support. It has as linear basis the set $\left\{\delta_{f} \mid f \in \mathfrak{X}\right\}$, where

$$
\delta_{f}(h):=\left\{\begin{array}{lll}
1 & \text { if } \quad f=h \\
0 & \text { if } \quad f \neq h
\end{array}\right.
$$

Make $\Delta(\mathfrak{X}, B)$ into a *-algebra, by defining the product $\delta_{f} \cdot \delta_{h}:=e^{\frac{i}{2} B(f, h)} \delta_{f+h}$ and involution $\left(\delta_{f}\right)^{*}:=\delta_{-f}$, where $f, h \in S$ and the identity is $\delta_{0}$. Let $\Delta_{1}(\mathfrak{X}, B)$ be the closure of $\Delta(\mathfrak{X}, B)$ w.r.t. the norm $\left\|\sum_{i=1}^{m} \alpha_{i} \delta_{f_{i}}\right\|_{1}:=\sum_{i=1}^{m}\left|\alpha_{i}\right|, \alpha_{i} \in \mathbb{C}$, then the CCR-algebra $\overline{\Delta(\mathfrak{X}, B)}$ is defined as the enveloping $\mathrm{C}^{*}$-algebra of the the Banach *-algebra $\Delta_{1}(\mathfrak{X}, B)$. That is, it is the closure with respect to the enveloping $\mathrm{C}^{*}$-norm:

$$
\|A\|:=\sup _{\omega \in \mathfrak{S}\left(\Delta_{1}(\mathfrak{X}, B)\right)} \sqrt{\omega\left(A^{*} A\right)} .
$$

It is well-known (cf. [23]) that:

4.2 Theorem $\overline{\Delta(\mathfrak{X}, B)}$ is simple iff $B$ is nondegenerate.

An important state on $\overline{\Delta(\mathfrak{X}, B)}$ is the central state defined by

$$
\omega_{0}\left(\delta_{f}\right):= \begin{cases}1 & \text { if } f=0 \\ 0 & \text { otherwise }\end{cases}
$$

Using it, we make the following useful observations. The relation between the norms on $\Delta(\mathfrak{X}, B)$ is

$$
\|F\|_{2}:=\left(\sum_{i=1}^{n}\left|\lambda_{i}\right|^{2}\right)^{1 / 2}=\omega_{0}\left(F^{*} F\right)^{1 / 2} \leq\|F\| \leq\|F\|_{1} \text { for } F=\sum_{i=1}^{n} \lambda_{i} \delta_{f_{i}}
$$


and hence $\Delta_{1}(\mathfrak{X}, B) \subset \overline{\Delta(\mathfrak{X}, B)} \subset \ell^{2}(\mathfrak{X})$, so we can write an $A \in \overline{\Delta(\mathfrak{X}, B)}$ as $A=\sum_{i=1}^{\infty} \lambda_{i} \delta_{f_{i}}$ where $\left\{\lambda_{i}\right\} \in \ell^{2}$ and $f_{i}=f_{j}$ iff $i=j$. Let $A_{n}:=\sum_{i=1}^{M_{n}} \gamma_{i}^{(n)} \delta_{f_{i}^{(n)}} \subset \Delta(\mathfrak{X}, B)$ converge to $A \in \overline{\Delta(\mathfrak{X}, B)}$ in $\mathrm{C}^{*}$-norm then since the family of $f_{i}^{(n)}$ is denumerable we can arrange it into a single sequence $f_{i}$ and thus write $A_{n}:=\sum_{i=1}^{N_{n}} \lambda_{i}^{(n)} \delta_{f_{i}}$. We shall frequently use this way of writing a Cauchy sequence in $\Delta(\mathfrak{X}, B)$.

Now to define a constrained system corresponding to linear selfadjoint constraints in $\mathcal{F}=$ $\overline{\Delta(\mathfrak{X}, B)}$, we choose a set $\mathcal{C}=\mathcal{U}-\mathbb{1}$ where $\mathcal{U}=\left\{\delta_{f} \mid f \in \mathfrak{s}\right\}$ and $\mathfrak{s} \subset \mathfrak{X}$ is a subspace corresponding to the "test functions" of the heuristic constraints.

4.3 Theorem Define the symplectic commutant $\mathfrak{s}^{\prime}:=\{f \in \mathfrak{X} \mid B(f, \mathfrak{s})=0\}$, then $\mathcal{C}=\mathcal{U}-\mathbb{1}$ is first class iff $\mathfrak{s} \subseteq \mathfrak{s}^{\prime}$.

For the proof, see Lemma 6.1 in [10].

We saw after Theorem 2.3 that for the observable algebra we sometimes need to choose a smaller algebra $\mathcal{O}_{c} \subset \mathcal{O}$ in order to ensure that the physical algebra $\mathcal{R}_{c}$ is simple. For bosonic constraints with nondegenerate $B$, such an algebra is $\mathcal{O}_{c}=\mathrm{C}^{*}\left(\delta_{\mathfrak{s}^{\prime}}\right)=\mathcal{C}^{\prime}$ (in which case $\mathcal{D} \cap \mathcal{O}_{c}=$ $\left.\mathrm{C}^{*}\left(\delta_{\mathfrak{s}^{\prime}}\right) \cdot \mathrm{C}^{*}\left(\delta_{\mathfrak{s}}-\mathbb{1}\right)\right)$, which is what was chosen in [3, 10]. However, we now show that with this choice we have in fact $\mathcal{R}_{c}=\mathcal{R}$, i.e. we obtain the same physical algebra than with the full $\mathrm{T}$-procedure, so nothing was lost by this choice of $\mathcal{O}_{c}$.

4.4 Theorem Given nondegenerate $(\mathfrak{X}, B)$ and $\mathfrak{s} \subset \mathfrak{X}$ as above, where $\mathfrak{s} \subset \mathfrak{s}^{\prime}$ and $\mathfrak{s}=\mathfrak{s}^{\prime \prime}$, then

$$
\mathcal{O}=\mathrm{C}^{*}\left(\delta_{\mathfrak{s}^{\prime}} \cup \mathcal{D}\right)=\left[\mathrm{C}^{*}\left(\delta_{\mathfrak{s}^{\prime}}\right) \cup \mathcal{D}\right]
$$

Proof: The proof of this is new but long, so we put it in Appendix 2.

4.5 Theorem Consider a nondegenerate symplectic space $(\mathfrak{X}, B)$ and a first class set $\mathfrak{s} \subset \mathfrak{X}$. Denote by $\widetilde{B}$ the factoring through of $B$ to the factor space $\mathfrak{s}^{\prime} / \mathfrak{s}$. Then we have the following isomorphism:

$$
\mathrm{C}^{*}\left(\delta_{\mathfrak{s}^{\prime}}\right) / \mathrm{C}^{*}\left(\delta_{\mathfrak{s}}-\mathbb{1}\right) \mathrm{C}^{*}\left(\delta_{\mathfrak{s}^{\prime}}\right) \cong \overline{\Delta\left(\mathfrak{s}^{\prime} / \mathfrak{s}, \widetilde{B}\right)} .
$$

In particular, if $\mathfrak{s}=\mathfrak{s}^{\prime \prime}$, then $\left(\mathfrak{s}^{\prime} / \mathfrak{s}, \widetilde{B}\right)$ is nondegenerate, so the above CCR-algebra is simple, and using Theorem 4.4 we have

$$
\mathcal{R} \cong \overline{\Delta\left(\mathfrak{s}^{\prime} / \mathfrak{s}, \widetilde{B}\right)}
$$

For proofs and further details see $\mathbb{8}$, Theorem 5.2 and 5.3, as well as Corollary 5.4 and 5.5]. The surprise is that for linear bosonic constrained systems, the choice of traditional observables $\mathcal{O}_{c}=\mathcal{C}^{\prime}$ produces the same physical algebra $\mathcal{R}$ than the $\mathrm{T}$-procedure, which is not true in general.

A typical pathology which occurs for bosonic constraints, is that the Dirac states are not regular, i.e. the one parameter groups $\mathbb{R} \ni t \mapsto \pi_{\omega}\left(\delta_{t f}\right)$ for $\omega \in \mathfrak{S}_{D}$ will not be strong operator continuous for all $f \in \mathfrak{X}$, and so the corresponding generators (which are the smeared quantum fields in many models of bosonic fields), will not exist for some $f \in \mathfrak{X}$, cf. [24]. The resolution of this, is that the pathology only occurs on nonphysical elements, i.e. on $\delta_{f} \notin \mathcal{O}$, with the result that a Dirac state when restricted to $\mathcal{O}$ and factored to $\mathcal{R}$ (i.e. taken through the bijection in Theorem 2.4) can be regular again on the physical algebra $\mathcal{R}$. This is also obvious from Theorem 4.5. since a nontrivial $\mathcal{R}$ clearly has regular states. Thus for the physical algebra, quantum fields can exist. 


\section{Example: Gupta-Bleuler electromagnetism}

Quantum electromagnetism, in the heuristic Gupta-Bleuler formulation, has a number of special features, cf. 25, 26]. First, it is represented on an indefinite inner product space, second, gauge invariance is imposed by the noncausal constraint

$$
\chi(x):=\left(\partial^{\mu} A_{\mu}\right)^{(+)}(x),
$$

and third, Maxwell's equations (in terms of the vector potential) are imposed as state conditions instead of as operator identities. This is necessary, because from the work of Strocchi (e.g. [27, 7]), we know that Maxwell's equations are incompatible with the Lorentz covariance of the vector potential. Gupta-Bleuler electromagnetism has been rigorously reconstructed in a $\mathrm{C}^{*}$-algebra context [8], in a way which allows one to avoid indefinite inner product representations (using instead representations which are nonregular on nonphysical objects). Here we will refine that approach in order to include the local constraint structure and to make contact with Haag-Kastler QFT. Our aim is to define Gupta-Bleuler electromagnetism as a local system of constraints as in Definition 3.3 and subsequently to show that it has reduction isotony, weak causality and covariance. Our starting point for defining this system, is [8, Sections 4 and 5] where motivation and further results can be found.

\subsection{Gupta-Bleuler electromagnetism - the heuristic theory.}

Heuristically the field is

$$
F_{\mu \nu}(x):=A_{\nu, \mu}(x)-A_{\mu, \nu}(x)
$$

where the vector potential, constructed on a Fock-Krein space $\mathcal{H}$ is:

$$
A_{\mu}(x)=\left(2(2 \pi)^{3}\right)^{-\frac{1}{2}} \int_{C_{+}}\left(a_{\mu}(\mathbf{p}) e^{-i p \cdot x}+a_{\mu}^{\dagger}(\mathbf{p}) e^{i p \cdot x}\right) \frac{d^{3} p}{p_{0}}
$$

where $C_{+}:=\left\{p \in \mathbb{R}^{4} \mid p_{\mu} p^{\mu}=0, p_{0} \geq 0\right\}$ is the mantle of the positive light cone: $V_{+}:=\{p \in$ $\left.\mathbb{R}^{4} \mid p_{\mu} p^{\mu} \geq 0, p_{0} \geq 0\right\}$. Note that the adjoints $a^{\dagger}$ are w.r.t. the indefinite inner product, and that the latter comes from the indefinite inner product on the one particle space:

$$
K(f, h):=-2 \pi \int_{C_{+}} \frac{d^{3} p}{p_{0}} \bar{f}_{\mu}(p) h^{\mu}(p) .
$$

Then the CCR's are

$$
\left[A_{\mu}(x), A_{\nu}\left(x^{\prime}\right)\right]=-i \eta_{\mu \nu} D\left(x-x^{\prime}\right), \quad D(x):=-(2 \pi)^{-3} \int_{C_{+}} e^{i \mathbf{p} \cdot \mathbf{x}} \sin \left(p_{0} x_{0}\right) \frac{d^{3} p}{p_{0}}
$$

using $\left[a_{\mu}(\mathbf{p}), a_{\nu}^{\dagger}\left(\mathbf{p}^{\prime}\right)\right]=-\eta_{\mu \nu}\|\mathbf{p}\| \delta^{3}\left(\mathbf{p}-\mathbf{p}^{\prime}\right)$ and the other commutators involving $a$ are zero. At this point $A_{\mu}(x)$ does not yet satisfy the field equations $F_{\mu \nu}, \nu(x)=0$. On smearing we obtain:

$$
\begin{aligned}
A(\hat{f}) & :=\int d^{4} x A_{\mu}(x) f^{\mu}(x) \\
& =\sqrt{\pi} \int_{C_{+}}\left(a_{\mu}(\mathbf{p}) \hat{f}^{\mu}(p)+a_{\mu}^{\dagger}(\mathbf{p}) \overline{\hat{f}^{\mu}(p)}\right) \frac{d^{3} p}{p_{0}} \\
& =\left(a(f)+a(f)^{\dagger}\right) / \sqrt{2} \\
\text { with } \quad a(f) & :=\sqrt{2 \pi} \int_{C_{+}} a_{\mu}(\mathbf{p}) \hat{f}^{\mu}(p) \frac{d^{3} p}{p_{0}}
\end{aligned}
$$


where $f \in \mathcal{S}\left(\mathbb{R}^{4}, \mathbb{R}^{4}\right)$ and $\hat{f}_{\mu}(p):=(2 \pi)^{-2} \int d^{4} x e^{-i p \cdot x} f_{\mu}(x) \in \widehat{\mathcal{S}}$ and the latter means

$$
\widehat{\mathcal{S}}:=\left\{\widehat{f} \mid f \in \mathcal{S}\left(\mathbb{R}^{4}, \mathbb{R}^{4}\right)\right\}=\left\{f \in \mathcal{S}\left(\mathbb{R}^{4}, \mathbb{C}^{4}\right) \mid \overline{f(p)}=f(-p)\right\}
$$

and as usual $\mathcal{S}$ denotes Schwartz functions. The operators $A(\hat{f})$ are Krein symmetric, but not selfadjoint. Then the smeared CCRs are

$$
[A(f), A(h)]=i D(f, h):=-\pi \int_{C_{+}}\left(f_{\mu}(p) \overline{h^{\mu}}(p)-\overline{f_{\mu}}(p) h^{\mu}(p)\right) \frac{d^{3} p}{p_{0}} .
$$

Note that the distribution $D$ is actually the Fourier transform of the usual Pauli-Jordan distribution, i.e.

$$
\widehat{D}(f, h):=D(\widehat{f}, \widehat{h})=\iint f_{\mu}(x) h^{\mu}(y) D(x-y) d^{4} x d^{4} y
$$

in heuristic form. The supplementary condition

$$
\chi(x):=\partial^{\mu} A_{\mu}^{(+)}(x)=-i\left(2(2 \pi)^{3}\right)^{-\frac{1}{2}} \int_{C_{+}} p^{\mu} a_{\mu}(\mathbf{p}) e^{-i p \cdot x} \frac{d^{3} p}{p_{0}}
$$

selects the physical subspace $\mathcal{H}^{\prime}:=\left\{\psi \in \mathcal{H} \mid \chi(h) \psi=0, h \in \mathcal{S}\left(\mathbb{R}^{4}, \mathbb{R}\right)\right\}$ (to make this welldefined, we need to specify the domain of $\chi(h)$,- this will be done in Subsection 5.6). The Poincaré transformations are defined in the natural way: $(\Lambda, a) f(p)=e^{i a \cdot p} \Lambda f\left(\Lambda^{-1} p\right)$, and the given Krein inner product on $\mathcal{H}$ is invariant w.r.t. the Poincaré transformations, but not the Hilbert inner product. Moreover $\mathcal{H}^{\prime}$ is positive semidefinite w.r.t. the Krein inner product $\langle\cdot, \cdot\rangle$, so the heuristic theory constructs the physical Hilbert space $\mathcal{H}_{\text {phys }}$ as the closure of $\mathcal{H}^{\prime} / \mathcal{H}^{\prime \prime}$ equipped with inner product $\langle\cdot, \cdot\rangle$ where $\mathcal{H}^{\prime \prime}$ is the zero norm part of it. At the one particle level, $\mathcal{H}^{\prime}$ consists of functions satisfying $p_{\mu} f^{\mu}(p)=0$, and $\mathcal{H}^{\prime \prime}$ consists of gradients $f_{\mu}(p)=i p_{\mu} h(p)$. The physical observables consist of operators which can factor to $\mathcal{H}_{\text {phys }}$, and in particular contains the field operators $F_{\mu \nu}$. These satisfy the Maxwell equations on $\mathcal{H}_{\text {phys }}$, because $F_{\mu \nu}{ }^{, \mu}$ maps $\mathcal{H}^{\prime}$ to $\mathcal{H}^{\prime \prime}$.

Note that since the Krein inner product becomes the Hilbert inner product on $\mathcal{H}_{\text {phys }}$, the Krein adjoint becomes the Hilbert space adjoint for physical observables. With this in mind, we will below do a reconstruction in $\mathrm{C}^{*}$-algebraic terms where the $\mathrm{C}^{*}$-involution corresponds to the Krein involution.

\subsection{Gupta-Bleuler electromagnetism as a local constraint system.}

To model this in rigorous field theory, we start with the CCR algebra $\mathcal{A}:=\overline{\Delta(\mathfrak{X}, B)}$ where the symplectic space $(\mathfrak{X}, B)$ is constructed as follows. Consider the real linear space $\widehat{\mathcal{S}}$ from above, and equip it with the presymplectic form $D$ obtained from the CCRs before. Now define $\mathfrak{X}:=\widehat{\mathcal{S}} / \operatorname{Ker}(D)$ which is a symplectic space with symplectic form $B$ defined as the factoring of $D$ to the factor space $\mathfrak{X}$.

Now since the constraints $\chi(x)$ are not (Krein) selfadjoint, there is no space of test functions in $\mathfrak{X}$ which represent them, so we want to define them as outer constraints through the gauge transformations which they generate. A heuristic calculation (cf. [8]) produces:

$$
\operatorname{Ad}\left(\exp \left(-i t \chi(h)^{\dagger} \chi(h)\right)\right) \exp (i A(f))=\exp \left(i A\left(T_{h}^{t} f\right)\right)
$$

where formally $\chi(h):=\int \chi(x) h(x) d^{4} x, h \in \mathcal{S}\left(\mathbb{R}^{4}, \mathbb{R}\right)$ and

$$
\left(T_{h}^{t} f\right)_{\mu}(p)=f_{\mu}(p)-i t \pi p_{\mu} \widehat{h}(p) \int_{C_{+}} f^{\nu}\left(p^{\prime}\right) p_{\nu}^{\prime} \overline{\widehat{h}\left(p^{\prime}\right)} \frac{d^{3} p^{\prime}}{p_{0}^{\prime}}
$$


and we used the smearing formula Eqn (6). (Note that since the operators $A(f)$ are not selfadjoint, the operators $\exp (i A(f))$ can be unbounded). At this point a problem occurs (pointed out to us by Prof. D. Buchholz) 円. Whilst the function $i p_{\mu} \widehat{h}(p)$ is the Fourier transformation of the gradient of $h$, hence in the allowed class of functions, the coefficient $c(f, h):=\int_{C_{+}} f^{\nu}\left(p^{\prime}\right) p_{\nu}^{\prime} \overline{\widehat{h}\left(p^{\prime}\right)} \frac{d^{3} p^{\prime}}{p_{0}^{\prime}}$ need not be real, so $T_{h}^{t}$ will not preserve $\mathfrak{X}$. The reason for this difficulty, is because $\chi(h)^{\dagger} \chi(h)$ is a product of noncausal operator-valued distributions, and so its commutator with the causal $A(f)$ is unlikely to be causal. So, since the gauge transformations can take an $f \in \mathfrak{X}$ out of $\mathfrak{X}$, we enlarge the space $\mathfrak{X}$ by including complex valued Schwartz functions, i.e. we set

$$
\mathfrak{Y}:=\mathcal{S}\left(\mathbb{R}^{4}, \mathbb{C}^{4}\right) \widehat{\operatorname{Ker}}(D)=\mathcal{S}\left(\mathbb{R}^{4}, \mathbb{C}^{4}\right) / \operatorname{Ker}(D)
$$

where $D$ is given by the same formula $(\mathbb{7})$ than before;- it is still real on $\mathfrak{Y}$ because it is the imaginary part of $K$. We will discuss in Remark 5.4(ii) below what this enlargement of symplectic space corresponds to in terms of the original heuristic smearing formulii. (Note however, that the symplectic form $D$ given by Eqn (7) for arbitrary complex Schwartz functions, does not satisfy causality.) Since $\operatorname{Ker}(D \mid \widehat{\mathcal{S}})=\widehat{\mathcal{S}} \cap \operatorname{Ker}\left(D \mid \mathcal{S}\left(\mathbb{R}^{4}, \mathbb{C}^{4}\right)\right)$, we have that $\mathfrak{X} \subset \mathfrak{Y}$. Thus $\overline{\Delta(\mathfrak{X}, B)} \subset$ $\overline{\Delta(\mathfrak{Y}, B)}$, and moreover the gauge transformations $T_{h}^{t}$ are well defined on $\mathfrak{Y}$. The transformations $T_{h}^{t}$ are symplectic, in fact, if we define $G_{h}(f):=T_{h}^{1}(f)-f$, then

(i) $B\left(G_{h}(f), k\right)=-B\left(f, G_{h}(k)\right)$,

(ii) $G_{g} \circ G_{h}=0$,

(iii) $T_{h}^{t}\left(T_{k}^{s}(f)\right)=f+t G_{h}(f)+s G_{k}(f)$.

For each $h \in \mathcal{S}\left(\mathbb{R}^{4}, \mathbb{R}\right)$ we have a one-parameter group of gauge transformations $T_{h}^{t}: \mathcal{S}\left(\mathbb{R}^{4}, \mathbb{C}^{4}\right) \rightarrow$ $\mathcal{S}\left(\mathbb{R}^{4}, \mathbb{C}^{4}\right)$ (cf. $\| 8$ ) and $\left\{T_{h}^{t} \mid t \in \mathbb{R}, h \in \mathcal{S}\left(\mathbb{R}^{4}, \mathbb{R}\right)\right\}$ is a commutative set of symplectic transformations, hence preserve $\operatorname{Ker}(D)$ and so factor to the space $\mathfrak{Y}$. Each $T_{h}^{t}$ is a one-parameter group in $t$, but due to the nonlinearity in $h$, the map $h \rightarrow T_{h}^{1}=: T_{h}$ is not a group homomorphism of $\mathcal{S}\left(\mathbb{R}^{4}, \mathbb{R}\right)$.

We let our group of gauge transformations $\mathcal{G}$, be the discrete group generated in $\operatorname{Sp}(\mathfrak{Y}, B)$ by all $T_{h}^{t}$, and define as usual the action $\beta: \mathcal{G} \rightarrow \operatorname{Aut}(\overline{\Delta(\mathfrak{Y}, B)})$ by $\beta_{\gamma}\left(\delta_{f}\right)=\delta_{\gamma(f)}, \gamma \in \mathcal{G}, f \in \mathcal{Y}$. Our field algebra will be the discrete crossed product $\mathcal{F}_{e}:=\mathcal{G} \underset{\beta}{\times} \overline{\Delta(\mathfrak{Y}, B)}$. As a $\mathrm{C}^{*}$-algebra $\mathcal{F}_{e}$ is generated by $\overline{\Delta(\mathfrak{Y}, B)}$ and a set of commuting unitaries $U_{\mathcal{G}}:=\left\{U_{\gamma} \mid \gamma \in \mathcal{G}\right\}$ such that $\gamma(F)=U_{\gamma} F U_{\gamma}^{*}, F \in \mathcal{A}, U_{\gamma^{-1}}=U_{\gamma}^{*}$ and $U_{\gamma \gamma^{\prime}}=U_{\gamma} U_{\gamma^{\prime}}, \gamma, \gamma^{\prime} \in \mathcal{G}$.

5.1 Remark (i) Sometimes we need a more concrete characterization of the space $\mathfrak{X}$. Now $\mathfrak{X}=\widehat{\mathcal{S}} / \operatorname{Ker}(D)$ and $\widehat{\mathcal{S}}=\left\{f \in \mathcal{S}\left(\mathbb{R}^{4}, \mathbb{C}^{4}\right) \mid \overline{f(p)}=f(-p)\right\}=\mathcal{S}_{+}+i \mathcal{S}_{-}$where $\mathcal{S}_{ \pm}:=\left\{u \in \mathcal{S}\left(\mathbb{R}^{4}, \mathbb{R}^{4}\right) \mid u(p)= \pm u(-p)\right\}$. From Eqn. (可) we see that $\operatorname{Ker}(D)=$ $\left\{f \in \widehat{\mathcal{S}} \mid f \nmid C_{+}=0\right\}$, and hence factoring by $\operatorname{Ker}(D)$ is the same as restriction to $C_{+}$, i.e. $\mathfrak{X}=\widehat{\mathcal{S}}\left\lceil C_{+}\right.$, and since $f(p) \mid C_{+}=f(\|\mathbf{p}\|, \mathbf{p})$ we can identify these functions with a subspace of $C\left(\mathbb{R}^{3}, \mathbb{C}^{4}\right)$. Since we are restricting Schwartz functions, we note that these functions on $\mathbb{R}^{3}$ are smooth except at the origin, and Schwartz on the complement of any open neighbourhood of the origin. The conditions $u(p)= \pm u(-p)$ for $u \in \mathcal{S}_{ \pm}$involve points outside $C_{+}$, so through smoothness they will influence the behaviour of $u \uparrow C_{+}$near the origin. Specifically if $u \in \mathcal{S}_{+}$(resp. $u \in \mathcal{S}_{-}$), then on each line through the origin in $C_{+},\{t a \mid t \in \mathbb{R}\}, a \in C_{+} \backslash 0$, the function $u_{a}(t):=u(t a)$ is smooth and even (resp. odd), hence all its derivatives of odd degree must be odd (resp. even) and its derivatives of even degree must be even (resp. odd). Thus the derivatives of $u_{a}$ of odd (resp. even) degree are zero at the origin. This is

\footnotetext{
${ }^{1}$ This was also an error in $[8]$.
} 
a property which does restrict to $C_{+}$, and distinguishes between $\mathcal{S}_{+}\left\lceil C_{+}\right.$and $\mathcal{S}_{-}\left\lceil C_{+}\right.$. Note from the above discussion, that $\mathfrak{X}=\widehat{\mathcal{S}} \uparrow C_{+}$contains all smooth functions with compact support away from zero.

(ii) The space to which we will next restrict our constructions, is the real span of the orbit of $\mathfrak{X}$ under the gauge group $\mathcal{G}$, i.e. $\mathfrak{Z}:=\operatorname{Span}_{\mathbb{R}}(\mathcal{G}(\mathfrak{X}))$. Denote the real space of gradients by

$$
\mathfrak{G}:=\left\{f \in \widehat{\mathcal{S}} \mid f_{\mu}(p)=i p_{\mu} \widehat{h}(p), h \in \mathcal{S}\left(\mathbb{R}^{4}, \mathbb{R}\right)\right\}
$$

(which is not in $\operatorname{Ker}(D)$ ). Now we want to show that $\mathfrak{Z}=\mathfrak{X}+\mathbb{C} \cdot \mathfrak{G}$ where we use the same symbol for $\mathfrak{G}$ and its image in $\mathfrak{Y}$ under factoring by $\operatorname{Ker}(D)$, and $\mathbb{C} \cdot \mathfrak{G}$ is a shorthand for the complex span $\operatorname{Span}_{\mathbb{C}}(\mathfrak{G})$. Note that a general element of $\mathfrak{Z}$ is of the form

$$
\begin{aligned}
\sum_{n=1}^{N} \lambda_{n}\left(f_{\mu}^{(n)}(p)-i t_{n} \pi p_{\mu} \widehat{h}^{(n)}(p) \cdot c\left(h^{(n)}, f^{(n)}\right)\right) \\
\quad=\sum_{n=1}^{N} \lambda_{n} f_{\mu}^{(n)}(p)-i p_{\mu} \sum_{n=1}^{N} \pi t_{n} \lambda_{n} c\left(h^{(n)}, f^{(n)}\right) \cdot \widehat{h}^{(n)}(p)
\end{aligned}
$$

where $\lambda_{n}, t_{n} \in \mathbb{R}, f^{(n)} \in \mathfrak{X}, h^{(n)} \in \mathcal{S}\left(\mathbb{R}^{4}, \mathbb{R}\right)$ and $c(h, f) \in \mathbb{C}$ as in Eqn. (8). Clearly this shows that $\mathfrak{Z} \subseteq \mathfrak{X}+\mathbb{C} \cdot \mathfrak{G}$. For the reverse inclusion, we have that $\mathfrak{X}$ is in $\mathfrak{Z}$ and to see that $\mathbb{C} \cdot \mathfrak{G}$ is in $\mathfrak{Z}$, note that it contains $\pi^{-1}\left(T_{h}^{t} f-T_{h}^{t+1} f\right)(p)=i p_{\mu} \widehat{h}(p) \cdot c(f, h)$ for all $f$ and $h$. From the discussion in the previous remark, it is clear that we may choose the real and imaginary parts of $f$ and $\widehat{h}$ independently, and so $c(f, h)$ can be any complex number. Thus $\mathfrak{Z}=\mathfrak{X}+\mathbb{C} \cdot \mathfrak{G}$. From a physical point of view, one can justify the inclusion of complex smearing functions in $\mathfrak{Z}$ by the fact that the constraints $\chi(f)$ are already noncausal, and that below for the final physical theory we will eliminate these, retaining only the real valued smearing functions.

To construct the net of local field algebras $\mathcal{F}: \Gamma \rightarrow \widetilde{\Gamma}$ as in Definition 3.1, let $\Theta$ be any open set in $\mathbb{R}^{4}$ and define

$$
\begin{aligned}
\mathcal{S}(\Theta) & :=\left\{f \in \mathcal{S}\left(\mathbb{R}^{4}, \mathbb{C}^{4}\right) \mid \operatorname{supp}(f) \subset \Theta\right\} \\
\mathfrak{X}(\Theta) & :=(\widehat{\mathcal{S}(\Theta)} \cap \widehat{\mathcal{S}}) / \operatorname{Ker}(D) \\
\mathcal{Z}(\Theta) & :=(\widehat{\mathcal{S}(\Theta)} / \operatorname{Ker}(D)) \cap \mathfrak{Z} \\
\mathcal{U}(\Theta) & :=\left\{U_{T_{h}} \mid h \in \mathcal{S}\left(\mathbb{R}^{4}, \mathbb{R}\right), \operatorname{supp}(h) \subset \Theta\right\} \\
\mathcal{F}(\Theta) & :=\mathrm{C}^{*}\left(\delta_{\mathfrak{X}(\Theta)} \cup \mathcal{U}(\Theta)\right) \subset \mathcal{F}_{e} .
\end{aligned}
$$

Note that if $\Theta$ is bounded, then $\mathcal{S}(\Theta)=C_{c}^{\infty}\left(\Theta, \mathbb{C}^{4}\right)$. Moreover $T_{h} \mathfrak{Z}(\Theta) \subset \mathfrak{Z}(\Theta)$ when $\operatorname{supp}(h) \subset$ $\Theta$. Thus if we let $\mathcal{G}(\Theta)$ be the discrete group generated in $\operatorname{Sp}(\mathfrak{Y}, B)$ by $\left\{T_{h}^{t} \mid \operatorname{supp}(h) \subset \Theta, t \in\right.$ $\mathbb{R}\}$, then it preserves $\mathrm{C}^{*}\left(\delta_{\mathfrak{Z}(\Theta)}\right)$ so that it makes sense to define $\mathcal{G}(\Theta) \underset{\beta}{\times} \mathrm{C}^{*}\left(\delta_{\mathfrak{Z}(\Theta)}\right)$.

5.2 Lemma We have:

$$
\mathcal{F}(\Theta)=\mathcal{G}(\Theta) \underset{\beta}{\times} \mathrm{C}^{*}\left(\delta_{\mathfrak{Z}(\Theta)}\right)=\left[U_{\mathcal{G}(\Theta)} \delta_{\mathfrak{Z}(\Theta)}\right]=\left[\delta_{\mathfrak{Z}(\Theta)} U_{\mathcal{G}(\Theta)}\right]
$$

Proof: We start with the proof of the first equality. From $\delta_{\mathfrak{X}(\Theta)}$ and $\mathcal{U}(\Theta)$ we can produce $\delta_{\mathcal{G}(\Theta)(\mathfrak{X}(\Theta))}$ in $\mathcal{F}(\Theta)$. Let $g=T_{h_{1}}^{t_{1}} \cdots T_{h_{n}}^{t_{n}} \in \mathcal{G}(\Theta)$, then $g(f)=f+\sum_{i}^{n} t_{i} G_{h_{i}}(f) \in \mathfrak{Z}(\Theta)$ where $f \in \mathfrak{X}(\Theta), \operatorname{supp}\left(h_{i}\right) \subset \Theta$, and $G_{h}(f):=T_{h}(f)-f \in \mathbb{C} \cdot \mathfrak{G} \cap \widehat{\mathcal{S}(\Theta)}$. By varying the $h_{i}$ we can get all possible complex multiples of the gradients in $\mathfrak{G} \cap \widehat{\mathcal{S}(\Theta)}$, hence $\mathcal{G}(\Theta)(\mathfrak{X}(\Theta))=\mathfrak{Z}(\Theta)$. Thus 
$\mathcal{F}(\Theta)=\mathrm{C}^{*}\left(\delta_{\mathfrak{Z}(\Theta)} \cup \mathcal{U}(\Theta)\right)$. Now recall the fact that the crossed product $\mathcal{G}(\Theta){ }_{\beta}^{\times} \mathrm{C}^{*}\left(\delta_{\mathfrak{Z}(\Theta)}\right)$ is constructed from the twisted (by $\beta$ ) convolution algebra of functions $f: \mathcal{G}(\Theta) \rightarrow \mathrm{C}^{*}\left(\delta_{\mathfrak{Z}(\Theta)}\right)$ of finite support, and these form a subalgebra of $\mathcal{F}_{e}$. The enveloping $\mathrm{C}^{*}$-norm on this convolution algebra coincides with the $\mathrm{C}^{*}$-norm of $\mathcal{F}_{e}$, and now the equality follows from the fact that the *-algebra $\mathcal{A}(\Theta)$ generated by $\left\{\delta_{\mathfrak{Z}(\Theta)} \cup \mathcal{U}(\Theta)\right\}$ is dense in this convolution algebra. For the next two equalities note that $\mathcal{A}(\Theta)$ consists of linear combinations of products of unitaries in $\delta_{\mathfrak{Z}(\Theta)}$ and unitaries in $\mathcal{U}(\Theta)$. Each such a product of unitaries can be written as a constant times a product of the form $U_{\gamma} \cdot \delta_{f}, \gamma \in \mathcal{G}, f \in \mathfrak{Z}(\Theta)$ as well as a product of the form $\delta_{f^{\prime}} \cdot U_{\gamma^{\prime}}$, using the Weyl relation together with the implementing relation $U_{\gamma} \delta_{f}=\delta_{\gamma(f)} U_{\gamma}$ to rearrange the order of the products. Clearly now the last two relations follow from this.

By setting $\Theta=\mathbb{R}^{4}$, the global objects are included in this lemma. Also observe that whilst $U_{\mathcal{G}(\Theta)}$ is clearly an equivalent set of constraints to $\mathcal{U}(\Theta)$, in general it is strictly larger as a set. Now to define a system of local quantum constraints (cf. Def. 3.3) let $\Gamma$ be any directed set of open bounded sets of $\mathbb{R}^{4}$ which covers $\mathbb{R}^{4}$, and such that orthochronous Poincaré transformations map elements of $\Gamma$ to elements of $\Gamma$. Then the map $\mathcal{F}$ from $\Gamma$ to subalgebras of $\mathcal{F}_{e}$ by $\Theta \rightarrow \mathcal{F}(\Theta)$ satisfies isotony. The main result of this subsection is:

5.3 Theorem The map $\Gamma \ni \Theta \rightarrow(\mathcal{F}(\Theta), \mathcal{U}(\Theta))$ defines a system of local quantum constraints.

Proof: The net $\Theta \rightarrow \mathcal{F}(\Theta)$ is isotone and by construction of the cross product we also have $\mathcal{U}\left(\Theta_{1}\right)=\mathcal{U}\left(\Theta_{2}\right) \cap \mathcal{F}\left(\Theta_{1}\right)$ if $\Theta_{1} \subseteq \Theta_{2}$. It remains to show that $\mathcal{U}(\Theta)$ is first class in $\mathcal{F}(\Theta), \Theta \in \Gamma$. Consider the central state $\omega_{0}$ on $\mathrm{C}^{*}\left(\delta_{\mathfrak{Z}}\right)$ (cf. Eqn. (14)). This is $\mathcal{G}$-invariant, and its restriction to $\mathrm{C}^{*}\left(\delta_{\mathfrak{Z}(\Theta)}\right)$ is clearly $\mathcal{G}(\Theta)$-invariant. By Theorem 4.1 it extends to a nontrivial Dirac state on $\mathcal{F}(\Theta)$, hence $\mathcal{U}(\Theta)$ is first-class.

5.4 Remark (i) Observe that as $\Gamma$ is preserved by translations (cf. Definition 3.1), we can cover each compact set in $\mathbb{R}^{4}$ by a finite number of elements in $\Gamma$. Hence, since $\Gamma$ is a directed set, each compact set in $\mathbb{R}^{4}$ is contained in an element of $\Gamma$. Thus $\cup\{\mathcal{S}(\Theta) \mid \Theta \in \Gamma\}=$ $C_{c}^{\infty}\left(\mathbb{R}^{4}, \mathbb{C}^{4}\right)$ and so

$$
\mathcal{F}_{0}=\lim _{\longrightarrow} \mathcal{F}(\Theta)=\mathrm{C}^{*}\left(\delta_{\mathfrak{Z}_{(0)}} \cup \mathcal{U}_{(0)}\right) \subset \mathcal{F}_{e}
$$

where $\mathfrak{Z}_{(0)}:=\mathfrak{Z} \cap C_{c}^{\infty}\left(\mathbb{R}^{4}, \mathbb{C}^{4}\right)^{\widehat{\gamma}} / \operatorname{Ker} D$ and $\mathcal{U}_{(0)}:=\left\{U_{T_{h}} \mid h \in C_{c}^{\infty}\left(\mathbb{R}^{4}, \mathbb{R}\right)\right\}$.

(ii) Having now constructed the proposed algebraic framework for Gupta-Bleuler electromagnetism, we still need to justify the extension of our symplectic space by complex test functions. From the heuristic smearing formulii, it seems that there are two inequivalent ways of extending the smearing to complex functions, depending on whether one generalises Eqn (5) or Eqn (6). Specifically, for a complex-valued test function $f$, one has the choice of

$$
\begin{aligned}
A_{1}(\hat{f}) & :=\int d^{4} x A_{\mu}(x) f^{\mu}(x)=\left(a(f)+a(\bar{f})^{\dagger}\right) / \sqrt{2} \\
\text { or: } \quad A_{2}(\hat{f}) & :=\sqrt{\pi} \int_{C_{+}}\left(a_{\mu}(\mathbf{p}) \hat{f}^{\mu}(p)+a_{\mu}^{\dagger}(\mathbf{p}) \overline{\hat{f}^{\mu}(p)}\right) \frac{d^{3} p}{p_{0}} \\
& =\left(a(f)+a(f)^{\dagger}\right) / \sqrt{2} \\
\text { with } \quad a(f) & :=\sqrt{2 \pi} \int_{C_{+}} a_{\mu}(\mathbf{p}) \hat{f}^{\mu}(p) \frac{d^{3} p}{p_{0}}
\end{aligned}
$$


Now $A_{1}(f)$ is complex linear in $f$, hence is not Krein-symmetric if $f$ is not real, and it produces a complex-valued symplectic form:

$$
\left[A_{1}(\hat{f}), A_{1}(\hat{h})\right]=i \iint f_{\mu}(x) h^{\mu}(y) D(x-y) d^{4} x d^{4} y
$$

hence it is not possible to define a CCR-algebra with this form. It is causal though.

The choice which we use in this paper, is $A_{2}(f)$, and the reason for this is that it is the smearing which was necessary to define our gauge transformations Eqn (8). Furthermore, $A_{2}(f)$ is always Krein symmetric (and real linear), and it defines a real valued symplectic form

$$
\left[A_{2}(\hat{f}), A_{2}(\hat{h})\right]=i D(\hat{f}, \hat{h})=i \operatorname{Im} K(\hat{f}, \hat{h})
$$

which we can (and did) use to define a CCR-algebra. $D$ is not causal for complex-valued functions, but we have compensated for this by only extending the real space $\mathfrak{X}$ by complex multiples of gradients $\mathfrak{G}$. These gradients will be eliminated by the subsequent constrainings below. Their purpose is to select the physical subalgebras.

\subsection{Reduction isotony and weak causality.}

In this subsection we establish reduction isotony and weak causality for our example in Theorems 5.6 and 5.7. We first enforce the T-procedure locally as in Section 3, to obtain the objects:

$$
\begin{aligned}
\mathfrak{S}_{D}^{\Theta} & :=\left\{\omega \in \mathfrak{S}(\mathcal{F}(\Theta)) \mid \omega\left(U_{T_{h}}\right)=1 \quad \forall h \in \mathcal{S}\left(\mathbb{R}^{4}, \mathbb{R}\right), \quad \operatorname{supp}(h) \subset \Theta\right\} \\
\mathcal{D}(\Theta) & :=[\mathcal{F}(\Theta)(\mathcal{U}(\Theta)-\mathbb{1})] \cap[(\mathcal{U}(\Theta)-\mathbb{1}) \mathcal{F}(\Theta)], \\
\mathcal{O}(\Theta) & :=\{F \in \mathcal{F}(\Theta) \mid F D-D F \in \mathcal{D}(\Theta) \forall D \in \mathcal{D}(\Theta)\}=M_{\mathcal{F}(\Theta)}(\mathcal{D}(\Theta)), \\
\mathcal{R}(\Theta) & :=\mathcal{O}(\Theta) / \mathcal{D}(\Theta) \quad \text { where } \Theta \text { is any open set in } \mathbb{R}^{4} .
\end{aligned}
$$

For reduction isotony we need to prove that if $\Theta_{1} \subseteq \Theta_{2}$ then $\mathcal{D}\left(\Theta_{1}\right)=\mathcal{D}\left(\Theta_{2}\right) \cap \mathcal{O}\left(\Theta_{1}\right)$ and $\mathcal{O}\left(\Theta_{1}\right) \subseteq \mathcal{O}\left(\Theta_{2}\right)$, and this requires more explicit characterization of the local algebras involved.

\subsection{Theorem We have:}

$$
\begin{aligned}
\mathcal{O}(\Theta) & =\mathrm{C}^{*}\left(\delta_{\mathfrak{p}(\Theta)} \cup \mathcal{D}(\Theta)\right)=\left[\delta_{\mathfrak{p}(\Theta)} \cup \mathcal{D}(\Theta)\right]=\mathrm{C}^{*}\left(\delta_{\mathfrak{p}(\Theta)}\right)+\mathcal{D}(\Theta) \text { where } \\
\mathfrak{p}(\Theta) & :=\left\{f \in \mathfrak{Z}(\Theta) \mid T_{h}(f)=f \forall h \in \mathcal{S}\left(\mathbb{R}^{4}, \mathbb{R}\right), \quad \operatorname{supp}(h) \subset \Theta\right\} \\
& =\left\{f \in \mathfrak{Z}(\Theta) \mid B\left(f, G_{h}(f)\right)=0 \forall h \in \mathcal{S}\left(\mathbb{R}^{4}, \mathbb{R}\right), \quad \operatorname{supp}(h) \subset \Theta\right\} \\
& =\left\{f \in \mathfrak{Z}(\Theta)\left|p_{\mu} f^{\mu}\right| C_{+}=0\right\}
\end{aligned}
$$

with $G_{h}(f):=T_{h}(f)-f$. Moreover $\mathcal{R}(\Theta) \cong \mathrm{C}^{*}\left(\delta_{\mathfrak{p}(\Theta)}\right)$.

Proof: For any $f \in \mathfrak{p}(\Theta)$ one has $\delta_{f}=\beta_{T_{h}}\left(\delta_{f}\right)=U_{T_{h}} \delta_{f} U_{T_{h}}^{*}, \operatorname{supp}(h) \subset \Theta$, so that $\delta_{\mathfrak{p}(\Theta)} \subset$ $\mathcal{U}(\Theta)^{\prime}$ and Theorem $2.3(\mathrm{v})$ implies $\delta_{\mathfrak{p}(\Theta)} \subset \mathcal{O}(\Theta)$. Further $\mathcal{D}(\Theta) \subset \mathcal{O}(\Theta)$ proves the inclusion $\mathrm{C}^{*}\left(\delta_{\mathfrak{p}(\Theta)} \cup \mathcal{D}(\Theta)\right) \subseteq \mathcal{O}(\Theta)$. To show the reverse inclusion take $A \in \mathcal{O}(\Theta) \subset \mathcal{F}(\Theta)$ and from Lemma 5.2 there is a sequence $\left\{A_{n}\right\}_{n \in \mathbb{N}} \subset \operatorname{span}\left\{\delta_{\mathfrak{Z}(\Theta)} U_{\mathcal{G}(\Theta)}\right\}$ converging in the $\mathrm{C}^{*}$-norm to $A$. Put $A_{n}:=\sum_{i=1}^{k_{n}} \lambda_{i}^{n} \delta_{f_{i}^{n}} U_{\gamma_{i}^{n}}, \lambda_{i}^{n} \in \mathbb{C}, f_{i}^{n} \in \mathfrak{Z}(\Theta), \gamma_{i}^{n} \in \mathcal{G}(\Theta)$, and since $\left\{f_{i}^{n} \mid i=1, \ldots, k_{n}, n \in \mathbb{N}\right\}$ is a denumerable set we can rearrange it into a single sequence $\left\{f_{i}\right\}_{i \in \mathbb{N}}$, where $f_{i} \neq f_{j}$ if $i \neq j$. Thus we can rewrite

$$
A_{n}=\sum_{i=1}^{N_{n}} \delta_{f_{i}} \sum_{j=1}^{L_{n}} \lambda_{i j}^{(n)} U_{\gamma_{i j}^{(n)}} .
$$


Observe that for $\omega \in \mathfrak{S}_{D}^{\Theta}$ we have $\pi_{\omega}\left(A_{n}\right) \Omega_{\omega}=\sum_{i} \zeta_{i}^{(n)} \pi_{\omega}\left(\delta_{f_{i}}\right) \Omega_{\omega}$ where $\zeta_{i}^{(n)}:=\sum_{j} \lambda_{i j}^{(n)}$. Now $A \in \mathcal{O}(\Theta)$ and therefore we have for any $\omega \in \mathfrak{S}_{D}^{\Theta}$,

$$
\begin{aligned}
0 & =\pi_{\omega}\left(\left(U_{T_{h}}-\mathbb{1}\right) \cdot A\right) \Omega_{\omega}=\lim _{n \rightarrow \infty} \pi_{\omega}\left(\left(U_{T_{h}}-\mathbb{1}\right) \cdot A_{n}\right) \Omega_{\omega} \\
& =\lim _{n \rightarrow \infty} \pi_{\omega}\left(\beta_{T_{h}}\left(A_{n}\right)-A_{n}\right) \Omega_{\omega}=\lim _{n \rightarrow \infty} \sum_{i=1}^{N_{n}} \zeta_{i}^{(n)} \pi_{\omega}\left(\delta_{T_{h}\left(f_{i}\right)}-\delta_{f_{i}}\right) \Omega_{\omega}
\end{aligned}
$$

for $h \in \mathcal{S}\left(\mathbb{R}^{4}, \mathbb{R}\right), \quad \operatorname{supp}(h) \subset \Theta$, where we made use of $(\mathcal{U}(\Theta)-\mathbb{1}) \mathcal{O}(\Theta) \subset \mathcal{D}(\Theta) \subset N_{\omega}$ at the start. In particular let $\omega$ be an extension of the central state $\omega_{0}$ defined in Eq. (4) (which has Dirac state extensions by Theorem 4.1). Then for all $h$ :

$$
\begin{aligned}
& 0= \omega\left(A^{*}\left(U_{T_{h}}-\mathbb{1}\right)^{*}\left(U_{T_{h}}-\mathbb{1}\right) A\right) \\
&= \lim _{n \rightarrow \infty}\left\{2 \omega\left(A_{n}^{*} A_{n}\right)\right. \\
&\left.\quad-2 \operatorname{Re}\left(\sum_{i, j}^{N_{n}} \bar{\zeta}_{i}^{(n)} \zeta_{j}^{(n)} \exp \left[i B\left(f_{j}, T_{h}\left(f_{i}\right)\right) / 2\right] \omega\left(\delta_{f_{j}-T_{h}\left(f_{i}\right)}\right)\right)\right\} \\
&=2 \lim _{n \rightarrow \infty}\left\{\sum_{i}^{N_{n}}\left|\zeta_{i}^{(n)}\right|^{2}-\operatorname{Re}\left(\sum_{(i, j) \in P_{h}(n)} \bar{\zeta}_{i}^{(n)} \zeta_{j}^{(n)}\right)\right\}
\end{aligned}
$$

where $P_{h}(n):=\left\{(i, j) \in\left\{1, \ldots, N_{n}\right\}^{2} \mid f_{j}=T_{h}\left(f_{i}\right)\right\} \subset P_{h}(n+m)$. Observe that if $f_{i} \in \mathfrak{p}(\Theta)$, then $(i, i) \in P_{h}(n)$ for all $h$, and that these terms cancel in Eqn. (10), i.e. we may assume that $f_{i} \notin \mathfrak{p}(\Theta)$ in $(10)$. Furthermore by the Cauchy-Schwarz inequality

$$
\left|\sum_{(i, j) \in P_{h}(n)} \bar{\zeta}_{i}^{(n)} \zeta_{j}^{(n)}\right| \leq\left(\sum_{i \in D_{h}(n)}\left|\zeta_{i}^{(n)}\right|^{2}\right)^{\frac{1}{2}}\left(\sum_{j \in R_{h}(n)}\left|\zeta_{j}^{(n)}\right|^{2}\right)^{\frac{1}{2}} \leq \sum_{i=1}^{N_{n}}\left|\zeta_{i}^{(n)}\right|^{2}
$$

where $D_{h}(n):=\left\{i \mid(i, j) \in P_{h}(n)\right\}$ and $R_{h}(n):=\left\{j \mid(i, j) \in P_{h}(n)\right\}$ i.e. the domain and range of the relation defined by $P_{h}(n)$. If $D_{h}(n)$ or $R_{h}(n)$ is not $\left\{1,2, \ldots, N_{n}\right\}$, then the last inequality is strict and Eqn. (10) cannot hold unless $\lim _{n \rightarrow \infty} \zeta_{i}^{(n)}=0$ for all $i \leq N_{n}$ not in $D_{h}(n)$ or $R_{h}(n)$. Given that $f_{i} \notin \mathfrak{p}(\Theta)$ in the surviving terms of the sum, for each $i$, choose an $h$ such that $f_{i} \neq T_{h}\left(f_{i}\right)$, then by Eqn. (8) $T_{t h}\left(f_{i}\right)=f_{i}+t^{2}\left(T_{h}\left(f_{i}\right)-f_{i}\right)$ for $t \in \mathbb{R}_{+}$, and so $\left\{T_{t h}\left(f_{i}\right) \mid t \in \mathbb{R}_{+}\right\}$ is a continuous family of distinct elements of $\mathfrak{Z}(\Theta)$. Since $\left\{f_{j} \mid j \in \mathbb{N}\right\}$ is denumerable, there exists a $t_{0}$ such that $T_{t_{0}}\left(f_{i}\right) \neq f_{j}$ for all $j \in \mathbb{N}$, i.e. $i \notin D_{t_{0} h}(n)$ for all $n$, and so Eqn. (10) can only hold if $\lim _{n \rightarrow \infty} \zeta_{i}^{(n)}=0$. We conclude that in the original expression for $A_{n}$, if $f_{i} \notin \mathfrak{p}(\Theta)$, then $\lim _{n \rightarrow \infty} \zeta_{i}^{(n)}=0$.

Recall by Remark 8.4 in Appendix 1 that the factorization map $\mathcal{O}(\Theta) \rightarrow \mathcal{R}(\Theta)$ is precisely the restriction of $\mathcal{O}(\Theta)$ in the universal representation $\pi_{u}$ to the subspace $\mathcal{H}_{u}^{(p)}:=\left\{\psi \in \mathcal{H}_{u} \mid\right.$ $\left.\pi_{u}(\mathcal{U}(\Theta)) \psi=\psi\right\}$, so if we can show that $\pi_{u}(\mathcal{O}(\Theta))\left\lceil\mathcal{H}_{u}^{(p)}=\pi_{u}\left(\mathrm{C}^{*}\left(\delta_{\mathfrak{p}(\Theta)}\right)\right) \uparrow \mathcal{H}_{u}^{(p)}\right.$, that suffices to prove that $\mathcal{O}(\Theta)=\mathrm{C}^{*}\left(\delta_{\mathfrak{p}(\Theta)}\right)+\mathcal{D}(\Theta)$. Let $\psi \in \mathcal{H}_{u}^{(p)}$, and $f_{j} \notin \mathfrak{p}(\Theta)$ :

$$
\begin{aligned}
\pi_{u}(A) \psi & =\lim _{n \rightarrow \infty} \pi_{u}\left(A_{n}\right) \psi=\lim _{n \rightarrow \infty} \sum_{i=1}^{N_{n}} \zeta_{i}^{(n)} \pi_{u}\left(\delta_{f_{i}}\right) \psi \\
& =\lim _{n \rightarrow \infty}\left(\sum_{i \neq j}^{N_{n}} \zeta_{i}^{(n)} \pi_{u}\left(\delta_{f_{i}}\right)+\zeta_{j}^{(n)} \pi_{u}\left(\delta_{f_{j}}\right)\right) \psi \\
& =\lim _{n \rightarrow \infty} \sum_{i \neq j}^{N_{n}} \zeta_{i}^{(n)} \pi_{u}\left(\delta_{f_{i}}\right) \psi
\end{aligned}
$$


so we can omit all contributions where $f_{j} \notin \mathfrak{p}(\Theta)$ from the sum:

$$
\pi_{u}(A) \psi=\lim _{n \rightarrow \infty} \sum_{f_{i} \in \mathfrak{p}(\Theta)}^{N_{n}} \zeta_{i}^{(n)} \pi_{u}\left(\delta_{f_{i}}\right) \psi \quad \forall \psi \in \mathcal{H}_{u}^{(p)} .
$$

The latter will be in $\pi_{u}\left(\mathrm{C}^{*}\left(\delta_{\mathfrak{p}(\Theta)}\right)\right) \psi$, providing we can show that $\lim _{n \rightarrow \infty} \sum_{f_{i} \in \mathfrak{p}(\Theta)}^{N_{n}} \zeta_{i}^{(n)} \delta_{f_{i}}$ converges in the $\mathrm{C}^{*}$-norm. This is easy to see, because from the convergence of $A_{n}$ in Eqn. (9), we get the convergence of the subseries

$$
\sum_{f_{i} \in \mathfrak{p}(\Theta)}^{N_{n}} \delta_{f_{i}} \sum_{j=1}^{L_{n}} \lambda_{i j}^{(n)} U_{\gamma_{i j}^{(n)}} \in \mathrm{C}^{*}\left(\delta_{\mathfrak{p}(\Theta)} \cup \mathcal{U}(\Theta)\right)
$$

and since $\delta_{\mathfrak{p}(\Theta)}$ commutes with $\mathcal{U}(\Theta)$ there is a ${ }^{*}$-homomorphism $\varphi: \mathrm{C}^{*}\left(\delta_{\mathfrak{p}(\Theta)} \cup \mathcal{U}(\Theta)\right) \rightarrow$ $\mathrm{C}^{*}\left(\delta_{\mathfrak{p}(\Theta)}\right)$ by $\varphi(\mathcal{U}(\Theta))=\mathbb{1}$ (just apply the T-procedure), hence the image of the preceding sequence converges, i.e. $\lim _{n \rightarrow \infty} \sum_{f_{i} \in \mathfrak{p}(\Theta)}^{N_{n}} \zeta_{i}^{(n)} \delta_{f_{i}}$ converges in the $\mathrm{C}^{*}$-norm. Thus $\pi_{u}(\mathcal{O}(\Theta)) \psi \subseteq$ $\pi_{u}\left(\mathrm{C}^{*}\left(\delta_{\mathfrak{p}(\Theta)}\right)\right) \psi$ for all $\psi \in \mathcal{H}_{u}^{(p)}$. Hence $\mathcal{O}(\Theta)=\mathrm{C}^{*}\left(\delta_{\mathfrak{p}(\Theta)}\right)+\mathcal{D}(\Theta)$, using $\delta_{\mathfrak{p}(\Theta)} \subset \mathcal{O}(\Theta)$, (cf. $\left.\left.\otimes\right]\right)$.

For the last two equivalent characterisations of $\mathfrak{p}(\Theta)$, observe first that if $T_{h}(f)=f$, then $B\left(f, G_{h}(f)\right)=B\left(f, T_{h}(f)-f\right)=0$ and conversely

$$
0=B\left(f, G_{h}(f)\right)=-2 \pi\left|\int_{C_{+}} \frac{d^{3} p}{p_{0}} p_{\mu} f^{\mu}(p) \overline{\widehat{h}(p)}\right|^{2}
$$

which by Eqn. (8) implies that $G_{h}(f)=0$. Choose $\widehat{h}=i p_{\mu} f^{\mu}$ (which is in the allowed class of functions) to see the equivalence with $p_{\mu} f^{\mu}\left\lceil C_{+}=0\right.$.

Finally, to prove that $\mathcal{R}(\Theta)=\left(\mathrm{C}^{*}\left(\delta_{\mathfrak{p}(\Theta)}\right)+\mathcal{D}(\Theta)\right) / \mathcal{D}(\Theta) \cong \mathrm{C}^{*}\left(\delta_{\mathfrak{p}(\Theta)}\right)$, it suffices to show that the ideal $\mathrm{C}^{*}\left(\delta_{\mathfrak{p}(\Theta)}\right) \cap \mathcal{D}(\Theta)=\{0\}$. Consider a sequence

$$
A_{n}=\sum_{i=1}^{N_{n}} \lambda_{i}^{(n)} \delta_{f_{i}} \in \Delta(\mathfrak{p}(\Theta), B), \quad \lambda_{i}^{(n)} \in \mathbb{C}, \quad \text { converging to } \quad A \in \mathcal{D}(\Theta)
$$

then we show that it converges to zero. Now $\delta_{f_{j}} \in \mathcal{O}(\Theta)$ for $f_{j} \in \mathfrak{p}(\Theta)$, so for $N_{n}>j$ we have

$$
\delta_{-f_{j}} \cdot A_{n}=\sum_{i \neq j}^{N_{n}} \lambda_{i}^{(n)} \delta_{f_{i}-f_{j}} \exp \left(i B\left(f_{i}, f_{j}\right) / 2\right)+\lambda_{j}^{(n)} \mathbb{1} \longrightarrow \delta_{-f_{j}} \cdot A \in \mathcal{D}(\Theta) .
$$

Since the central state $\omega_{0}$ (cf. Eqn. (画) extends to a Dirac state we have

$$
0=\omega_{0}\left(\delta_{-f_{j}} \cdot A\right)=\lim _{n \rightarrow \infty} \omega_{0}\left(\delta_{-f_{j}} A_{n}\right)=\lim _{n \rightarrow \infty} \lambda_{j}^{(n)} \quad \forall j .
$$

This implies that $A=0$, because we can realize $A$ as an $\ell^{2}$-sequence over $\mathfrak{p}(\Theta)$ (recall discussion in Section 4.1), and the evaluation map at a point in $\mathfrak{p}(\Theta)$ is $\ell^{2}$-continuous, hence $\mathrm{C}^{*}$-continuous so by the previous equation evaluation of $A$ at each point is zero.

One can now set $\Theta=\mathbb{R}^{4}$ to get the global version of this theorem. An important physical observation, is that $\mathfrak{p}=\cup \mathfrak{p}(\Theta)$ contains the functions corresponding to the field operators $F_{\mu \nu}$. To see this, smear $F_{\mu \nu}(p)$ with an antisymmetric tensor function $f_{\mu \nu}$ to obtain $F(f)$, and note that the latter corresponds to the smearing of $A_{\mu}$ with $2 p_{\nu} f^{\mu \nu} \in \mathfrak{p}$.

5.6 Theorem The system of local constraints defined here satisfies reduction isotony. 
Proof: Let $\Theta_{1} \subseteq \Theta_{2}$, then we start by showing that $\mathcal{O}\left(\Theta_{1}\right) \subseteq \mathcal{O}\left(\Theta_{2}\right)$, i.e. by Theorem 5 .5 we show that $\mathrm{C}^{*}\left(\delta_{\mathfrak{p}\left(\Theta_{1}\right)}\right)+\mathcal{D}\left(\Theta_{1}\right) \subseteq \mathrm{C}^{*}\left(\delta_{\mathfrak{p}\left(\Theta_{2}\right)}\right)+\mathcal{D}\left(\Theta_{2}\right)$. This follows directly from $\mathcal{D}\left(\Theta_{1}\right) \subseteq \mathcal{D}\left(\Theta_{2}\right)$ and $\mathfrak{p}\left(\Theta_{1}\right) \subseteq \mathfrak{p}\left(\Theta_{2}\right)$ where the last inclusion comes from the last characterisation of $\mathfrak{p}(\Theta)$ in Theorem 5.5.

It only remains to show that $\mathcal{D}\left(\Theta_{1}\right)=\mathcal{D}\left(\Theta_{2}\right) \cap \mathcal{O}\left(\Theta_{1}\right)$. Recall that $\mathcal{D}(\Theta) \subset \mathcal{O}(\Theta)$, and that $\mathcal{D}(\Theta)$ is the largest $\mathrm{C}^{*}$-algebra in $\mathcal{F}(\Theta)$ (hence in $\mathcal{O}(\Theta)$ ) which is annihilated by all $\omega \in \mathfrak{S}_{D}^{\Theta}$. Since $\mathcal{O}\left(\Theta_{1}\right) \subseteq \mathcal{O}\left(\Theta_{2}\right)$, it suffices to show by Lemma 3.8 that every Dirac state on $\mathcal{O}\left(\Theta_{1}\right)$ extends to a Dirac state on $\mathcal{O}\left(\Theta_{2}\right)$.

Recall that $\mathcal{O}\left(\Theta_{1}\right)=\mathrm{C}^{*}\left(\delta_{\mathfrak{p}\left(\Theta_{1}\right)}\right)+\mathcal{D}\left(\Theta_{1}\right)$, so a Dirac state on $\mathcal{O}\left(\Theta_{1}\right)$ is uniquely determined by its values on $\delta_{\mathfrak{p}\left(\Theta_{1}\right)}$. Moreover, from the fact that $f \in \mathfrak{p}\left(\Theta_{1}\right)$ implies $p_{\mu} f^{\mu}{ }^{\uparrow} C_{+}=0$ and Eqn. (8), we see that $\mathcal{U}\left(\Theta_{2}\right)$ commutes with $\mathrm{C}^{*}\left(\delta_{\mathfrak{p}\left(\Theta_{1}\right)}\right)$ :

$$
\begin{aligned}
U_{T_{h}} \delta_{f} U_{T_{h}}^{-1} & =\delta_{T_{h} f}=\delta_{f} \quad \text { since } \\
\left(T_{h} f\right)_{\mu}(p) & =f_{\mu}(p)-i \pi p_{\mu} \widehat{h}(p) \int_{C_{+}} f^{\nu}\left(p^{\prime}\right) p_{\nu}^{\prime} \widehat{\widehat{h}\left(p^{\prime}\right)} \frac{d^{3} p^{\prime}}{p_{0}^{\prime}}=f_{\mu}(p)
\end{aligned}
$$

for $f \in \mathfrak{p}\left(\Theta_{1}\right)$. Next define $\widetilde{\mathcal{O}}:=\mathrm{C}^{*}\left(\delta_{\mathfrak{p}\left(\Theta_{1}\right)} \cup \mathcal{U}\left(\Theta_{2}\right)\right) \subset \mathcal{O}\left(\Theta_{2}\right)$. Now $\widetilde{\mathcal{O}}$ is generated by the two mutually commuting $\mathrm{C}^{*}$-algebras $\mathrm{C}^{*}\left(\delta_{\mathfrak{p}\left(\Theta_{1}\right)}\right)$ and $\mathrm{C}^{*}\left(\mathcal{U}\left(\Theta_{2}\right)\right) \cong \mathrm{C}^{*}\left(\mathcal{G}\left(\Theta_{2}\right)\right)$ where the latter is Abelian. If $A B=0$ for $A \in \mathrm{C}^{*}\left(\delta_{\mathfrak{p}\left(\Theta_{1}\right)}\right)$ and $B \in \mathrm{C}^{*}\left(\mathcal{U}\left(\Theta_{2}\right)\right)$, then either $A=0$ or $B=0$. This we can see from the realisation of $\mathrm{C}^{*}\left(\mathcal{U}\left(\Theta_{2}\right)\right)$ as scalar valued functions of denumerable support in $\mathcal{G}\left(\Theta_{2}\right)$, so (pointwise) multiplication by a nonzero $A \in \mathrm{C}^{*}\left(\delta_{\mathfrak{p}\left(\Theta_{1}\right)}\right)$ cannot change support. Then by an application of the result in [28, Exercise 2, p. 220], we conclude that the map $\varphi(A \otimes B):=A B$, $A \in \mathrm{C}^{*}\left(\delta_{\mathfrak{p}\left(\Theta_{1}\right)}\right), B \in \mathrm{C}^{*}\left(\mathcal{U}\left(\Theta_{2}\right)\right)$ extends to an isomorphism $\varphi: \mathrm{C}^{*}\left(\delta_{\mathfrak{p}\left(\Theta_{1}\right)}\right) \otimes \mathrm{C}^{*}\left(\mathcal{U}\left(\Theta_{2}\right)\right) \rightarrow \widetilde{\mathcal{O}}$. (Note that since $\mathrm{C}^{*}\left(\mathcal{U}\left(\Theta_{2}\right)\right)$ is commutative it is nuclear, hence the tensor norm is unique).

Let $\omega \in \mathfrak{S}_{D}^{\Theta_{1}} \uparrow \mathcal{O}\left(\Theta_{1}\right)$ and define a product state $\widetilde{\omega}$ on $\widetilde{\mathcal{O}}$ by $\widetilde{\omega}:=\omega \otimes \hat{\omega}$, where $\hat{\omega}$ is the state $\hat{\omega}\left(U_{\theta}\right)=1$ for all $\theta \in \mathcal{G}\left(\Theta_{2}\right)$. Now extend $\widetilde{\omega}$ arbitrarily to $\mathcal{O}\left(\Theta_{2}\right)$, then since it coincides with $\omega$ on $\delta_{\mathfrak{p}\left(\Theta_{1}\right)}$ and $\widetilde{\omega}\left(\mathcal{U}\left(\Theta_{2}\right)\right)=1$, it is a Dirac state on $\mathcal{O}\left(\Theta_{2}\right)$ which extends $\omega\left\lceil\mathcal{O}\left(\Theta_{1}\right)\right.$.

5.7 Theorem The system of local quantum constraints $(\mathcal{F}(\Theta), \mathcal{U}(\Theta))$ satisfies weak causality, i.e. if $\Theta_{1} \perp \Theta_{2}$ then $\left[\mathcal{O}\left(\Theta_{1}\right), \mathcal{O}\left(\Theta_{2}\right)\right] \subset \mathcal{D}\left(\Theta_{0}\right)$ for some $\Theta_{0} \supset \Theta_{1} \cup \Theta_{2}, \Theta_{i} \in \Gamma$.

Proof: Since $\mathcal{O}(\Theta)=\mathrm{C}^{*}\left(\delta_{\mathfrak{p}(\Theta)}\right)+\mathcal{D}(\Theta)$ it is sufficient to consider commutants of $A_{i} \in \mathcal{O}\left(\Theta_{i}\right)$ being generating elements: $A_{i}=\delta_{f_{i}}+D_{i}$ for $f_{i} \in \mathfrak{p}\left(\Theta_{i}\right), D_{i} \in \mathcal{D}\left(\Theta_{i}\right), i=1,2$. Now

$$
\begin{aligned}
{\left[A_{1}, A_{2}\right]=} & {\left[\delta_{f_{1}}, \delta_{f_{2}}\right]+\left[\delta_{f_{1}}, D_{2}\right]+\left[D_{1}, \delta_{f_{2}}\right]+\left[D_{1}, D_{2}\right] } \\
= & \left(e^{\frac{i}{2} B\left(f_{1}, f_{2}\right)}-e^{-\frac{i}{2} B\left(f_{1}, f_{2}\right)}\right) \delta_{f_{1}+f_{2}} \\
& +\left[\delta_{f_{1}}, D_{2}\right]+\left[D_{1}, \delta_{f_{2}}\right]+\left[D_{1}, D_{2}\right]
\end{aligned}
$$

The first term vanishes because $\Theta_{1} \perp \Theta_{2}$ implies the supports of $f_{1}$ and $f_{2}$ are spacelike separated, so

$$
B\left(\widehat{f}_{1}, \widehat{f}_{2}\right)=\int d x d x^{\prime} D\left(x-x^{\prime}\right) f_{1}^{\mu}(x) f_{2 \mu}\left(x^{\prime}\right)=0
$$

because the Pauli-Jordan distribution $D$ has support inside the closed forward and backward light cones [29, p. 214]. Further for any $\Theta_{0} \supset \Theta_{1} \cup \Theta_{2}$ reduction isotony implies $\mathcal{D}\left(\Theta_{1}\right) \subset \mathcal{D}\left(\Theta_{0}\right) \supset$ $\mathcal{D}\left(\Theta_{2}\right)$ and $\mathcal{O}\left(\Theta_{1}\right) \subset \mathcal{O}\left(\Theta_{0}\right) \supset \mathcal{O}\left(\Theta_{2}\right)$. But $\mathcal{D}\left(\Theta_{0}\right)$ is a closed 2-sided ideal in $\mathcal{O}\left(\Theta_{0}\right)$ and therefore the last 3 terms of the sum above are contained in $\mathcal{D}\left(\Theta_{0}\right)$ and the proof is concluded.

5.8 Remark Note that the net $\Theta \rightarrow \mathcal{F}(\Theta)$ does not satisfy the causality property, as we expect from the choice of noncausal constraints $\left(\partial^{\mu} A_{\mu}\right)^{(+)}(x)$. To see this, let $\Theta_{1} \perp \Theta_{2}$, and let $\delta_{f} \in$ 
$\mathcal{F}\left(\Theta_{1}\right)$ and $U_{T_{h}} \in \mathcal{F}\left(\Theta_{2}\right)$, then the commutator $\left[\delta_{f}, U_{T_{h}}\right]$ need not vanish because in Eqn. (8) we can have that

$$
c(f, h)=\int_{C_{+}} \widehat{f}^{\nu}(p) p_{\nu} \overline{\widehat{h}(p)} \frac{d^{3} p}{p_{0}} \neq 0
$$

for $\operatorname{supp}(f) \subset \Theta_{1}$ and $\operatorname{supp}(h) \subset \Theta_{2}$.

\subsection{Covariance.}

In order to examine weak covariance for this system of local constraints, we first need to define the action of $\mathcal{P}_{+}^{\uparrow}$ on $\mathcal{F}_{e}$. We start with the usual action of $\mathcal{P}_{+}^{\uparrow}$ on $\mathcal{S}\left(\mathbb{R}^{4}, \mathbb{C}^{4}\right)$. Define

$$
\left(V_{g} f\right)(p):=e^{-i p a} \Lambda f\left(\Lambda^{-1} p\right) \quad \forall f \in \mathcal{S}\left(\mathbb{R}^{4}, \mathbb{C}^{4}\right), g=(\Lambda, a) \in \mathcal{P}_{+}^{\uparrow} .
$$

Then $V_{g}$ is symplectic, hence factors through to a symplectic transformation on $\mathfrak{Y}$, and this defines an action $\alpha: \mathcal{P}_{+}^{\uparrow} \rightarrow \operatorname{Aut}(\overline{\Delta(\mathfrak{Y}, B)})$ by $\alpha_{g}\left(\delta_{f}\right):=\delta_{V_{g} f}, f \in \mathfrak{Y}$.

5.9 Lemma Define $\alpha_{g}\left(U_{T_{h}}\right):=U_{T_{W_{g}}}$, where $\left(\widehat{W_{g} h}\right)(p):=e^{-i a \cdot p} \widehat{h}\left(\Lambda^{-1} p\right)$, and we chose $g=$ $(\Lambda, a) \in \mathcal{P}_{+}^{\uparrow}$. Then this extends $\alpha_{g}$ from $\overline{\Delta(\mathfrak{Y}, B)}$ to $\mathcal{F}_{e}$, producing a consistent action $\alpha: \mathcal{P}_{+}^{\uparrow} \rightarrow$ $\operatorname{Aut}\left(\mathcal{F}_{e}\right)$.

Proof: We need to show that if we extend $\alpha_{g}$ from the set $\overline{\Delta(\mathfrak{Y}, B)} \cup\left\{U_{T_{h}} \mid h\right\}$ to the *-algebra generated by it using the homomorphism property of $\alpha_{g}$, then this is consistent with all relations of the $U_{T_{h}}$ amongst themselves, and between them and $\overline{\Delta(\mathfrak{Y}, B)}$. First we need to establish how $V_{g}$ and $T_{h}$ intertwines.

$$
\begin{aligned}
\left(T_{W_{g} h} V_{g} f\right)_{\mu}(p) & =\left(V_{g} f\right)_{\mu}(p)-i \pi p_{\mu} \widehat{W_{g} h}(p) \int_{C_{+}} \frac{d^{3} p^{\prime}}{p_{0}^{\prime}}\left(V_{g} f\right)^{\nu}\left(p^{\prime}\right) p_{\nu}^{\prime} \overline{\widehat{W_{g} h}\left(p^{\prime}\right)} \\
& =\left(V_{g} f\right)_{\mu}(p)-i \pi p_{\mu} e^{-i p \cdot a} \widehat{h}\left(\Lambda^{-1} p\right) \int_{C_{+}} \frac{d^{3} p^{\prime}}{p_{0}^{\prime}}(\Lambda f)^{\nu}\left(\Lambda^{-1} p^{\prime}\right) p_{\nu}^{\prime} \overline{\widehat{h}\left(p^{\prime}\right)} \\
& =\left(V_{g} T_{h} f\right)_{\mu}(p) .
\end{aligned}
$$

Thus $V_{g} T_{h}=T_{W_{g} h} V_{g}$. Now the basic relation between $\overline{\Delta(\mathfrak{Y}, B)}$ and $\left\{U_{T_{h}} \mid h\right\}$ is the implementing relation, so

$$
\begin{aligned}
\alpha_{g}\left(U_{T_{h}} \delta_{f} U_{T_{h}}^{*}\right) & =\alpha_{g}\left(\delta_{T_{h} f}\right)=\delta_{V_{g} T_{h} f}=\delta_{T_{W_{g} h} V_{g} f} \\
& =U_{T_{W_{g} h}} \delta_{V_{g} f} U_{T_{W_{g} h}^{*}}^{*}=\alpha_{g}\left(U_{T_{h}}\right) \alpha_{g}\left(\delta_{f}\right) \alpha_{g}\left(U_{T_{h}}\right)^{*}
\end{aligned}
$$

thus $\alpha_{g}$ is consistent with this. Finally we need to show that $\alpha_{g}$ respects any group identities in $\beta(\mathcal{G}) \subset$ Aut $\overline{\Delta(\mathfrak{Y}, B)}$. Recalling that $\mathcal{G}$ consists of finite products of $T_{h}$, let $\gamma=T_{h_{1}} \ldots T_{h_{n}} \in \mathcal{G}$, then $\gamma \rightarrow T_{W_{g} h_{1}} \ldots T_{W_{g} h_{n}}$ defines a consistent group homomorphism because

$$
\begin{aligned}
\alpha_{g}\left(\beta_{\gamma}\left(\delta_{f}\right)\right) & =\alpha_{g}\left(\delta_{T_{h_{1}} \cdots T_{h_{n}} f}\right)=\delta_{V_{g} T_{h_{1}} \ldots T_{h_{n}} f} \\
& =\delta_{T_{W_{g} h_{1}} \cdots T_{W_{g} h_{n}} V_{g} f}=\beta\left(T_{W_{g} h_{1}} \cdots T_{W_{g} h_{n}}\right) \alpha_{g}\left(\delta_{f}\right)
\end{aligned}
$$

i.e. $\beta\left(T_{W_{g} h_{1}} \cdots T_{W_{g} h_{n}}\right)=\alpha_{g} \circ \beta_{\gamma} \circ \alpha_{g}^{-1}$. Thus $\alpha_{g}\left(U_{T_{h}}\right)=U_{T_{W_{g} h}}$ extends consistently to $U_{\mathcal{G}}$.

Observe that the action $V_{g}$ preserves the reality condition $\overline{f(p)}=f(-p)$ which defines $\mathfrak{X}$, hence it preserves $\mathfrak{X}$ and in fact $V_{g} \mathfrak{X}(\Theta)=\mathfrak{X}(g \Theta)$.

5.10 Theorem Consider the action $\alpha: \mathcal{P}_{+}^{\uparrow} \rightarrow$ Aut $\mathcal{F}_{e}$ defined above. Then the system of local quantum constraints $\Gamma \ni \Theta \rightarrow(\mathcal{F}(\Theta), \mathcal{U}(\Theta))$ satisfies $\alpha_{g}(\mathcal{U}(\Theta))=\mathcal{U}(g \Theta)$ and the net $\Gamma \ni \Theta \rightarrow$ $\mathcal{F}(\Theta)$ transforms covariantly, i.e. $\alpha_{g}(\mathcal{F}(\Theta))=\mathcal{F}(g \Theta), \Theta \in \Gamma$. Therefore the local observables define a covariant net, i.e. $\alpha_{g}(\mathcal{O}(\Theta))=\mathcal{O}(g \Theta)$. 
Proof: We have:

$$
\begin{aligned}
\alpha_{g}(\mathcal{U}(\Theta)) & =\alpha_{g}\left(\left\{U_{T_{h}} \mid \operatorname{supp}(h) \subset \Theta\right\}\right)=\left\{U_{T_{V_{g}}} \mid \operatorname{supp}(h) \subset \Theta\right\} \\
& \subseteq\left\{U_{T_{h}} \mid \operatorname{supp}(h) \subset g \Theta\right\}=\mathcal{U}(g \Theta)
\end{aligned}
$$

and replacing $g$ by $g^{-1}$ gives the reverse inclusion.

For covariance of the net $\mathcal{F}(\Theta)$, recall that each $\mathcal{F}(\Theta)$ is generated by $\mathcal{U}(\Theta)$ and $\delta_{\mathfrak{X}(\Theta)}$, so since

$$
\alpha_{g}\left(\delta_{\mathfrak{X}(\Theta)}\right)=\delta_{V_{g} \mathfrak{X}(\Theta)}=\delta_{\mathfrak{X}(g \Theta)},
$$

it follows that $\alpha_{g}(\mathcal{F}(\Theta))=\mathcal{F}(g \Theta)$. The covariance property for the net of local observables follows from Remark 3.23 (i)

Finally putting together Theorems 5.6, 5.7, 5.10, 3.25 we have proved for the Gupta-Bleuler model a major claim:

5.11 Theorem The system of local quantum constraints $\Gamma \ni \Theta \rightarrow(\mathcal{F}(\Theta), \mathcal{U}(\Theta))$ satisfies reduction isotony, weak causality and covariance and therefore the corresponding net of local physical observables $\Gamma \ni \Theta \rightarrow \mathcal{R}(\Theta)$ is a $H K-Q F T$.

\subsection{A simple physical observable algebra}

The net $\Theta \rightarrow \mathcal{R}(\Theta)=\mathrm{C}^{*}\left(\delta_{\mathfrak{p}(\Theta)}\right)$ produces a quasi-local physical algebra

$$
\mathcal{R}_{0}=\underset{\lim }{\longrightarrow} \mathcal{R}(\Theta)=\lim _{\longrightarrow} \mathrm{C}^{*}\left(\delta_{\mathfrak{p}(\Theta)}\right)=\mathrm{C}^{*}\left(\delta_{\mathfrak{p}}\right)=\overline{\Delta(\mathfrak{p}, B)},
$$

where $\mathfrak{p}:=\operatorname{Span}\{\mathfrak{p}(\Theta) \mid \Theta \in \Gamma\}=\bigcup_{\Theta \in \Gamma} \mathfrak{p}(\Theta)$ since $\Gamma$ is a directed set. Since $B$ is degenerate on $\mathfrak{p}$ (see below), $\mathcal{R}_{0}$ is not simple and thus cannot be the final physical algebra. This is also evident from the fact that $\mathfrak{p}$ contains complex multiples of gradients, so $\mathfrak{p}$ is not in $\mathfrak{X}$. Moreover since we have not enforced Maxwell's equations, from a physical point of view $\mathcal{R}_{0}$ cannot be considered as representing the observables of an electromagnetic field as yet. To solve these problems, we now do a second stage of constraining where we choose for our constraint system $\left(\mathcal{R}_{0}, \widetilde{\mathcal{U}}\right)$ where $\widetilde{\mathcal{U}}:=\delta_{\mathfrak{p}_{0}}$ and $\mathfrak{p}_{0}$ is the kernel of $B \mid \mathfrak{p}$. The T-procedure applied to this pair will result in a simple algebra via Corollary 5.4 in [8]. For the connection with the Maxwell equations, we need the following proposition:

\subsection{Proposition We have:}

$$
\begin{aligned}
\mathfrak{p}_{0}:= & \{f \in \mathfrak{p} \mid B(f, k)=0 \quad \forall k \in \mathfrak{p}\} \\
= & \left\{f \in \mathfrak{p} \mid f_{\mu}(p)=p_{\mu} h(p) \text { for } p \in C_{+}, \text {where } h: C_{+} \rightarrow \mathbb{C}\right. \\
& \left.\quad \text { is any function such that } p \rightarrow p_{\mu} h(p) \text { is in } \mathfrak{Z}_{(0)}\right\}
\end{aligned}
$$

where $\mathfrak{Z}_{(0)}=\mathfrak{Z} \cap C_{c}^{\infty}\left(\mathbb{R}^{4}, \mathbb{C}^{4} \widehat{)} / \operatorname{Ker}(D)\right.$.

Proof: Recall that $\mathfrak{Z}=\mathfrak{X}+\mathbb{C} \cdot \mathfrak{G}$, then it is easy to see from Theorem 5.5 that the gradients $\mathbb{C} \cdot \mathfrak{G} \cap \mathfrak{Z}_{(0)}$ are in $\mathfrak{p}$. Moreover, we have in fact that $\mathbb{C} \cdot \mathfrak{G} \cap \mathfrak{Z}_{(0)} \subset \operatorname{Ker}(B \mid \mathfrak{p})$ since if we take $h_{\mu}=p_{\mu} k \in \mathbb{C} \cdot \mathfrak{G}$ and $f \in \mathfrak{p}$ (hence $p_{\mu} f^{\mu}=0$ ), then

$$
B(h, f)=i \pi \int_{C_{+}}\left(f_{\mu}(p) p^{\mu} \overline{k(p)}-\overline{f_{\mu}(p)} p^{\mu} k(p)\right) \frac{d^{3} p}{p_{0}}=0 .
$$

Thus $\mathfrak{p}_{0}=\mathbb{C} \cdot \mathfrak{G} \cap \mathfrak{Z}_{(0)}+\operatorname{Ker}(B\lceil(\mathfrak{p} \cap \mathfrak{X}))$. Now, to examine $\operatorname{Ker}(B\lceil(\mathfrak{p} \cap \mathfrak{X}))$ we first want to extend to a larger class of functions, since $\mathfrak{p}$ consists of Fourier transforms of functions of compact support, 
hence cannot have compact support, which we will want to use below. Now $\mathfrak{p} \cap \mathfrak{X} \subset \mathfrak{X}_{(0)}=$ $\rho\left(C_{c}^{\infty}\left(\mathbb{R}^{4}, \mathbb{R}^{4}\right)\right)$ where $\rho(f):=\widehat{f} \mid C_{+}$and by definition $D(\widehat{f}, \widehat{k})=: B(\rho(f), \rho(k))$. Moreover, by Theorem 5.5, $\mathfrak{p} \cap \mathfrak{X}=\rho(\mathfrak{P})$ where $\mathfrak{P}:=\left\{f \in C_{c}^{\infty}\left(\mathbb{R}^{4}, \mathbb{R}^{4}\right) \mid \partial^{\mu} f_{\mu}=0\right\}$. Since the smooth functions of compact support are dense with respect to the Schwartz topology in the Schwartz space, and the divergence operator is continuous for the Schwartz topology, the closure of $\mathfrak{P}$ in the Schwartz topology is $\widetilde{\mathfrak{P}}:=\left\{f \in \mathcal{S}\left(\mathbb{R}^{4}, \mathbb{R}^{4}\right) \mid \partial^{\mu} f_{\mu}=0\right\}$. It is well-known that $\widehat{D}$ is a tempered distribution (it is the two-point function for the free electromagnetic field), hence it is continuous with respect to the Schwartz topology on $\mathcal{S}\left(\mathbb{R}^{4}, \mathbb{R}^{4}\right)$ in each entry. Thus $\widehat{D}(f, k)=0$ for all $k \in \mathfrak{P}$ iff $\widehat{D}(f, k)=0$ for all $k \in \widetilde{\mathfrak{P}}$ and hence $\operatorname{Ker}(B \mid \mathfrak{p} \cap \mathfrak{X})=\{f \in \mathfrak{p} \cap \mathfrak{X} \mid B(f, k)=0 \quad \forall k \in \widetilde{\mathfrak{p}}\}$ where $\widetilde{\mathfrak{p}}:=\rho(\widetilde{\mathfrak{P}})$. We will need this below.

Let $f \in \mathfrak{p} \cap \mathfrak{X}$, so $p_{\mu} f^{\mu}(p)=0$ for $p \in C_{+}$, i.e. $\mathbf{p} \cdot \mathbf{f}(p)=\|\mathbf{p}\| f_{0}(p)$, so for $p \in C_{+} \backslash 0$, we have $f_{0}(p)=\mathbf{p} \cdot \mathbf{f}(p) /\|\mathbf{p}\|=\mathbf{e}(p) \cdot \mathbf{f}(p)$ where $\mathbf{e}(p):=\mathbf{p} /\|\mathbf{p}\|$. Now in terms of real and imaginary parts $f=u+i v \in \operatorname{Ker}(B \mid \mathfrak{p} \cap \mathfrak{X})$ iff for all $k=w+i r \in \widetilde{\mathfrak{p}}$ we have that

$$
\begin{aligned}
0 & =D(f, k)=2 i \int_{\mathbb{R}^{3} \backslash 0}\left(v_{\mu} w^{\mu}-u_{\mu} r^{\mu}\right) \frac{d^{3} p}{\|\mathbf{p}\|} \quad \text { (using Eqn. (7) ) } \\
& =2 i \int_{\mathbb{R}^{3} \backslash 0}(\mathbf{u} \cdot \mathbf{r}-\mathbf{v} \cdot \mathbf{w}+(\mathbf{e}(p) \cdot \mathbf{v})(\mathbf{e}(p) \cdot \mathbf{w})-(\mathbf{e}(p) \cdot \mathbf{u})(\mathbf{e}(p) \cdot \mathbf{r})) \frac{d^{3} p}{\|\mathbf{p}\|}
\end{aligned}
$$

Choose $w=0$ (which is possible in $\widetilde{\mathfrak{p}})$ to get that for all $r \in \widetilde{\mathfrak{p}} \cap\left(\mathcal{S}_{-}\left\lceil C_{+}\right)\right.$(recall Remark 5.1):

$$
\begin{aligned}
0 & =D(f, k)=2 i \int_{\mathbb{R}^{3} \backslash 0}(\mathbf{u} \cdot \mathbf{r}-(\mathbf{e}(p) \cdot \mathbf{u})(\mathbf{e}(p) \cdot \mathbf{r})) \frac{d^{3} p}{\|\mathbf{p}\|} \\
& =2 i \int_{\mathbb{R}^{3} \backslash 0} \mathbf{r} \cdot(\mathbf{u}-\mathbf{e}(p)(\mathbf{e}(p) \cdot \mathbf{u})) \frac{d^{3} p}{\|\mathbf{p}\|} .
\end{aligned}
$$

Now let $m: C_{+} \rightarrow \mathbb{R}_{+}$be a smooth bump function with compact support away from zero, then we know that that the function $s$ given by

$$
\mathbf{s}(p):=(\mathbf{u}(p)-\mathbf{e}(p)(\mathbf{e}(p) \cdot \mathbf{u}(p))) m(p) \quad \text { and } \quad s_{0}(p):=\mathbf{e}(p) \cdot \mathbf{s}(p)
$$

is in $\mathfrak{X}$ by the characterisation of $\mathfrak{X}$ given in Remark 5.1(i), that it contains all smooth functions with compact support away from zero. Moreover, since $p_{\mu} s^{\mu}=0$, we conclude $s \in \widetilde{\mathfrak{p}}$. (Note that $s \notin \mathfrak{p}$, hence the extension to $\widetilde{\mathfrak{p}}$ in the first part of the proof). So we can choose $r=s$ above in Eqn. (14), then by continuity, positivity and by ranging over all $m$, we conclude that

$$
\mathbf{u}(p)-\mathbf{e}(p)(\mathbf{e}(p) \cdot \mathbf{u}(p))=0 \quad \forall p \in C_{+}
$$

and as the second term is just the projection of $\mathbf{u}(p)$ in the direction of $\mathbf{p}$, this means $\mathbf{u}(p)$ must be proportional to $\mathbf{p}$ for all $p \in C_{+} \backslash 0$, i.e. $\mathbf{u}(p)=\mathbf{p} q(p)$, for some suitable scalar function $q$. Since $u_{0}(p)=\mathbf{e}(p) \cdot \mathbf{u}(p)=\|\mathbf{p}\| q(p)=p_{0} q(p), p \in C_{+}$, this means $u_{\mu}(p)=p_{\mu} q(p), p \in C_{+}$. By setting $r=0$, we obtain a similar result for $v$, and hence $f_{\mu}(p)=p_{\mu} h(p), p \in C_{+}$. The only restriction on $h$ is that $f \in \mathfrak{Z}_{(0)}$, since $f$ is automatically in $\mathfrak{p}$ by its form. Thus by the first part of the proof, $\mathfrak{p}_{0}$ consists of these functions, together with complex multiples of gradients, and this establishes the theorem.

5.13 Remark (i) In the proof above, the fact that $f \in \mathfrak{X}$ means that $h$ must be smooth away from the origin. Since $h$ is undefined at the origin in the proof, consider the behaviour of $f \in \mathfrak{p}_{0}$ at zero. Let $a \in C_{+} \backslash 0$, then by continuity of $f$ :

$$
\mathbf{f}(0)=\lim _{t \rightarrow 0^{+}} \mathbf{f}(t a)=\mathbf{a} \lim _{t \rightarrow 0^{+}} t h(t a)=\mathbf{a} \lim _{t \rightarrow 0^{+}} \frac{\mathbf{a} \cdot \mathbf{f}(t a)}{\|\mathbf{a}\|^{2}}=\mathbf{e}(a)(\mathbf{e}(a) \cdot \mathbf{f}(0))
$$


which can only be true for all $a$ if $f(0)=0$.

(ii) Now recall Maxwell's equations $F_{\mu \nu}, \nu(x)=0$. In the heuristic version of Gupta-Bleuler QEM, these need to be imposed as state conditions to define the physical field. Using the smearing formula for $A(f), F_{\mu \nu}, \nu(x)$ corresponds to the space

$$
\mathfrak{f}:=\left\{f \in \mathfrak{X} \mid f_{\mu}(p)=p_{\mu} p^{\nu} k_{\nu}(p), \quad p \in C_{+}, k \in \mathfrak{X}\right\} .
$$

By Proposition 5.12 we observe that $\mathfrak{f} \subset \mathfrak{p}_{0}$, and thus enforcing the second stage of constraints $\widetilde{\mathcal{U}}=\delta_{\mathfrak{p}_{0}}$ will also impose the Maxwell equations. Note however that the inclusion $\mathfrak{f} \subset \mathfrak{p}_{0}$ is proper, which we see as follows. Consider a line $t \rightarrow t a, a \in C_{+} \backslash 0$ and let $f \in \mathfrak{f}$ and $g \in \mathfrak{p}_{0}$, then $\lim _{t \rightarrow 0^{+}} \frac{d}{d t} f_{\mu}(t a)=0$, but $\lim _{t \rightarrow 0^{+}} \frac{d}{d t} g_{\mu}(t a)=a_{\mu} \lim _{t \rightarrow 0^{+}}\left(h(t a)+t \frac{d}{d t} h(t a)\right)$ if $g_{\mu}(p)=p_{\mu} h(p)$, and we can easily choose an $h \in \mathcal{S}\left(\mathbb{R}^{4}, \mathbb{C}\right)$ which makes the latter nonzero. Thus merely imposing the Maxwell equations does not appear to be sufficient to make the physical algebra simple (contrary to a claim in [8]).

(iii) In the next step below, we will factor out $\mathfrak{p}_{0}$ from $\mathfrak{p}$. Since $\mathfrak{p}_{0} \supset \mathbb{C} \cdot \mathfrak{G} \cap \mathfrak{Z}_{(0)}$, at this point we factor out the noncausal fields, and regain the reality condition of $\mathfrak{X}$.

(iv) From the characterisations of the spaces $\mathfrak{p}_{0}$ and $\mathfrak{p}$ above, we notice that the triple of spaces $\mathfrak{p}_{0} \subset \mathfrak{p} \subset \mathfrak{Y}$ corresponds with the one particle spaces of the triple of spaces in the heuristic theory $\mathcal{H}^{\prime \prime} \subset \mathcal{H}^{\prime} \subset \mathcal{H}$ hence a Fock-Krein construction on $\mathfrak{Y}$ (equipped with the right indefinite inner product) will reproduce the heuristic spaces. This is done explicitly in Subsection 5.6

For completeness we would also like to consider the local structure of the constraint system $\left(\mathcal{R}_{0}, \widetilde{\mathcal{U}}\right)$. Define $\widetilde{\mathcal{U}}(\Theta):=\widetilde{\mathcal{U}} \cap \mathcal{R}(\Theta)=\delta_{\mathfrak{s}(\Theta)}, \Theta \in \Gamma$, where $\mathfrak{s}(\Theta):=\mathfrak{p}_{0} \cap \mathfrak{p}(\Theta)$, then it is clear that $\Theta \rightarrow(\mathcal{R}(\Theta), \tilde{\mathcal{U}}(\Theta))$ is a system of local quantum constraints. Since $\widetilde{\mathcal{U}} \subset Z\left(\mathcal{R}_{0}\right)$, a local T-procedure produces:

$$
\begin{aligned}
\widetilde{\mathcal{D}}(\Theta) & =[\mathcal{R}(\Theta)(\mathbb{1}-\widetilde{\mathcal{U}}(\Theta))] \\
\widetilde{\mathcal{O}}(\Theta) & =\mathcal{R}(\Theta) \\
\widetilde{\mathcal{R}}(\Theta) & =\mathcal{R}(\Theta) /[\mathcal{R}(\Theta)(11-\tilde{\mathcal{U}}(\Theta))] .
\end{aligned}
$$

The main result of this section is:

5.14 Theorem The system of local constraints $\Theta \rightarrow(\mathcal{R}(\Theta), \widetilde{\mathcal{U}}(\Theta))$ satisfies reduction isotony, causality and weak covariance, hence $\Theta \rightarrow \widetilde{\mathcal{R}}(\Theta)$ is a HK-QFT. Moreover

$$
\widetilde{\mathcal{R}}(\Theta) \cong \overline{\Delta(\mathfrak{p}(\Theta) / \mathfrak{s}(\Theta), \widetilde{B})} \cong \overline{\Delta(\mathfrak{c}(\Theta), B)}
$$

where $\mathfrak{c}(\Theta):=\left\{f \in \mathfrak{p}(\Theta) \cap \mathfrak{X} \mid \mathbf{p} \cdot \mathbf{f}(p)=0, \quad p \in C_{+}\right\}$is the "Coulomb space", and

$$
\widetilde{\mathcal{R}}_{0} \cong \overline{\Delta\left(\mathfrak{p} / \mathfrak{p}_{0}, \widetilde{B}\right)} \cong \overline{\Delta(\mathfrak{c}, B)} \subset \overline{\Delta(\mathfrak{X}, B)}
$$

where $\widetilde{B}$ is $B$ factored to $\mathfrak{p} / \mathfrak{p}_{0}$, and $\mathfrak{c}:=\left\{f \in \mathfrak{p} \cap \mathfrak{X} \mid \mathbf{p} \cdot \mathbf{f}(p)=0, p \in C_{+}\right\}$.

Proof: For reduction isotony, since it is obvious that if $\Theta_{1} \subseteq \Theta_{2}$ then $\widetilde{\mathcal{O}}\left(\Theta_{1}\right)=\mathcal{R}\left(\Theta_{1}\right) \subseteq \mathcal{R}\left(\Theta_{2}\right)=$ $\widetilde{\mathcal{O}}\left(\Theta_{2}\right)$, we only need to show that $\widetilde{\mathcal{D}}\left(\Theta_{1}\right)=\widetilde{\mathcal{D}}\left(\Theta_{2}\right) \cap \mathcal{R}\left(\Theta_{1}\right)$, which by Lemma 3.8 will be the case if every Dirac state on $\mathcal{R}\left(\Theta_{1}\right)$ extends to a Dirac state on $\mathcal{R}\left(\Theta_{2}\right)$. We first prove that $\mathfrak{p}(\Theta)=$ $\mathfrak{c}(\Theta) \oplus \mathfrak{s}(\Theta)$ where $\mathfrak{c}(\Theta)$ is the "Coulomb space" above, and $\mathfrak{s}(\Theta):=\mathfrak{p}_{0} \cap \mathfrak{p}(\Theta)$. Let $m \in \mathfrak{p}(\Theta)$, so $0=p_{\mu} m^{\mu}(p), p \in C_{+}$and $m=f+n$ where $f \in \mathfrak{X}(\Theta) \cap \mathfrak{p}(\Theta)$ and $n \in \mathbb{C} \cdot \mathfrak{G} \cap \mathfrak{Z}(\Theta) \subset \mathfrak{s}(\Theta)$. 
Now write $f_{\mu}=g_{\mu}+p_{\mu} h$ where $h(p):=f_{0}(p) /\|\mathbf{p}\|=\mathbf{p} \cdot \mathbf{f} /\|\mathbf{p}\|^{2}$ and $g_{\mu}(p):=f_{\mu}(p)-p_{\mu} h(p)$. Then obviously $p_{\mu} h$ is in $\mathfrak{s}(\Theta)$ and $\mathbf{p} \cdot \mathbf{g}(p)=\mathbf{p} \cdot \mathbf{f}(p)-\|\mathbf{p}\|^{2} h(p)=0$, so $g \in \mathfrak{c}(\Theta)$. Thus we have a decomposition $m_{\mu}=g_{\mu}+\left(n_{\mu}+p_{\mu} h\right)$ where $g \in \mathfrak{c}(\Theta)$ and the function in the bracket is in $\mathfrak{s}(\Theta)$. To see that the decomposition is unique, let $g, k \in \mathfrak{c}(\Theta)$ such that $g_{\mu}-k_{\mu}=p_{\mu} h$. Then $0=\mathbf{p} \cdot(\mathbf{g}-\mathbf{k})=\|\mathbf{p}\|^{2} h$, i.e. $h=0$.

Since for $\Theta_{1} \subseteq \Theta_{2}$ we have $\mathfrak{s}\left(\Theta_{1}\right)=\mathfrak{s}\left(\Theta_{2}\right) \cap \mathfrak{p}\left(\Theta_{1}\right)$ and thus $\operatorname{Span}\left(\mathfrak{p}\left(\Theta_{1}\right) \cup \mathfrak{s}\left(\Theta_{2}\right)\right)=$ $\mathfrak{c}\left(\Theta_{1}\right) \oplus \mathfrak{s}\left(\Theta_{2}\right)$, so $\mathcal{A}:=\mathrm{C}^{*}\left(\delta_{\operatorname{Span}\left(\mathfrak{p}\left(\Theta_{1}\right) \cup \mathfrak{s}\left(\Theta_{2}\right)\right)}\right)$ is generated by two mutually commuting $\mathrm{C}^{*}$ algebras $\mathrm{C}^{*}\left(\delta_{\mathfrak{c}\left(\Theta_{1}\right)}\right)$ and $\mathrm{C}^{*}\left(\delta_{\mathfrak{s}\left(\Theta_{2}\right)}\right)$ where the last one is commutative. Now let $A \in \mathrm{C}^{*}\left(\delta_{\mathfrak{c}\left(\Theta_{1}\right)}\right)$ and $B \in \mathrm{C}^{*}\left(\delta_{\mathfrak{s}\left(\Theta_{2}\right)}\right)$ such that $A B=0$. Then we want to show that $A=0$ or $B=0$. Let

$$
\begin{array}{rlrl}
A_{n} & :=\sum_{i=1}^{N_{n}} \alpha_{i}^{(n)} \delta_{f_{i}} \longrightarrow A=\sum_{i=1}^{\infty} \alpha_{i} \delta_{f_{i}} & \text { where } & f_{i} \in \mathfrak{c}\left(\Theta_{1}\right), \quad \text { and } \\
B_{n}:=\sum_{j=1}^{M_{n}} \beta_{j}^{(n)} \delta_{k_{j}} \longrightarrow B=\sum_{j=1}^{\infty} \beta_{j} \delta_{k_{j}} & \text { with } & k_{j} \in \mathfrak{s}\left(\Theta_{2}\right)
\end{array}
$$

Then $0=A B=\lim _{n \rightarrow \infty} \sum_{i, j}^{N_{n}, M_{n}} \alpha_{i}^{(n)} \beta_{j}^{(n)} \delta_{f_{i}+k_{j}}$. However $f_{i}+k_{j} \neq f_{i^{\prime}}+k_{j^{\prime}}$ for $i \neq i^{\prime}$ and $j \neq j^{\prime}$ since $\mathfrak{c}\left(\Theta_{1}\right)$ and $\mathfrak{s}\left(\Theta_{2}\right)$ are linear independent spaces intersecting only in $\{0\}$. Thus the set $\left\{\delta_{f_{i}+k_{j}} \mid i \in \mathbb{N}, j \in \mathbb{N}\right\}$ is linearly independent and so $0=\lim _{n \rightarrow \infty} \alpha_{i}^{(n)} \beta_{j}^{(n)}=\alpha_{i} \beta_{j}$. Since this holds for all possible pairs $i, j$, there is no pair $\alpha_{i}, \beta_{j}$ such that $\alpha_{i} \beta_{j} \neq 0$ and so either all $\alpha_{i}=0$ or all $\beta_{j}=0$, i.e. $A=0$ or $B=0$. Thus from Takesaki [28, Exercise 2, p. 220] we conclude that $\mathcal{A}$ is isomorphic to $\mathrm{C}^{*}\left(\delta_{\mathfrak{c}\left(\Theta_{1}\right)}\right) \otimes \mathrm{C}^{*}\left(\delta_{\mathfrak{s}\left(\Theta_{2}\right)}\right)$ by the map $\varphi(A \otimes B):=A B$.

Let $\omega$ be a Dirac state on $\mathcal{R}\left(\Theta_{1}\right)$, i.e. $\omega\left(\delta_{\mathfrak{s}\left(\Theta_{1}\right)}\right)=1$, and then define a state $\widetilde{\omega}$ on $\mathcal{A}$ by $\widetilde{\omega}:=(\omega \otimes \widehat{\omega}) \circ \varphi^{-1}$ where $\widehat{\omega}$ is the state on $C^{*}\left(\delta_{\mathfrak{s}\left(\Theta_{2}\right)}\right)$ satisfying $\widehat{\omega}\left(\delta_{\mathfrak{s}\left(\Theta_{2}\right)}\right)=1$. Now extend $\widetilde{\omega}$ arbitrarily to $\mathcal{R}\left(\Theta_{2}\right) \supset \mathcal{A}$, then it coincides with $\omega$ on $\mathcal{R}\left(\Theta_{1}\right)$ and satisfies $\widehat{\omega}\left(\delta_{\mathfrak{s}\left(\Theta_{2}\right)}\right)=1$ hence is a Dirac state on $\mathcal{R}\left(\Theta_{2}\right)$. This establishes reduction isotony.

For causality, the fact that $\Theta \rightarrow \mathcal{R}(\Theta)$ is a HK-QFT already implies that $\left[\mathcal{R}\left(\Theta_{1}\right), \mathcal{R}\left(\Theta_{2}\right)\right]=0$ when $\Theta_{1} \perp \Theta_{2}$, so $\left[\widetilde{\mathcal{O}}\left(\Theta_{1}\right), \widetilde{\mathcal{O}}\left(\Theta_{2}\right)\right]=0$.

For covariance, we already have that $\alpha_{g}(\widetilde{\mathcal{O}}(\Theta))=\alpha_{g}(\mathcal{R}(\Theta))=\mathcal{R}(g \Theta)=\widetilde{\mathcal{O}}(g \Theta)$ for $g \in \mathcal{P}_{+}^{\uparrow}$, $\Theta \in \Gamma$. Now

$$
\alpha_{g}(\tilde{\mathcal{U}}(\Theta))=\alpha_{g}\left(\delta_{\mathfrak{s}(\Theta)}\right)=\delta_{V_{g} \mathfrak{s}(\Theta)} \quad \text { and } \quad \tilde{\mathcal{U}}(g \Theta)=\delta_{\mathfrak{s}(g \Theta)} .
$$

To see that these are equal, note that $V_{g} \mathfrak{s}(\Theta)=V_{g}\left(\mathfrak{p}_{0} \cap \mathfrak{p}(\Theta)\right), V_{g}$ is symplectic, and $V_{g} \mathfrak{p}(\Theta) \subseteq$ $\mathfrak{p}(g \Theta)$ by

$$
p^{\mu}\left(V_{g} f\right)_{\mu}(p)=p^{\mu}\left(\Lambda f\left(\Lambda^{-1} p\right)\right)_{\mu} e^{-i p \cdot a}=\left(\Lambda^{-1} p\right)^{\mu} f_{\mu}\left(\Lambda^{-1} p\right) e^{-i p \cdot a}=0
$$

for $p \in C_{+}$and $f \in \mathfrak{p}(\Theta)$. Thus $V_{g}\left(\mathfrak{p}_{0} \cap \mathfrak{p}(\Theta)\right) \subseteq \mathfrak{p}_{0} \cap \mathfrak{p}(g \Theta)$. For the reverse inclusion: $V_{g^{-1}}\left(\mathfrak{p}_{0} \cap \mathfrak{p}(g \Theta)\right) \subseteq \mathfrak{p}_{0} \cap \mathfrak{p}(\Theta)$ implies that $\mathfrak{p}_{0} \cap \mathfrak{p}(g \Theta) \subseteq V_{g}\left(\mathfrak{p}_{0} \cap \mathfrak{p}(\Theta)\right)$. Thus $\alpha_{g}(\widetilde{\mathcal{U}}(\Theta))=\widetilde{\mathcal{U}}(g \Theta)$.

Finally, for the last two isomorphism claims, recall that $\mathcal{R}(\Theta)=\mathrm{C}^{*}\left(\delta_{\mathfrak{p}(\Theta)}\right)=\overline{\Delta(\mathfrak{p}(\Theta), B)}$ and so since $\widetilde{\mathcal{R}}(\Theta)=\mathcal{R}(\Theta) /[\mathcal{R}(\Theta)(\mathbb{1}-\widetilde{\mathcal{U}}(\Theta))]$ and $\widetilde{\mathcal{U}}(\Theta)=\delta_{\mathfrak{s}(\Theta)}$ where $\mathfrak{s}(\Theta)$ is the degenerate part of $\mathfrak{p}(\Theta)$, we conclude from Theorem 4.5 that $\widetilde{\mathcal{R}}(\Theta) \cong \overline{\Delta(\mathfrak{p}(\Theta) / \mathfrak{s}(\Theta), \widetilde{B})}$ (providing the symplectic commutant of $\mathfrak{s}(\Theta)$ in $\mathfrak{p}(\Theta), \mathfrak{s}(\Theta)^{\prime}=\mathfrak{p}(\Theta)$, and this is obvious since $\mathfrak{s}(\Theta)=\mathfrak{p}_{0} \cap \mathfrak{p}(\Theta)$ and $\mathfrak{p}(\Theta) \subset \mathfrak{p})$. Since $\mathfrak{p}(\Theta)=\mathfrak{c}(\Theta) \oplus \mathfrak{s}(\Theta)$, this is isomorphic to $\overline{\Delta(\mathfrak{c}(\Theta), B)}$. Since for $\Theta_{1} \subseteq \Theta_{2}$ the inclusion $\widetilde{\mathcal{R}}\left(\Theta_{1}\right) \subseteq \widetilde{\mathcal{R}}\left(\Theta_{2}\right)$ comes from $\mathfrak{p}\left(\Theta_{1}\right) \subseteq \mathfrak{p}\left(\Theta_{2}\right)$, and this inclusion factors through to produce $\mathfrak{p}\left(\Theta_{1}\right) \subseteq \mathfrak{p}\left(\Theta_{2}\right)$, hence $\mathfrak{c}\left(\Theta_{1}\right) \subseteq \mathfrak{c}\left(\Theta_{2}\right)$, so the last isomorphism is clear.

Thus the quasi-local algebra $\widetilde{\mathcal{R}}_{0}$ is simple. Below we will show that for double cones $\Theta$ the local algebras are also simple. For a more general net $\Gamma$ it is not clear whether the local algebras are simple. 
5.15 Remark An apparent puzzle raised by the isomorphisms $\widetilde{\mathcal{R}}(\Theta) \cong \overline{\Delta(\mathfrak{c}(\Theta), B)}$ here, is the noncovariance of the spaces $\mathfrak{c}(\Theta)$ under $V_{g}, g \in \mathcal{P}_{+}^{\uparrow}$, given that the net $\widetilde{\mathcal{R}}(\Theta)$ is covariant under the isomorphisms derived from $V_{g}$. The resolution is that $V_{g}$ maps an equivalence class $f+\mathfrak{s}(\Theta)$ in $\mathfrak{p}(\Theta)$ to the equivalence class $V_{g} f+\mathfrak{s}(g \Theta)$ in $\mathfrak{p}(g \Theta)$, and these equivalence classes correspond to elements $h \in \mathfrak{c}(\Theta)$ and $k \in \mathfrak{c}(g \Theta)$ repectively, but it is not true that $k=V_{g} h$.

5.16 Theorem If the sets $\Theta \in \Gamma$ consist of double cones, then the local algebras

$$
\widetilde{\mathcal{R}}_{0}(\Theta) \cong \overline{\Delta(\mathfrak{p}(\Theta) / \mathfrak{s}(\Theta), \widetilde{B})} \cong \overline{\Delta(\mathfrak{c}(\Theta), B)}
$$

are simple.

Proof: By Theorems 4.2 and 5.14 it suffices to prove that $(\mathfrak{c}(\Theta), B)$ is a nondegenerate symplectic space for each double cone $\Theta$, where we consider

$$
\begin{aligned}
\mathfrak{c}(\Theta) & :=\left\{f \in \mathfrak{p}(\Theta) \cap \mathfrak{X} \mid \mathbf{p} \cdot \mathbf{f}(p)=0, \quad p \in C_{+}\right\} \\
\mathfrak{C}(\Theta) & :=\left\{f \in C_{c}^{\infty}\left(\mathbb{R}^{4}, \mathbb{R}^{4}\right) \mid f_{0}=0, \sum \partial_{\ell} f_{\ell}=0, \quad \text { and } \quad \operatorname{supp} f \subset \Theta\right\} \\
\mathfrak{C} & :=\left\{f \in C_{c}^{\infty}\left(\mathbb{R}^{4}, \mathbb{R}^{4}\right) \mid f_{0}=0 \text { and } \sum \partial_{\ell} f_{\ell}=0\right\} .
\end{aligned}
$$

Observe that if we define the map $\rho: \mathcal{S}\left(\mathbb{R}^{4}, \mathbb{R}^{4}\right) \rightarrow \mathfrak{X}=\mathcal{S}\left(\mathbb{R}^{4}, \mathbb{R}^{4}\right) / \operatorname{Ker}(D)$ by $\rho(f)=\widehat{f}+\operatorname{Ker}(D)$, then $\mathfrak{c}(\Theta)=\rho(\mathfrak{C}(\Theta))$.

We adapt the arguments in Dimock [30]. (Note though that from Proposition 5.12 we do not need the assumption that the Cauchy surface is compact, used in [30, Proposition 5]). For test functions in $\mathcal{S}\left(\mathbb{R}^{4}, \mathbb{R}^{4}\right)$ we have

$$
\begin{aligned}
\widehat{D}(f, h) & :=D(\widehat{f}, \widehat{h})=\iint f_{\mu}(x) h^{\mu}(y) D(x-y) d^{4} x d^{4} y \\
& =\int f_{\mu}(x)(D h)^{\mu}(x) d^{4}(x) \\
\text { where } \quad(D h)_{\mu}(x) & :=\int h_{\mu}(y) D(x-y) d^{4} y \\
& =-i \pi \int_{C_{+}} \frac{d^{3} p}{p_{0}}\left(e^{i p \cdot x} \widehat{h}_{\mu}(p)-e^{-i p \cdot x} \widehat{\widehat{h}}_{\mu}(p)\right) .
\end{aligned}
$$

Note that $D$ is the difference of the retarded and advanced fundamental solutions of the wave operator $\square$, hence $D f$ is a solution of the wave equation (cf. 32, 31]). Henceforth we will only consider test functions in $\mathfrak{C}$. We want to express $D(\widehat{f}, \widehat{h})$ in terms of the corresponding real Cauchy data. Given $f \in \mathfrak{C}$, we define these by:

$$
\begin{aligned}
Q_{\ell}^{f}(\mathbf{x}) & :=\frac{-1}{\pi}(D f)_{\ell}(0, \mathbf{x}) \in C_{c}^{\infty}\left(\mathbb{R}^{3}, \mathbb{R}\right) \\
R_{\ell}^{f}(\mathbf{x}) & :=\frac{1}{\pi}\left(\partial_{0}(D f)_{\ell}\right)(0, \mathbf{x}) \in C_{c}^{\infty}\left(\mathbb{R}^{3}, \mathbb{R}\right), \quad \ell=1,2,3 .
\end{aligned}
$$

Then their Fourier transforms are, using the conventions $\widehat{f}(p)=\int_{\mathbb{R}^{4}} e^{-i p \cdot x} f(x) d^{4} x$ and $\widehat{h}(\mathbf{p})=$ $(2 \pi)^{-3 / 2} \int_{\mathbb{R}^{3}} e^{i \mathbf{p} \cdot \mathbf{x}} h(\mathbf{x}) d^{3} x$ for four and three dimensional Fourier transforms:

$$
\begin{aligned}
& \widehat{Q_{\ell}^{f}}(\mathbf{p})=\frac{i(2 \pi)^{3 / 2}}{\|\mathbf{p}\|}\left(\widehat{f}_{\ell}(\|\mathbf{p}\|, \mathbf{p})-\overline{\widehat{f}}_{\ell}(\|\mathbf{p}\|,-\mathbf{p})\right) \\
& \widehat{R_{\ell}^{f}}(\mathbf{p})=(2 \pi)^{3 / 2}\left(\widehat{f}_{\ell}(\|\mathbf{p}\|, \mathbf{p})+\overline{\widehat{f}}_{\ell}(\|\mathbf{p}\|,-\mathbf{p})\right) .
\end{aligned}
$$


If we substitute these into the rhs of the equation

$$
\int_{\mathbb{R}^{3}}\left(Q_{\ell}^{f} R_{\ell}^{h}-R_{\ell}^{f} Q_{\ell}^{h}\right)(\mathbf{x}) d^{3} x=\int_{\mathbb{R}^{3}}\left(\widehat{Q_{\ell}^{f}}(-\mathbf{p}) \widehat{R_{\ell}^{h}}(\mathbf{p})-\widehat{R_{\ell}^{f}}(-\mathbf{p}) \widehat{Q_{\ell}^{h}}(\mathbf{p})\right) d^{3} p
$$

(summation over $\ell$ ), then we find with some algebraic work that

$$
\widehat{D}(f, h)=\frac{-1}{16 \pi^{2}} \int_{\mathbb{R}^{3}}\left(Q_{\ell}^{f} R_{\ell}^{h}-R_{\ell}^{f} Q_{\ell}^{h}\right)(\mathbf{x}) d^{3} x .
$$

Now let $\Theta$ be a double cone, by covariance we can assume it to be centered at the origin. Let $\Sigma$ be its intersection with the Cauchy surface $t=0$. Then if $f \in \mathfrak{C}(\Theta)$ we have by the properties of $D$ that $\operatorname{supp} Q_{\ell}^{f} \subset \Sigma \supset \operatorname{supp} R_{\ell}^{f}$ (cf. [32, 31]). Further from the arguments in the proof of Proposition 2 in [30] we know that for any pair $(Q, R) \in C_{c}^{\infty}\left(\mathbb{R}^{3}, \mathbb{R}^{3}\right) \times C_{c}^{\infty}\left(\mathbb{R}^{3}, \mathbb{R}^{3}\right)$ satisfying $\partial_{\ell} Q_{\ell}=0=\partial_{\ell} R_{\ell}$, there exists a unique solution of the wave equation $\varphi \in C^{\infty}\left(\mathbb{R}^{4}, \mathbb{R}^{4}\right)$ with these data and satisfying $\partial_{\ell} \varphi_{\ell}=0=\varphi_{0}$. Even more by [30, Proposition 4(c)] we can always find an $f \in \mathfrak{C}(\Theta)$ such that $D f=\varphi$.

Now take a test function $h \in \mathfrak{C}(\Theta)$ such that $\rho(h) \in \operatorname{Ker}(B \uparrow \mathfrak{c}(\Theta))$, i.e.

$$
0=\int_{\Sigma}\left(Q_{\ell}^{f} R_{\ell}^{h}-R_{\ell}^{f} Q_{\ell}^{h}\right)(\mathbf{x}) d^{3} x=\int_{\mathbb{R}^{3}}\left(Q_{\ell}^{f} R_{\ell}^{h}-R_{\ell}^{f} Q_{\ell}^{h}\right)(\mathbf{x}) d^{3} x
$$

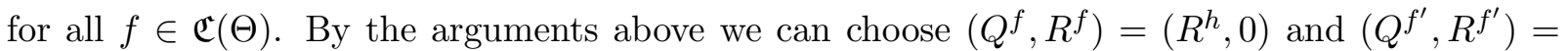
$\left(0,-Q^{h}\right)$ to conclude that $\left(Q^{h}, R^{h}\right)=(0,0)$. Then by uniqueness this implies that $D h=0$, i.e. $\rho(h)=0$.

In this example we have done our constraint reduction in two stages, and the question arises as to whether we would have obtained the same physical algebra from a single reduction by the full set of constraints. This will be examined in the next main section.

\subsection{Connecting with the indefinite inner product}

In this subsection we want to connect the $\mathrm{C}^{*}$-algebraic version above of Gupta-Bleuler electromagnetism with the usual one on indefinite inner product space (henceforth abbreviated to IIPspace), sketched in Subsect. 5.1. We will freely use the Fock-Krein construction of Mintchev [34]. Start with the space

$$
\begin{aligned}
\mathfrak{Y} & =\mathcal{S}\left(\mathbb{R}^{4}, \mathbb{C}^{4}\right) / \operatorname{Ker}(D) \\
\text { with IIP: } \quad K(f, h) & :=-2 \pi \int_{C_{+}} \overline{f^{\mu}(p)} h_{\mu}(p) \frac{d^{3} p}{p_{0}}, \quad \forall f, h \in \mathfrak{Y},
\end{aligned}
$$

which is well-defined on $\mathfrak{Y}$ because $\operatorname{Ker}(D)=\operatorname{Ker}(K)$. Note that $B=\operatorname{Im} K$. Define now on $\mathfrak{Y}$ the operator $J$ by $(J f)_{0}=f_{0},(J f)_{\ell}=-f_{\ell}, \ell=1,2,3$, then obviously $J^{2}=\mathbb{1}$ and $(f, h):=K(f, J h)$ defines a positive definite inner product on $\mathfrak{Y}$. Let $\mathcal{N}$ be the Hilbert space completion w.r.t. this inner product (so in fact, it is just $L^{2}\left(C_{+}, \mathbb{C}^{4}, \mu_{0}\right)$ with $d \mu_{0}=d^{3} p / p_{0}$ ) and let $\mathfrak{F}(\mathcal{N})$ be the symmetric Fock space constructed on $\mathcal{N}$. Below we will use the notation $\mathfrak{F}_{0}(\mathcal{L})$ for the finite particle space with entries taken from a given space $\mathcal{L} \subset \mathcal{N}$, and as usual $\mathfrak{F}_{0}:=\mathfrak{F}_{0}(\mathcal{N})$. We make $\mathfrak{F}(\mathcal{N})$ into a Krein space with the IIP $\langle\psi, \varphi\rangle:=(\psi, \Gamma(J) \varphi)$ where $\Gamma(J)$ is the second quantization of $J$ and the round brackets indicate the usual Hilbert space inner product. We define creation and annihilation operators as usual, except for the replacement of the inner product by the IIP, i.e. on the n-particle space $\mathcal{H}^{(n)}$ they are

$$
\begin{aligned}
a^{\dagger}(f) S_{n} h_{1} \otimes \cdots \otimes h_{n} & =\sqrt{n+1} S_{n+1} f \otimes h_{1} \otimes \cdots \otimes h_{n} \\
a(f) S_{n} h_{1} \otimes \cdots \otimes h_{n} & =\frac{1}{\sqrt{n}} \sum_{i=1}^{n}\left\langle f, h_{i}\right\rangle S_{n-1} h_{1} \otimes \cdots \tilde{h}_{i} \cdots \otimes h_{n}
\end{aligned}
$$


where the tilde means omission and $S_{n}$ is the symmetrisation operator for $\mathcal{H}^{(n)}$. Note that $a^{\dagger}(f)$ is the $\langle\cdot, \cdot\rangle$-adjoint of $a(f)$, not the Hilbert space adjoint. The connection with the heuristic creation and annihilation operators in Subsect. 5.1 comes from the smearing formula

$$
a(f)=\sqrt{2 \pi} \int_{C_{+}} a_{\mu}(\mathbf{p}) f^{\mu}(p) \frac{d^{3} p}{p_{0}}
$$

and the Krein adjoint formula for $a^{\dagger}(f)$ (which produces a complex conjugation on the smearing function). Then the constructed operators have the correct commutation relations, so that if we define the field operator by

$$
\begin{aligned}
A(f) & :=\frac{1}{\sqrt{2}}\left(a^{\dagger}(f)+a(f)\right) \\
\text { then: } \quad[A(f), A(h)] & =i B(f, h) .
\end{aligned}
$$

We only need to restrict to $f \in \mathfrak{X}$ to make the connection with the field operators of before. (Note that since $\mathfrak{Y}$ is the complex span of $\mathfrak{X}$, the complex span of the set $\{A(f) \Omega \mid f \in \mathfrak{X}\}$ is dense in $\mathfrak{F}(\mathcal{N})$.) Following Mintchev [34] we now define on the finite particle space $\mathfrak{F}_{0}$ the unbounded $\langle\cdot, \cdot\rangle$-unitary operators

$$
W(f) \psi:=\lim _{N \rightarrow \infty} \sum_{k=0}^{N} \frac{[i A(f)]^{k}}{k !} \psi
$$

which satisfy the Weyl relations, and hence constitute a Krein representation $\gamma$ by $\gamma\left(\delta_{f}\right):=W(f)$, of the dense ${ }^{*}$-algebra generated by $\delta_{\mathfrak{Y}}$ in $\overline{\Delta(\mathfrak{Y}, B)}$, usually denoted by $\Delta(\mathfrak{Y}, B)$. Note that $\gamma: \Delta(\mathfrak{Y}, B) \rightarrow \mathrm{Op}\left(\mathfrak{F}_{0}\right)$ takes the $\mathrm{C}^{*}$-involution to the Krein involution. Moreover, for the constraints we see from the heuristic formula: $\chi(h):=a\left(i p_{\mu} \widehat{h} \sqrt{\pi}\right)$, so the set $\left\{\chi(h) \mid h \in \mathcal{S}\left(\mathbb{R}^{4}, \mathbb{R}\right)\right\}$ corresponds to $\{a(f) \mid f \in \mathfrak{G}\}$. By the commutation relations, we still have

$$
\left[\chi(h)^{\dagger} \chi(h), A(f)\right]=i A\left(G_{h}(f)\right) .
$$

We want to extend $\gamma$ so that it also represents the constraint unitaries $U_{\mathcal{G}}$.

5.17 Proposition $\quad T_{h}^{t}: \mathfrak{Y} \rightarrow \mathfrak{Y}$ is K-unitary, i.e. $K(f, g)=K\left(T_{h}^{t} f, T_{h}^{t} g\right)$ for all $f, g, h, t$.

Proof:

$$
\begin{aligned}
& K\left(T_{h}^{t} f, T_{h}^{t} g\right)=-2 \pi \int_{C_{+}} \frac{d^{3} p}{p_{0}}\left\{\left(\overline{f_{\mu}}+i t \pi p_{\mu} \overline{\widehat{h}} \overline{c(f, h)}\right) \cdot\left(g^{\mu}-i t \pi p^{\mu} \widehat{h} c(g, h)\right)\right\} \\
& =K(f, g)-2 \pi^{2} i t \int_{C_{+}} \frac{d^{3} p}{p_{0}} p^{\mu}\left(g_{\mu} \overline{\widehat{h}} \overline{c(f, h)}-\overline{f_{\mu}} \widehat{h} c(g, h)\right)
\end{aligned}
$$

and the last integral is:

$$
\begin{aligned}
& \left(\int_{C_{+}} \frac{d^{3} p}{p_{0}} p_{\mu} g^{\mu} \overline{\widehat{h}}(p)\right)\left(\int_{C_{+}} \frac{d^{3} p^{\prime}}{p_{0}^{\prime}} p_{\nu}^{\prime} \overline{f^{\nu}} \widehat{h}\left(p^{\prime}\right)\right) \\
& \quad-\left(\int_{C_{+}} \frac{d^{3} p}{p_{0}} p_{\mu} \overline{f^{\mu}} \widehat{h}(p)\right)\left(\int_{C_{+}} \frac{d^{3} p^{\prime}}{p_{0}^{\prime}} p_{\nu}^{\prime} g^{\nu} \overline{\widehat{h}}\left(p^{\prime}\right)\right) \\
& =0 .
\end{aligned}
$$

Thus we know from Mintchev [34] the second quantized operator $\Gamma\left(T_{h}^{t}\right)$ is well-defined on $\mathfrak{F}_{0}(\mathfrak{Y})$, it is $\langle\cdot, \cdot\rangle$-unitary, and it implements $T_{h}^{t}$ on $A(f)$. By the definition of $\Gamma\left(T_{h}^{t}\right)$ it is also clear that the set of these commute, and thus we can extend $\gamma$ to $U_{\mathcal{G}}$ by defining $\gamma\left(U_{T_{h}^{t}}\right):=\Gamma\left(T_{h}^{t}\right)$. For the heuristic theory, we would like to identify this with $\exp \left(i t \chi(h)^{\dagger} \chi(h)\right)$, and this is done in the next proposition. 


\subsection{Proposition}

$$
\left.\frac{d}{d t} \Gamma\left(T_{h}^{t}\right) \psi\right|_{t=0}=i \chi(h)^{\dagger} \chi(h) \psi \quad \forall \psi \in \mathfrak{F}_{0}(\mathfrak{Y}), h \in \mathcal{S}\left(\mathbb{R}^{4}, \mathbb{R}\right) .
$$

Proof: Let $\psi=S_{n} f_{1} \otimes \cdots \otimes f_{n}$ with $f_{i} \in \mathfrak{Y}$, and recall that $T_{h}^{t} f=f+t G_{h}(f)$. Then

$$
\begin{gathered}
\left.\quad \frac{d}{d t} \Gamma\left(T_{h}^{t}\right) S_{n} f_{1} \otimes \cdots \otimes f_{n}\right|_{t=0}=\left.\frac{d}{d t} S_{n}\left(T_{h}^{t} f_{1}\right) \otimes \cdots \otimes\left(T_{h}^{t} f_{n}\right)\right|_{t=0} \\
=\quad S_{n} G_{h}\left(f_{1}\right) \otimes f_{2} \otimes \cdots \otimes f_{n}+\cdots+S_{n} f_{1} \otimes \cdots \otimes f_{n-1} \otimes G_{h}\left(f_{n}\right) .
\end{gathered}
$$

On the other hand, if we start from the right hand side of the claim in the proposition, and use

$$
G_{h}\left(f_{k}\right)=-i \pi p_{\mu} \widehat{h}(p) \int_{C_{+}} \frac{d^{3} p^{\prime}}{p_{0}^{\prime}} f_{k}^{\nu} p_{\nu}^{\prime} \overline{\widehat{h}}\left(p^{\prime}\right)=\pi p_{\mu} \widehat{h}(p)\left\langle i p_{\nu} \widehat{h}, f_{k}\right\rangle
$$

then we see that

$$
\begin{aligned}
i \chi(h)^{\dagger} \chi(h) S_{n} f_{1} \otimes \cdots \otimes f_{n}=i \pi a^{\dagger}\left(i p_{\mu} \widehat{h}\right) a\left(i p_{\mu} \widehat{h}\right) S_{n} f_{1} \otimes \cdots \otimes f_{n} \\
\quad=i \pi a^{\dagger}\left(i p_{\mu} \widehat{h}\right) \frac{1}{\sqrt{n}} \sum_{k=1}^{n}\left\langle i p_{\mu} \widehat{h}, f_{k}\right\rangle S_{n-1} f_{1} \otimes \cdots \widetilde{f_{k}} \cdots \otimes f_{n} \\
=i \pi \sum_{k=1}^{n}\left\langle i p_{\mu} \widehat{h}, f_{k}\right\rangle S_{n}\left(i p_{\mu} \widehat{h}\right) \otimes f_{1} \otimes \cdots \widetilde{f_{k}} \cdots \otimes f_{n} \\
=S_{n} G_{h}\left(f_{1}\right) \otimes f_{2} \otimes \cdots \otimes f_{n}+\cdots+S_{n} f_{1} \otimes \cdots \otimes G_{h}\left(f_{n}\right)
\end{aligned}
$$

and so comparing this with Eqn. (16) establishes the proposition.

Thus if $\mathcal{E}$ denotes the ${ }^{*}$-algebra generated by $\Delta(\mathfrak{Y}, B) \cup U_{\mathcal{G}}\left(\right.$ dense in $\left.\mathcal{F}_{e}\right)$, and we set $\gamma\left(U_{g}\right):=$ $\Gamma\left(U_{g}\right)$, then we now have a representation $\gamma: \mathcal{E} \rightarrow \mathrm{Op}\left(\mathfrak{F}_{0}(\mathfrak{Y})\right)$ which agrees with the GuptaBleuler operator theory.

To conclude this section we wish to compare the physical algebra obtained by $\mathrm{C}^{*}$-methods with the results of the spatial constraining in the usual theory. In the latter one defines

$$
\mathcal{H}^{\prime}:=\left\{\psi \in \mathfrak{F}(\mathcal{N}) \mid \psi \in \operatorname{Dom}(\chi(h)) \quad \text { and } \quad \chi(h) \psi=0 \quad \forall h \in \mathcal{S}\left(\mathbb{R}^{4}, \mathbb{R}\right)\right\}
$$

so if we take $\operatorname{Dom}(\chi(h))=\mathfrak{F}_{0}(\mathfrak{Y})$, then

\subsection{Proposition $\quad \mathcal{H}^{\prime}=\mathfrak{F}_{0}(\mathbb{C} \cdot \mathfrak{p})$.}

Proof: Since $\chi(h): \mathcal{H}^{(n)} \rightarrow \mathcal{H}^{(n-1)}$ and the $\mathrm{n}$-particle spaces are linearly independent, it suffices to check the condition $\chi(h) \psi=0$ on each $\mathcal{H}^{(n)}$ separately. Write

$$
\psi \in \mathcal{H}^{\prime} \cap \mathfrak{F}_{0}(\mathfrak{Y}) \quad \text { in the form: } \quad \psi=\sum_{k=1}^{N} S_{n} f_{k 1} \otimes \cdots \otimes f_{k n} .
$$

So $\psi \in \mathcal{H}^{\prime}$ means $\chi(h) \psi=0$, which implies $\left\langle\chi(h)^{\dagger} \varphi, \psi\right\rangle=0$ for all $h \in \mathcal{S}\left(\mathbb{R}^{4}, \mathbb{R}\right)$ and $\varphi \in \mathcal{H}^{(n-1)}$. Explicitly:

$$
\begin{aligned}
0 & =\left\langle a^{\dagger}\left(i p_{\mu} \widehat{h}\right) \varphi, \psi\right\rangle \\
& =\left\langle a^{\dagger}\left(i p_{\mu} \widehat{h}\right) \sum_{k=1}^{N} S_{n-1} g_{k 1} \otimes \cdots \otimes g_{k(n-1)}, \sum_{j=1}^{M} S_{n} f_{j 1} \otimes \cdots \otimes f_{j n}\right\rangle \\
& =\sum_{k=1}^{N} \sum_{j=1}^{M} \sqrt{n}\left\langle\left(i p_{\mu} \widehat{h}\right) \otimes g_{k 1} \otimes \cdots \otimes g_{k(n-1)}, S_{n} f_{j 1} \otimes \cdots \otimes f_{j n}\right\rangle \\
& =\sum_{k=1}^{N} \sum_{j=1}^{M} \sum_{\sigma \in P_{n}} \frac{\sqrt{n}}{n !}\left\langle i p_{\mu} \widehat{h}, f_{j \sigma(1)}\right\rangle\left\langle g_{k 1}, f_{j \sigma(2)}\right\rangle \cdots\left\langle g_{k(n-1)}, f_{j \sigma(n)}\right\rangle
\end{aligned}
$$


where $P_{n}$ denotes the permutation group on $\{1, \ldots, n\}$. This must hold for all $\varphi$ so if we let the $g_{k i}$ vary over $\mathfrak{Y}$, we get that $\left\langle i p_{\mu} \widehat{h}, f_{j i}\right\rangle=0$ for all $h$. Thus $0=\int_{C_{+}} \frac{d^{3} p}{p_{0}} p_{\mu} f_{j i}^{\mu} \widehat{\widehat{h}}(p)$ for all $h$, and the choice $\widehat{h}=i p_{\mu} f_{j i}^{\mu}$ then implies $p_{\mu} f_{j i}^{\mu} \mid C_{+}=0$ for all $f_{j i}$ i.e. $f_{j i} \in \mathfrak{p}$. Thus $\mathcal{H}^{\prime} \subseteq \mathfrak{F}_{0}(\mathbb{C} \cdot \mathfrak{p})$. The reverse inclusion is obvious.

Note that $\mathcal{H}^{\prime}=\mathfrak{F}_{0}(\mathbb{C} \cdot \mathfrak{p})$ is of course preserved by $A(\mathfrak{p} \cap \mathfrak{X})$, the generators of $W(\mathfrak{p} \cap \mathfrak{X})=\gamma\left(\delta_{\mathfrak{p} \cap \mathfrak{X}}\right)$. Thus by the exponential series, $W(\mathfrak{p} \cap \mathfrak{X})$ maps $\mathcal{H}^{\prime}$ into its Hilbert space closure. Since obviously $\Gamma\left(T_{h}^{t}\right) \mid \mathcal{H}^{\prime}=\mathbb{1}$, when we restrict the algebra $\gamma(\mathcal{E})$ to $\mathcal{H}^{\prime}$, the constraints are factored out. We already know that $\mathfrak{p} \cap \mathfrak{X}$ contains the smearing functions which produce the fields $F_{\mu \nu}$. We check that the IIP is positive semidefinite on $\mathcal{H}^{\prime}$. First, the one-particle space.

5.20 Proposition We have $K(f, f) \geq 0 \forall f \in \mathfrak{p} \quad$ and $\quad \operatorname{Ker}\left(K\lceil\mathfrak{p})=\mathfrak{p}_{0}\right.$.

Proof: Let $f \in \mathfrak{p}$, then by Theorem 5.5 we see $p_{\mu} f^{\mu}(p)=0$ for all $p \in C_{+}$, and thus by the proof of Proposition 5.12, $f_{0}(p)=\mathbf{p} \cdot \mathbf{f}(p) /\|\mathbf{p}\|$ for $p \neq 0$. Thus

$$
K(f, f):=-2 \pi \int_{C_{+}} \overline{f^{\mu}(p)} f_{\mu}(p) \frac{d^{3} p}{p_{0}}=2 \pi \int_{C_{+}}\left(\overline{\mathbf{f}} \cdot \mathbf{f}(p)-\left|f_{0}(p)\right|^{2}\right) \frac{d^{3} p}{p_{0}} .
$$

Now

$$
\overline{\mathbf{f}} \cdot \mathbf{f}(p)=\|\mathbf{f}(p)\|^{2}=\left\|\frac{\mathbf{p}}{\|\mathbf{p}\|}\right\|^{2}\|\mathbf{f}(p)\|^{2} \geq\left|\frac{\mathbf{p}}{\|\mathbf{p}\|} \cdot \mathbf{f}(p)\right|^{2}=\left|f_{0}(p)\right|^{2}
$$

and so $K(f, f) \geq 0$ for all $f \in \mathfrak{p}$.

Let $K(f, f)=0$ for $f \in \mathfrak{p}$, then since by the preceding the integrand in Eqn. (18) is positive, we conclude that $\|\mathbf{f}(p)\|=|\mathbf{p} \cdot \mathbf{f}(p)| /\|\mathbf{p}\|$ on $C_{+}$. Thus $\mathbf{f}(p)$ must be parallel to $\mathbf{p}$, i.e. $\mathbf{f}(p)=\mathbf{p} h(p)$ for some $h$. Since $f_{0}(p)=\mathbf{p} \cdot \mathbf{f}(p) /\|\mathbf{p}\|=\|\mathbf{p}\| h(p)=p_{0} h(p)$ we conclude $f_{\mu}(p)=p_{\mu} h(p)$, i.e. $f \in \mathfrak{p}_{0}$ by Proposition 5.12. Thus $\operatorname{Ker}(K \uparrow \mathfrak{p}) \subseteq \mathfrak{p}_{0}$. The reverse inclusion is obvious.

This establishes the positivity of the IIP on the one-particle space of $\mathcal{H}^{\prime}$, and then the positivity on all of $\mathcal{H}^{\prime}=\mathfrak{F}_{0}(\mathbb{C} \cdot \mathfrak{p})$ follows from the usual arguments for tensor products.

Next, in the usual theory one factors out the zero norm part of $\mathcal{H}^{\prime}$, i.e. $\mathcal{H}^{\prime \prime}:=\operatorname{Ker}\left(\langle\cdot, \cdot\rangle \uparrow \mathcal{H}^{\prime}\right)$.

\subsection{Proposition}

$$
\text { (i) } \mathcal{H}^{\prime \prime}=\left\{\psi \in \mathfrak{F}_{0}(\mathbb{C} \cdot \mathfrak{p}) \mid \psi^{(n)} \in S_{n}\left(\mathfrak{p}_{0} \otimes \mathfrak{p} \otimes \cdots \otimes \mathfrak{p}\right)\right\}
$$

where $\psi^{(n)}$ denotes the $n$-particle component of $\psi$.

(ii) $\mathcal{H}^{\prime} / \mathcal{H}^{\prime \prime}=\mathfrak{F}_{0}\left(\mathbb{C} \cdot \mathfrak{p} / \mathfrak{p}_{0}\right) \cong \mathcal{H}_{\text {phys }}$ where the identification is via the factor map.

Proof: (i) Since $\langle\cdot, \cdot\rangle$ is a positive form on $\mathcal{H}^{\prime}$, the Cauchy-Schwartz inequality applies, hence $\psi \in \mathcal{H}^{\prime \prime}$ iff $\langle\psi, \varphi\rangle=0$ for all $\varphi \in \mathcal{H}^{\prime}$. Let $\psi \in \mathcal{H}^{\prime \prime}$ be given by Eqn. (17), then we have

$$
\begin{aligned}
0 & =\left\langle\sum_{k=1}^{N} S_{n} f_{k 1} \otimes \cdots \otimes f_{k n}, S_{n} g_{1} \otimes \cdots \otimes g_{n}\right\rangle \\
& =\sum_{k=1}^{N} \sum_{\sigma \in P_{n}} \frac{1}{n !}\left\langle f_{k 1}, g_{\sigma(1)}\right\rangle \cdots\left\langle f_{k n}, g_{\sigma(n)}\right\rangle
\end{aligned}
$$

for all $g_{i} \in \mathfrak{p}$. By letting $g_{i}$ vary over all $\mathfrak{p}$, we conclude for each $k$ there is an $i$ such that $\left\langle f_{k i}, g\right\rangle=0$ for all $g \in \mathfrak{p}$, so $f_{k i} \in \mathfrak{p}_{0}$ by Proposition 5.20. This establishes the claim in (i).

(ii) It suffices to examine the $\mathrm{n}$-particle spaces independently, and to ignore the symmetrisation because it creates symmetric sums in which we can examine each term independently. We first examine elementary tensors where no factor is in $\mathfrak{p}_{0}$ (otherwise it is in $\mathcal{H}^{\prime \prime}$ already). Let $\psi=$ 
$f_{1} \otimes \cdots \otimes f_{n}$. Now the factor map comes from the equivalence $\psi \equiv \phi$ iff $\psi-\phi \in \mathcal{H}^{\prime \prime}$, for $\psi, \phi \in \mathcal{H}^{\prime}$ and so we will show the equivalence class $[\psi]$ depends only on the equivalence classes $\left[f_{i}\right]$ in $\mathfrak{p} / \mathfrak{p}_{0}$. To generate equivalent elements in the $i$-th slot, we just add an $f_{1} \otimes \cdots \otimes f_{i-1} \otimes g_{i} \otimes f_{i+1} \cdots f_{n} \in \mathcal{H}^{\prime \prime}$ with $g_{i} \in \mathfrak{p}_{0}$. By doing this for all slots, we have demonstrated for the elementary tensors that the factor map takes $\psi=f_{1} \otimes \cdots \otimes f_{n} \in \mathcal{H}^{\prime}$ to $[\psi]=\left[f_{1}\right] \otimes \cdots \otimes\left[f_{n}\right]$. Extend by linearity to conclude that $\mathcal{H}^{\prime} / \mathcal{H}^{\prime \prime}=\mathfrak{F}_{0}\left(\mathbb{C} \cdot \mathfrak{p} / \mathfrak{p}_{0}\right)$.

Recall from Remark 5.13(ii) that the space $\mathfrak{f}$ of smearing functions corresponding to the lhs of the Maxwell equations are in $\mathfrak{p}_{0}$ and so as it is obvious that $A\left(\mathfrak{p}_{0}\right) \mathcal{H}^{\prime} \subset \mathcal{H}^{\prime \prime}$ from the above characterisations, this substantiates the heuristic claim that the Maxwell equations hold on $\mathcal{H}^{\prime} / \mathcal{H}^{\prime \prime}$.

Considering now the Poincaré transformations, recall we have the symplectic action on $\mathfrak{Y}$ :

$$
\left(V_{g} f\right)(p):=e^{-i p a} \Lambda f\left(\Lambda^{-1} p\right) \quad \forall f \in \mathcal{S}\left(\mathbb{R}^{4}, \mathbb{C}^{4}\right), g=(\Lambda, a) \in \mathcal{P}_{+}^{\uparrow} .
$$

In fact, it is also $K$-unitary because

$$
\begin{aligned}
K\left(V_{g} f, V_{g} h\right) & =-2 \pi \int_{C_{+}}\left(\overline{V_{g} f}\right)^{\mu}(p)\left(V_{g} h\right)_{\mu}(p) \frac{d^{3} p}{p_{0}} \\
& =-2 \pi \int_{C_{+}}(\Lambda \bar{f})^{\mu}\left(\Lambda^{-1} p\right)(\Lambda h)_{\mu}\left(\Lambda^{-1} p\right) \frac{d^{3} p}{p_{0}} \\
& =-2 \pi \int_{C_{+}} \bar{f}^{\mu}\left(\Lambda^{-1} p\right) h_{\mu}\left(\Lambda^{-1} p\right) \frac{d^{3} p}{p_{0}}=K(f, h)
\end{aligned}
$$

since the measure $d^{3} p / p_{0}$ is Lorentz invariant on the light cone. So, using Mintchev [34] again, the second quantized operator $\Gamma\left(V_{g}\right)$ is well-defined on $\mathfrak{F}_{0}(\mathfrak{Y})$, it is $\langle\cdot, \cdot\rangle$-unitary, and it implements $V_{g}$ on $A(f)$. To see that $\Gamma\left(V_{g}\right)$ preserves $\mathcal{H}^{\prime}$, it suffices to note that $V_{g} \mathfrak{p} \subset \mathfrak{p}$ because

$$
p^{\mu}\left(V_{g} f\right)_{\mu}(p)=p^{\mu}\left(\Lambda f\left(\Lambda^{-1} p\right)\right)_{\mu} e^{-i p \cdot a}=\left(\Lambda^{-1} p\right)^{\mu} f_{\mu}\left(\Lambda^{-1} p\right) e^{-i p \cdot a}=0
$$

for $f \in \mathfrak{p}$. Moreover $\Gamma\left(V_{g}\right)$ preserves $\mathcal{H}^{\prime \prime}$ because it preserves both $\mathfrak{p}$ and $\mathfrak{p}_{0}$ where the latter follows from the fact that $\mathfrak{p}_{0}$ is the kernel of the symplectic form on $\mathfrak{p}$ and $\Gamma\left(V_{g}\right)$ is a symplectic transformation. Thus $\Gamma\left(V_{g}\right)$ factors through to $\mathcal{H}^{\prime} / \mathcal{H}^{\prime \prime}=\mathcal{H}_{\text {phys }}$ and in fact, since the IIP now is a Hilbert inner product on this space, the factored $\Gamma\left(V_{g}\right)$ becomes a unitary operator, which will extend to the Hilbert closure of $\mathcal{H}^{\prime} / \mathcal{H}^{\prime \prime}$. It obviously will still implement the (factored through) Poincaré transformations $\widetilde{V}_{g}$ on the factored field operators obtained by restricting $A(\mathfrak{p})$ to $\mathcal{H}^{\prime}$ and then factoring to $\mathcal{H}^{\prime} / \mathcal{H}^{\prime \prime}$.

Returning now to the $\mathrm{C}^{*}$-theory, observe from the characterisations of $\mathcal{H}^{\prime}$ and $\mathcal{H}^{\prime \prime}$ that $\gamma\left(\delta_{\mathfrak{p}}\right)$ will map $\mathcal{H}^{\prime}$ to its Hilbert space closure in $\mathfrak{F}(\mathfrak{Y})$, and will map $\mathcal{H}^{\prime \prime}$ to its closure. (Also note that $\gamma\left(\delta_{\mathfrak{p}_{0}}\right)-\mathbb{1}$ will map $\mathcal{H}^{\prime}$ to the closure of $\mathcal{H}^{\prime \prime}$.) Thus $\gamma\left(\delta_{\mathfrak{p}}\right)$ will lift to operators on the space $\overline{\mathcal{H}^{\prime}} / \overline{\mathcal{H}^{\prime \prime}}$ with $\mathcal{H}^{\prime} / \mathcal{H}^{\prime \prime}$ in their domains. Equipping $\mathcal{H}^{\prime} / \mathcal{H}^{\prime \prime}$ with the inner product coming from the initial IIP, will make these operators into unitaries which extend to the closure $\overline{\mathcal{H}}_{\text {phys }}$ of $\mathcal{H}^{\prime} / \mathcal{H}^{\prime \prime}$. The step of factoring through the operators from $\mathcal{H}^{\prime}$ to $\mathcal{H}^{\prime} / \mathcal{H}^{\prime \prime}$ will identify $\gamma\left(\delta_{\mathfrak{p}_{0}}\right)$ with 11. Thus we obtain an actual Hilbert space representation $\widetilde{\gamma}: \overline{\Delta\left(\mathfrak{p} / \mathfrak{p}_{0}, \widetilde{B}\right)} \rightarrow \mathcal{B}\left(\overline{\mathcal{H}}_{\text {phys }}\right)$. (Recall this last CCR-algebra was our final quasi-local physical algebra $\widetilde{\mathcal{R}}_{0}$ of before). We know this representation is a Fock representation, but this is also clear from

$$
\begin{aligned}
\omega_{0}\left(\delta_{f}\right) & :=\left\langle\Omega, \gamma\left(\delta_{f}\right) \Omega\right\rangle=\lim _{N \rightarrow \infty} \sum_{k=0}^{N} \frac{i^{k}}{k !}\left\langle\Omega,[A(f)]^{k} \Omega\right\rangle \\
& =\exp (-K(f, f) / 4)
\end{aligned}
$$


and the fact that $K(\cdot, \cdot)$ is positive on $\mathfrak{p}$ with kernel $\mathfrak{p}_{0}$. (The usual calculation still works for the last equality). This state $\omega_{0}$ thus extends from $\Delta\left(\mathfrak{p} / \mathfrak{p}_{0}, \widetilde{B}\right)$ to $\overline{\Delta\left(\mathfrak{p} / \mathfrak{p}_{0}, \widetilde{B}\right)}$. In terms of the original $\mathrm{C}^{*}$-algebra, note that $\omega_{0}$ comes from a (nonunique) state $\widetilde{\omega}_{0}$ on $\mathcal{F}_{e}$, because the formula in Eqn. (19) still defines a state on $C^{*}\left(\delta_{\mathfrak{p}}\right)$ by positivity of $K\left\lceil\mathfrak{p}\right.$ (which obviously becomes $\omega_{0}$ after constraining out $\delta_{\mathfrak{p}_{0}}$ ) and it extends by the Hahn-Banach theorem to a state $\widetilde{\omega}_{0}$ on $\mathcal{F}_{e}$. However, $\widetilde{\omega}_{0}$ must necessarily be nonregular which we see as follows. We have $\widetilde{\omega}_{0}\left(\delta_{\mathfrak{p}_{0}}\right)=1$, hence for $c \in \mathfrak{p}_{0}$ and any $f$ with $B(f, c) \neq 0$ :

$$
2 \widetilde{\omega}_{0}\left(\delta_{f}\right)=\widetilde{\omega}_{0}\left(\delta_{f} \delta_{t c}+\delta_{t c} \delta_{f}\right)=2 \widetilde{\omega}_{0}\left(\delta_{f+t c}\right) \cos [t B(f, c) / 2]
$$

for all $t \in \mathbb{R}$. This implies $\widetilde{\omega}_{0}\left(\delta_{f}\right)=0$ and thus the map $t \rightarrow \widetilde{\omega}_{0}\left(\delta_{t f}\right)$ cannot be continuous at $t=0$.

This shows there are two ways of obtaining the final physical algebra, first, we can use Krein representations as studied in this subsection, but these contain pathologies (only dense *-subalgebras are represented, and these as unbounded operators), or second, we can use nonregular representations - which can still produce regular representations on the final physical algebra - and now the operator theory is much better understood. Nonregular representations avoid the problems spelled out by Strocchi's theorems [17, 18] because due to the nonregularity, one cannot use Stone's theorem to obtain generators for the one-parameter groups, hence the operators representing the vector potential do not exist here. This dichotomy between nonregular representations and IIP-representations was pointed out in previous papers, [8, 33].

\section{$6 \quad$ Further topics}

\subsection{Global vs local constraining}

For a system of local constraints $\Theta \rightarrow(\mathcal{F}(\Theta), \mathcal{U}(\Theta))$ as in Definition 3.3, a natural question to ask is the following. What is the relation between the limit algebra $\mathcal{R}_{0}:=\lim \mathcal{R}(\Theta)$ and the algebra $\mathcal{R}_{e}$ obtained from enforcing the full constraint set $\underset{\Theta \in \Gamma}{\cup} \mathcal{U}(\Theta)=: \mathcal{U}_{e}$ in the quasi-local algebra $\mathcal{F}_{0}$ ? In particular, when will $\mathcal{R}_{0}=\mathcal{R}_{e}$ ? In other words, we compare the local constrainings of the net to a single global constraining. (This has bearing on the BRST-constraining algorithm).

Now $\mathcal{R}_{e}=\mathcal{O}_{e} / \mathcal{D}_{e}$ where as usual we have $\mathcal{D}_{e}=\left[\mathcal{F}_{0}\left(\mathcal{U}_{e}-\mathbb{1}\right)\right] \cap\left[\left(\mathcal{U}_{e}-\mathbb{1}\right) \mathcal{F}_{0}\right]$ and $\mathcal{O}_{e}=$ $\left\{F \in \mathcal{F}_{0} \mid[F, D] \in \mathcal{D}_{e} \forall D \in \mathcal{D}_{e}\right\}$.

6.1 Theorem Let the system of local constraints $\Theta \rightarrow(\mathcal{F}(\Theta), \mathcal{U}(\Theta))$ have reduction isotony, then $\mathcal{R}_{0}:=\lim \mathcal{R}(\Theta)=\mathcal{O}_{0} /\left(\mathcal{D}_{e} \cap \mathcal{O}_{0}\right)$ where $\mathcal{O}_{0}:=\lim \mathcal{O}(\Theta)$. Moreover, there is an injective homomorphism of $\mathcal{R}_{0}$ into $\mathcal{R}_{e}$.

Proof: First observe that $\mathcal{D}_{e} \cap \mathcal{O}_{0}=\left[\mathcal{O}_{0}\left(\mathcal{U}_{e}-\mathbb{1}\right)\right] \cap\left[\left(\mathcal{U}_{e}-\mathbb{1}\right) \mathcal{O}_{0}\right]$ because $\mathcal{U}_{e} \subset \mathcal{O}_{0}$ hence every Dirac state on $\mathcal{O}_{0}$ extends to one on $\mathcal{F}_{0}$, and the $\mathcal{D}$-algebra is characterised as the maximal $\mathrm{C}^{*}$-algebra in the kernels of all the Dirac states.

Now denote by $\xi_{\Theta}: \mathcal{O}(\Theta) \rightarrow \mathcal{R}(\Theta)$ the factoring map by $\mathcal{D}(\Theta)$. Let $\Theta_{1} \subseteq \Theta_{2}$, then the diagram

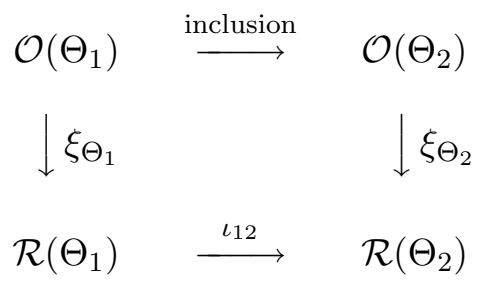


commutes by reduction isotony and the proof of Lemma 3.6. Thus there exists a surjective homomorphism for the inductive limit algebras: $\xi_{0}: \mathcal{O}_{0} \rightarrow \mathcal{R}_{0}$, such that $\xi_{0} \mid \mathcal{O}(\Theta)=\xi_{\Theta}, \Theta \in \Gamma$. Clearly $\mathcal{D}(\Theta) \subset \operatorname{Ker} \xi_{0}$ for all $\Theta \in \Gamma$, hence $\mathcal{U}_{e}-\mathbb{1} \subset \operatorname{Ker} \xi_{0}$, and so by the previous paragraph $\mathcal{D}_{e} \cap \mathcal{O}_{0} \subseteq \operatorname{Ker} \xi_{0}$. Thus $\mathcal{D}_{e} \cap \mathcal{O}(\Theta) \subseteq \operatorname{Ker} \xi_{0} \cap \mathcal{O}(\Theta)=\mathcal{D}(\Theta)$. Since $\mathcal{D}(\Theta) \subset \mathcal{D}_{e}$ we conclude $\mathcal{D}_{e} \cap \mathcal{O}(\Theta)=\mathcal{D}(\Theta)$, and so the global factoring map $\mathcal{O}_{0} \rightarrow \mathcal{O}_{0} /\left(\mathcal{O}_{0} \cap \mathcal{D}_{e}\right)$ coincides on each $\mathcal{O}(\Theta)$ with $\xi_{\Theta}$. Thus it is $\xi_{0}$, i.e. $\operatorname{Ker} \xi_{0}=\mathcal{D}_{e} \cap \mathcal{O}_{0}$, so $\mathcal{R}_{0}=\xi_{0}\left(\mathcal{O}_{0}\right)=\mathcal{O}_{0} /\left(\mathcal{O}_{0} \cap \mathcal{D}_{e}\right)$.

To prove the last claim, we just apply Lemma 3.6 to the pair $\left(\mathcal{O}_{0}, \mathcal{U}_{e}\right) \subset\left(\mathcal{F}_{0}, \mathcal{U}_{e}\right)$. To verify its two conditions, note that we already know by the first part of the proof that $\mathcal{D}_{e} \cap \mathcal{O}_{0}=$ $\left[\mathcal{O}_{0}\left(\mathcal{U}_{e}-\mathbb{1}\right)\right] \cap\left[\left(\mathcal{U}_{e}-\mathbb{1}\right) \mathcal{O}_{0}\right]$ so we only need to check the second condition. We also saw above that $\mathcal{D}_{e} \cap \mathcal{O}_{0}$ is an ideal in $\mathcal{O}_{0}$ (the kernel of a homomorphism), hence the algebra of observables in $\mathcal{O}_{0}$ of the constraints $\mathcal{U}_{e}$ is all of $\mathcal{O}_{0}$ (using Theorem 2.3(ii)). Thus we only need to show that $\mathcal{O}_{0} \subset \mathcal{O}_{e}$, i.e. that $\mathcal{O}(\Theta) \subset \mathcal{O}_{e}$ for all $\Theta \in \Gamma$. By Theorem 2.3(iii) we only need to show that $\left[F, \mathcal{U}_{e}\right] \subset \mathcal{D}_{e} \forall F \in \mathcal{O}(\Theta)$, but this follows immediately from the last paragraph since for an observable $F \in \mathcal{O}_{0}$ we have: $\left[F, \mathcal{U}_{e}\right] \subset \mathcal{D}_{e} \cap \mathcal{O}_{0} \subset \mathcal{D}_{e}$.

We do not as yet have useful general criteria to ensure that $\mathcal{R}_{0}=\mathcal{R}_{e}$, though we now verify that it holds for both stages of constraining in the Gupta-Bleuler example.

Example. Recall the first stage of constraining in the previous example. We had a system of local constraints $\Gamma \ni \Theta \rightarrow(\mathcal{F}(\Theta), \mathcal{U}(\Theta))$ where $\mathcal{F}(\Theta)=\mathrm{C}^{*}\left(\delta_{\mathfrak{X}(\Theta)} \cup \mathcal{U}(\Theta)\right)$ and $\mathcal{U}(\Theta)=$ $\left\{U_{T_{h}} \mid h \in C_{c}^{\infty}\left(\Theta, \mathbb{R}^{4}\right)\right\}$. By Theorem 5.5 we have $\mathcal{R}(\Theta) \cong \mathrm{C}^{*}\left(\delta_{\mathfrak{p}(\Theta)}\right)$ and so $\mathcal{R}_{0}=\underline{\lim } \mathcal{R}(\Theta)=$ $\overline{\Delta(\mathfrak{p}, B)}$ where $\mathfrak{p}=\bigcup_{\Theta \in \Gamma} \mathfrak{p}(\Theta)$. We need to compare this to $\mathcal{R}_{e}$ which we obtain from the system $\left(\mathcal{F}_{0}, \mathcal{U}_{e}\right)$ where $\mathcal{F}_{0}=\underset{\lim }{\longrightarrow} \mathcal{F}(\Theta)=\mathrm{C}^{*}\left(\mathcal{U}_{e} \cup \delta_{\mathfrak{Z}_{(0)}}\right)$, (cf. Remark 5.4 (i)) and $\mathcal{U}_{e}=\underset{\Theta \in \Gamma}{\cup} \mathcal{U}(\Theta)=\mathcal{U}_{(0)}$. Now the method in the proof of Theorem 5.5 did not use the assumption $\Theta \in \Gamma$, hence it can be transcribed to prove that $\mathcal{O}_{e}=\mathrm{C}^{*}\left(\delta_{\mathfrak{p}_{e}}\right)+\mathcal{D}_{e}$ where $\mathfrak{p}_{e}:=\left\{f \in \mathfrak{Z}_{(0)} \mid T_{h}(f)=f \forall h \in\right.$ $\left.C_{c}^{\infty}(\Theta, \mathbb{R}), \Theta \in \Gamma\right\}$. Each $f \in \mathfrak{p}_{e} \subset \mathfrak{Z}_{(0)}$ is in some $\mathfrak{Z}(\Theta)$, so is in $\mathfrak{p}(\Theta)$ by the defining condition.

Thus $\mathfrak{p}_{e} \cap \mathfrak{Z}(\Theta) \subseteq \mathfrak{p}(\Theta)$. However, by Theorem 5.5, these are characterised by $p_{\mu} f^{\mu} \uparrow C_{+}=0$, and by Eqn. (8) this implies $T_{h}(f)=f$ for all $h \in C_{c}^{\infty}\left(\mathbb{R}^{4}, \mathbb{R}\right)$. Thus $\mathfrak{p}_{e}=\mathfrak{p}$, so $\mathcal{O}_{e}=\mathrm{C}^{*}\left(\delta_{\mathfrak{p}}\right)+\mathcal{D}_{e}$ and hence by the argument in the last part of the proof of Theorem 5.5 we have $\mathcal{R}_{e} \cong \mathrm{C}^{*}\left(\delta_{\mathfrak{p}}\right) \cong \mathcal{R}_{0}$.

Next we verify for the second stage of constraining that the local and global constrainings ultimately coincide. Here we have the system of local constraints: $\Gamma \ni \Theta \rightarrow(\mathcal{R}(\Theta), \widetilde{\mathcal{U}}(\Theta))$ where $\mathcal{R}(\Theta)=\overline{\Delta(\mathfrak{p}(\Theta), B)}$ and $\widetilde{\mathcal{U}}(\Theta)=\delta_{\mathfrak{s}(\Theta)}, \mathfrak{s}(\Theta)=\mathfrak{p}_{0} \cap \mathfrak{p}(\Theta)$. By Theorem 5.14 we have $\widetilde{\mathcal{R}}_{0} \cong \overline{\Delta\left(\mathfrak{p} / \mathfrak{p}_{0}, \widetilde{B}\right)}$. We need to compare this to the physical algebra $\widetilde{\mathcal{R}}_{e}$ obtained from the system $\left(\mathcal{R}_{0}, \widetilde{\mathcal{U}}_{e}\right)$ where $\mathcal{R}_{0}=\underline{\lim } \mathcal{R}(\Theta)=\overline{\Delta(\mathfrak{p}, B)}$ and $\widetilde{\mathcal{U}}_{e}=\underset{\Theta \in \Gamma}{\cup} \widetilde{\mathcal{U}}(\Theta)=\delta_{\mathfrak{p}_{0}}$. By Theorem 5.2 in 8 we have $\widetilde{\mathcal{R}}_{e} \cong \overline{\Delta\left(\mathfrak{p} / \mathfrak{p}_{0}, \widetilde{B}\right)} \cong \widetilde{\mathcal{R}_{0}}$, and this proves the claim.

\subsection{Reduction by stages}

In this subsection we address the problem of reduction by stages, i.e. subdivide the initial constraint set, then impose these constraint sets along an increasing chain (terminating with the full set of constraints), and analyse when the final physical algebra of the chain is the same as that obtained from a single constraining by the full set. This problem occurred in the Gupta-Bleuler example, and is related also to the one in the previous subsection.

6.2 Definition An $\mathbf{n}$-chain of constraints consists of a first-class constraint system $(\mathcal{F}, \mathcal{C})$ and a chain of subsets

$$
\{0\} \neq \mathcal{C}_{1} \subset \mathcal{C}_{2} \subset \cdots \subset \mathcal{C}_{n}=\mathcal{C}
$$

such that $\mathcal{C} \subset \mathcal{O}_{i} \forall i=1,2, \ldots, n$, where we henceforth denote by $\left(\mathfrak{S}_{D_{i}}, \mathcal{D}_{i}, \mathcal{O}_{i}, \mathcal{R}_{i}, \xi_{i}\right)$ the data resulting from application of a T-procedure to $\left(\mathcal{F}, \mathcal{C}_{i}\right)$. (Recall that $\xi_{i}: \mathcal{O}_{i} \rightarrow \mathcal{R}_{i}$ denotes the canonical factorization map). By convention we will omit the subscript $i$ when $i=n$. 
Note that $\mathfrak{S}_{D}=\mathfrak{S}_{D_{n}} \subset \mathfrak{S}_{D_{n-1}} \subset \cdots \subset \mathfrak{S}_{D_{2}} \subset \mathfrak{S}_{D_{1}}$ and $\mathcal{D}_{1} \subset \mathcal{D}_{2} \subset \cdots \subset \mathcal{D}_{n}=\mathcal{D}$. The condition $\mathcal{C} \subset \mathcal{O}_{i}$ is nontrivial, but necessary for the procedure in the next theorem. Below we will use subscript notation $A_{i}, A_{(i)}$ and $A_{\{i\}}$ to distinguish between similar objects in different contexts.

6.3 Theorem Given an n-chain of constraints as above, we define inductively the following cascade of first-class constraint systems $\left(\mathcal{R}_{(k-1)}, \xi_{\{k-1\}}\left(\mathcal{C}_{k}\right)\right), k=1, \ldots, n$ with T-procedure data $\left(\mathfrak{S}_{D(k)}, \mathcal{D}_{(k)}, \mathcal{O}_{(k)}, \mathcal{R}_{(k)}, \xi_{(k)}\right)$ and notation $\xi_{\{k\}}:=\xi_{(k)} \circ \xi_{(k-1)} \circ \cdots \circ \xi_{(1)}$ and conventions $\xi_{\{0\}}:=\mathrm{id}, \mathcal{R}_{(0)}=\mathcal{F}$. Then

(i) $\operatorname{Dom} \xi_{\{k\}}=\mathcal{O}_{1} \cap \mathcal{O}_{2} \cap \cdots \cap \mathcal{O}_{k}=: \mathcal{O}_{\{k\}}$, Ker $\xi_{\{k\}}=\mathcal{O}_{\{k\}} \cap \mathcal{D}_{k}$, and $\operatorname{Ran} \xi_{\{k\}}=\mathcal{R}_{(k)}$ where we use the conventions $\mathcal{O}_{\{0\}}:=\mathcal{F}$ and $\mathcal{D}_{0}=\{0\}$.

(ii) $\mathcal{D}_{(k)}=\xi_{\{k-1\}}\left(\mathcal{O}_{\{k-1\}} \cap \mathcal{D}_{k}\right)$,

(iii) $\mathcal{O}_{(k)}=\xi_{\{k-1\}}\left(\mathcal{O}_{\{k\}}\right)$,

(iv) $\mathcal{R}_{(k)} \cong \mathcal{O}_{\{k\}} /\left(\mathcal{O}_{\{k-1\}} \cap \mathcal{D}_{k}\right) \subset \mathcal{R}_{k}$,

(v) the map $\varphi_{k}: \mathfrak{S}_{D_{k}} \uparrow \mathcal{O}_{\{k\}} \rightarrow \mathfrak{S}\left(\mathcal{R}_{(k)}\right)$ defined by $\varphi_{k}(\omega)\left(\xi_{\{k\}}(A)\right):=\omega(A), A \in \mathcal{O}_{\{k\}}$, gives a bijection $\varphi_{k}: \mathfrak{S}_{D_{k+1}} \uparrow \mathcal{O}_{\{k\}} \rightarrow \mathfrak{S}_{D(k+1)}$.

We will call the application of a T-procedure to $\left(\mathcal{R}_{(k-1)}, \xi_{\{k-1\}}\left(\mathcal{C}_{k}\right)\right), k=1, \ldots, n$ to produce the data $\left(\mathfrak{S}_{D(k)}, \mathcal{D}_{(k)}, \mathcal{O}_{(k)}, \mathcal{R}_{(k)}, \xi_{(k)}\right)$ the $k^{\text {th }}$ stage reduction of the given n-chain.

Proof: We apply the second principle of induction, and also remind the reader that $\mathcal{C} \subset \mathcal{O}_{i}$ $\forall i$. For $k=1$ we have by convention that $\left(\mathcal{R}_{(0)}, \xi_{\{0\}}\left(\mathcal{C}_{1}\right)\right)=\left(\mathcal{F}, \mathcal{C}_{1}\right)$ which is first-class, and $\left(\mathfrak{S}_{D(1)}, \mathcal{D}_{(1)}, \mathcal{O}_{(1)}, \mathcal{R}_{(1)}, \xi_{(1)}\right)=\left(\mathfrak{S}_{D_{1}}, \mathcal{D}_{1}, \mathcal{O}_{1}, \mathcal{R}_{1}, \xi_{1}\right)$. Thus Dom $\xi_{\{1\}}=\operatorname{Dom} \xi_{1}=\mathcal{O}_{1}=\mathcal{O}_{\{1\}}$, $\operatorname{Ker} \xi_{\{1\}}=\operatorname{Ker} \xi_{1}=\mathcal{D}_{1}=\mathcal{O}_{\{1\}} \cap \mathcal{D}_{1}, \operatorname{Ran} \xi_{\{1\}}=\operatorname{Ran} \xi_{1}=\mathcal{R}_{1}=\mathcal{R}_{(1)}$. Moreover $\mathcal{D}_{(1)}=$ $\mathcal{D}_{1}=\xi_{\{0\}}\left(\mathcal{O}_{\{0\}} \cap \mathcal{D}_{1}\right), \mathcal{O}_{(1)}=\xi_{\{0\}}\left(\mathcal{O}_{\{1\}}\right)=\mathcal{O}_{1}$ and $\mathcal{R}_{(1)}=\mathcal{R}_{1}=\mathcal{O}_{\{1\}} /\left(\mathcal{O}_{\{0\}} \cap \mathcal{D}_{1}\right)$. Now using $\mathcal{C}_{2} \subset \mathcal{C} \subset \mathcal{O}_{1}$, the bijection $\varphi_{1}: \mathfrak{S}_{D_{1}}\left\lceil\mathcal{O}_{1} \rightarrow \mathfrak{S}\left(\mathcal{R}_{1}\right)\right.$ given by Theorem 2.4, produces for $\omega \in \mathfrak{S}_{D_{2}}$ :

$$
\varphi_{1}(\omega)\left(\xi_{1}\left(C^{*} C\right)\right)=\omega\left(C^{*} C\right)=0=\omega\left(C C^{*}\right)=\varphi_{1}(\omega)\left(\xi_{1}\left(C C^{*}\right)\right)
$$

for all $C \in \mathcal{C}_{2}$, i.e. $\varphi_{1}(\omega) \in \mathfrak{S}_{D(2)}$. Conversely, if $\varphi_{1}(\omega) \in \mathfrak{S}_{D(2)}$ then $\omega \in \mathfrak{S}_{D_{2}}$. Thus the theorem holds for $k=1$.

For the induction step, fix an integer $m \geq 1$ and assume the theorem is true for all $k \leq$ $m$. We prove that it holds for $m+1$. Now $\left(\mathcal{R}_{(m)}, \xi_{\{m\}}\left(\mathcal{C}_{m+1}\right)\right)$ is first-class, because by (v), $\varphi_{m}\left(\mathfrak{S}_{D_{m+1}} \uparrow \mathcal{O}_{\{m\}}\right)=\mathfrak{S}_{D(m+1)} \neq \emptyset$ since by $\mathcal{C}_{m+1} \subset \mathcal{C} \subset \mathcal{O}_{i}$ we have $\emptyset \neq \mathfrak{S}_{D} \mid \mathcal{O}_{\{m\}}$ and $\varphi_{m}$ is a bijection. We first prove (ii).

$$
\begin{aligned}
& \mathcal{D}_{(m+1)}=\left\{\xi_{\{m\}}(F) \mid F \in \mathcal{O}_{\{m\}} \quad\right. \text { and } \\
&\left.\omega\left(\xi_{\{m\}}\left(F^{*} F\right)\right)=0=\omega\left(\xi_{\{m\}}\left(F F^{*}\right)\right) \forall \omega \in \mathfrak{S}_{D(m+1)}\right\} \\
&=\left\{\xi_{\{m\}}(F) \mid F \in \mathcal{O}_{\{m\}}\right. \text { and } \\
&\left.\widehat{\omega}\left(F^{*} F\right)=0=\widehat{\omega}\left(F F^{*}\right) \forall \widehat{\omega} \in \mathfrak{S}_{D_{m+1}}\right\} \\
& \\
& \quad(\text { using }(\mathrm{v}) \text { of induction assumption }) \\
&= \xi_{\{m\}}\left(\mathcal{D}_{m+1} \cap \mathcal{O}_{\{m\}}\right) .
\end{aligned}
$$

For (iii) we see:

$$
\mathcal{O}_{(m+1)}=\left\{\xi_{\{m\}}(F) \mid F \in \mathcal{O}_{\{m\}} \quad\right. \text { and }
$$




$$
\begin{gathered}
\left.\left[\xi_{\{m\}}(F), \xi_{\{m\}}\left(\mathcal{C}_{m+1}\right)\right] \subset \mathcal{D}_{(m+1)}\right\}(\text { by Theorem 2.3(iii)) } \\
=\left\{\xi_{\{m\}}(F) \mid F \in \mathcal{O}_{\{m\}}\right. \text { and } \\
\left.\quad \xi_{\{m\}}\left(\left[F, \mathcal{C}_{m+1}\right]\right) \subset \xi_{\{m\}}\left(\mathcal{D}_{m+1} \cap \mathcal{O}_{\{m\}}\right)\right\} \\
=\left\{\xi_{\{m\}}(F) \mid F \in \mathcal{O}_{\{m\}}\right. \text { and } \\
\left.\left[F, \mathcal{C}_{m+1}\right] \subset \mathcal{D}_{m+1} \cap \mathcal{O}_{\{m\}}+\mathcal{O}_{\{m\}} \cap \mathcal{D}_{m}\right\}
\end{gathered}
$$

Now since $\mathcal{D}_{m} \subset \mathcal{D}_{m+1}$, we have $\mathcal{D}_{m+1} \cap \mathcal{O}_{\{m\}}+\mathcal{O}_{\{m\}} \cap \mathcal{D}_{m}=\mathcal{D}_{m+1} \cap \mathcal{O}_{\{m\}}$ and $\mathcal{C}_{m+1} \subset \mathcal{O}_{\{m\}}$ and so $\left[F, \mathcal{C}_{m+1}\right] \subset \mathcal{O}_{\{m\}}$ for all $F \in \mathcal{O}_{\{m\}}$. Thus

$$
\begin{aligned}
\mathcal{O}_{(m+1)} & =\left\{\xi_{\{m\}}(F) \mid F \in \mathcal{O}_{\{m\}} \quad \text { and } \quad\left[F, \mathcal{C}_{m+1}\right] \subset \mathcal{D}_{m+1}\right\} \\
& =\xi_{\{m\}}\left(\mathcal{O}_{\{m\}} \cap \mathcal{O}_{m+1}\right)=\xi_{\{m\}}\left(\mathcal{O}_{\{m+1\}}\right) .
\end{aligned}
$$

For (iv), note that

$$
\mathcal{R}_{(m+1)}=\mathcal{O}_{(m+1)} / \mathcal{D}_{(m+1)}=\xi_{\{m\}}\left(\mathcal{O}_{\{m+1\}}\right) / \xi_{\{m\}}\left(\mathcal{D}_{m+1} \cap \mathcal{O}_{\{m\}}\right)
$$

Define a map $\psi: \mathcal{R}_{(m+1)} \rightarrow \mathcal{R}_{m+1}$ by

$$
\psi\left(\xi_{\{m\}}(A)+\xi_{\{m\}}\left(\mathcal{D}_{m+1} \cap \mathcal{O}_{\{m\}}\right)\right):=A+\mathcal{D}_{m+1} \quad A \in \mathcal{O}_{\{m+1\}} .
$$

To see that it is well-defined, let $B \in \mathcal{O}_{\{m+1\}}$ be such that $\xi_{\{m\}}(A)-\xi_{\{m\}}(B) \in \xi_{\{m\}}\left(\mathcal{D}_{m+1} \cap\right.$ $\left.\mathcal{O}_{\{m\}}\right)$, i.e. $A-B \in \mathcal{D}_{m+1} \cap \mathcal{O}_{\{m\}}+\mathcal{O}_{\{m\}} \cap \mathcal{D}_{m}=\mathcal{D}_{m+1} \cap \mathcal{O}_{\{m\}}$, and so

$$
\psi\left(\xi_{\{m\}}(B)+\xi_{\{m\}}\left(\mathcal{D}_{m+1} \cap \mathcal{O}_{\{m\}}\right)\right)=B+\mathcal{D}_{m+1}=A+\mathcal{D}_{m+1} .
$$

It is easy to see that $\psi$ is a ${ }^{*}$-homomorphism onto the subalgebra $\mathcal{O}_{\{m+1\}} / \mathcal{D}_{m+1} \cap \mathcal{O}_{\{m\}} \subset$ $\mathcal{R}_{m+1}$ and since $\operatorname{Ker} \psi=\xi_{\{m\}}\left(\mathcal{D}_{m+1} \cap \mathcal{O}_{\{m\}}\right)=\mathcal{D}_{(m+1)}$ which is the zero of $\mathcal{R}_{(m+1)}$, $\psi$ is a monomorphism.

To prove (i), recall that $\xi_{\{m+1\}}=\xi_{(m+1)} \circ \xi_{\{m\}}$, so

$$
\begin{aligned}
\operatorname{Dom} \xi_{\{m+1\}} & =\left\{F \in \operatorname{Dom} \xi_{\{m\}} \mid \xi_{\{m\}}(F) \in \operatorname{Dom} \xi_{(m+1)}=\mathcal{O}_{(m+1)}\right\} \\
& =\left\{F \in \mathcal{O}_{\{m\}} \mid \xi_{\{m\}}(F) \in \xi_{\{m\}}\left(\mathcal{O}_{\{m+1\}}\right)\right\} \\
& =\left\{F \in \mathcal{O}_{\{m\}} \mid F \in \mathcal{O}_{\{m+1\}}+\mathcal{O}_{\{m\}} \cap \mathcal{D}_{m}=\mathcal{O}_{\{m+1\}}\right\}
\end{aligned}
$$

because $\mathcal{D}_{m} \subset \mathcal{D}_{m+1} \subset \mathcal{O}_{m+1}$. Thus Dom $\xi_{\{m+1\}}=\mathcal{O}_{\{m+1\}}$. Now

$$
\begin{aligned}
\operatorname{Ker} \xi_{\{m+1\}} & =\left\{F \in \operatorname{Dom} \xi_{\{m+1\}} \mid \xi_{\{m\}}(F) \in \operatorname{Ker} \xi_{(m+1)}=\mathcal{D}_{(m+1)}\right\} \\
& =\left\{F \in \mathcal{O}_{\{m+1\}} \mid \xi_{\{m\}}(F) \in \xi_{\{m\}}\left(\mathcal{D}_{m+1} \cap \mathcal{O}_{\{m\}}\right)\right\} \\
& =\left\{F \in \mathcal{O}_{\{m+1\}} \mid\right. \\
& \left.\quad F \in \mathcal{D}_{m+1} \cap \mathcal{O}_{\{m\}}+\mathcal{O}_{\{m\}} \cap \mathcal{D}_{m}=\mathcal{D}_{m+1} \cap \mathcal{O}_{\{m\}}\right\} \\
& =\mathcal{O}_{\{m+1\}} \cap \mathcal{D}_{m+1} \cdot \\
\operatorname{Ran} \xi_{\{m+1\}} & =\xi_{(m+1)}\left(\xi_{\{m\}}\left(\operatorname{Dom} \xi_{\{m+1\}}\right)\right)=\xi_{(m+1)}\left(\xi_{\{m\}}\left(\mathcal{O}_{\{m+1\}}\right)\right) \\
& =\xi_{(m+1)}\left(\mathcal{O}_{\{m+1\}}\right)=\mathcal{R}_{(m+1)} .
\end{aligned}
$$


Finally, to prove $(\mathrm{v})$, since $\varphi_{m+1}$ is a surjection, each $\omega \in \mathfrak{S}_{D(m+2)}$ is of the form $\omega=\varphi_{m+1}(\widehat{\omega})$ for some $\widehat{\omega} \in \mathfrak{S}_{D_{m+1}} \uparrow \mathcal{O}_{\{m+1\}}$. Now $\omega \in \mathfrak{S}_{D(m+2)}$ iff $\omega\left(\xi_{\{m+1\}}\left(C^{*} C\right)\right)=0=\omega\left(\xi_{\{m+1\}}\left(C C^{*}\right)\right)$ for all $C \in \mathcal{C}_{m+2}$ iff $\widehat{\omega}\left(C^{*} C\right)=0=\widehat{\omega}\left(C C^{*}\right)$ for all $C \in \mathcal{C}_{m+2}$ iff $\widehat{\omega} \in \mathfrak{S}_{D_{m+2}} \mid \mathcal{O}_{\{m+1\}}$.

Example. The Gupta-Bleuler model of the previous section provide examples of 2-chains of constraints both at the local and the global levels. We will only consider the global level, and refer freely to the example of the last section where both global constrainings were done. Let $\mathcal{C}_{1}:=\left\{U_{T_{h}}-\mathbb{1} \mid h \in C_{c}^{\infty}\left(\mathbb{R}^{4}, \mathbb{R}\right)\right\}$ and let the total constraint set in $\mathcal{F}_{0}$ be $\mathcal{C}=\mathcal{C}_{2}:=\mathcal{C}_{1} \cup \widetilde{\mathcal{C}}$ where $\widetilde{\mathcal{C}}:=\mathbb{1}-\widetilde{\mathcal{U}}_{e}=\left\{\mathbb{1}-\delta_{f} \mid f \in \mathfrak{p}_{0}\right\}$.

6.4 Claim $\mathcal{C}_{1} \subset \mathcal{C}_{2}=\mathcal{C}$ is a 2-chain of constraints in $\mathcal{F}_{0}=\mathrm{C}^{*}\left(\delta_{\mathfrak{Z}_{(0)}} \cup \mathcal{U}_{(0)}\right)$. (Notation as in Remark 5.4 (i)).

Proof: To see that $\mathcal{C}$ is first-class, define a state $\widehat{\omega}$ on $\overline{\Delta\left(\mathfrak{Z}_{(0)}, B\right)} \subset \mathcal{F}_{0}$ by $\widehat{\omega}\left(\delta_{f}\right)=1$ if $f \in \mathfrak{p}_{0}$, and otherwise $\widehat{\omega}\left(\delta_{f}\right)=0$ (that this defines a state is easy to check). Since by Theorem 5.5 the space $\mathfrak{p}$ is pointwise invariant under $T_{h}$ for $h \in C_{c}^{\infty}\left(\mathbb{R}^{4}, \mathbb{R}\right.$ ), (also using Eqn. (8)) so is $\mathfrak{p}_{0}$, hence $\widehat{\omega}$ is invariant under $\mathcal{G}_{(0)}=$ the group generated by $\mathcal{G}(\Theta), \Theta \in \Gamma$. Thus $\widehat{\omega}$ extends (uniquely) to a Dirac state on $\mathcal{F}_{0}$ (by a trivial application of Theorem 4.1). Thus $\mathcal{C}$ is first class.

It is obvious that $\mathcal{C} \subset \mathcal{D}_{2} \subset \mathcal{O}_{2}$, so we only need to show that $\mathcal{C} \subset \mathcal{O}_{1}$. By Theorem 5.5 and the last subsection we have $\mathcal{O}_{1}=\mathrm{C}^{*}\left(\delta_{\mathfrak{p}}\right)+\mathcal{D}_{1}$ and as $\mathcal{C}_{1} \subset \mathcal{D}_{1}$ and $\delta_{\mathfrak{p}_{0}} \subset \mathrm{C}^{*}\left(\delta_{\mathfrak{p}}\right)$ it follows that $\mathcal{C} \subset \mathcal{O}_{1}$.

Now we want to show that $\mathcal{R}_{2}=\mathcal{R}_{(2)}$, i.e. the two-step reduction by stages produces the same physical algebra as a single reduction by the full constraint set $\mathcal{C}$. Recall that by Theorem 6.3(iv), we have a monomorphic imbedding:

$$
\mathcal{R}_{(2)} \cong \mathcal{O}_{\{2\}} /\left(\mathcal{O}_{\{1\}} \cap \mathcal{D}_{2}\right) \subset \mathcal{R}_{2}=\mathcal{O}_{2} / \mathcal{D}_{2}
$$

where $\mathcal{O}_{\{2\}}=\mathcal{O}_{1} \cap \mathcal{O}_{2}, \mathcal{O}_{1}=\mathcal{O}_{\{1\}}$. So we will have the desired isomorphism $\mathcal{R}_{2}=\mathcal{R}_{(2)}$, if we can show that this imbedding is surjective. Now we know from the last subsection that $\mathcal{O}_{1}=C^{*}\left(\delta_{\mathfrak{p}}\right)+\mathcal{D}_{1}$, and below in the next two claims we prove that $\mathcal{O}_{2}=C^{*}\left(\delta_{\mathfrak{p}}\right)+\mathcal{D}_{2}$. Then since $\mathcal{D}_{1} \subset \mathcal{D}_{2}$ we have $\mathcal{O}_{1} \subset \mathcal{O}_{2}$, so

$$
\mathcal{R}_{(2)} \cong \mathcal{O}_{1} /\left(\mathcal{O}_{1} \cap \mathcal{D}_{2}\right) \subset \mathcal{R}_{2}=\mathcal{O}_{2} / \mathcal{D}_{2}
$$

Now note that each equivalence class corresponding to an element of $\mathcal{R}_{2}$ is of the form $A+\mathcal{D}_{2}$ with $A \in C^{*}\left(\delta_{\mathfrak{p}}\right)$, and this contains the equivalence class of $A+\mathcal{D}_{1}$ from $\mathcal{O}_{1} /\left(\mathcal{O}_{1} \cap \mathcal{D}_{2}\right)$. So the imbedding is surjective.

It remains to prove that $\mathcal{O}_{2}=C^{*}\left(\delta_{\mathfrak{p}}\right)+\mathcal{D}_{2}$. We first prove:

\subsection{Claim}

$\mathcal{O}_{2}=\mathrm{C}^{*}\left(\delta_{\mathfrak{p}_{0}^{\prime}}\right)+\mathcal{D}_{2} \quad$ where $\quad \mathfrak{p}_{0}^{\prime}=\left\{f \in \mathfrak{Z}_{(0)} \mid B(f, s)=0 \quad \forall s \in \mathfrak{p}_{0}\right\}$.

Proof: We adapt the proof of Theorem 5.5. Since $\mathcal{O}_{2} \subset \mathcal{F}_{0}=\mathrm{C}^{*}\left(\delta_{\mathfrak{Z}_{(0)}} \cup \mathcal{U}_{(0)}\right)$, for a general $A \in \mathcal{O}_{2}$ we can write

$$
A=\lim _{n \rightarrow \infty} A_{n} \quad \text { where } \quad A_{n}=\sum_{i=1}^{N_{n}} \delta_{f_{i}} \sum_{j=1}^{L_{n}} \lambda_{i j}^{(n)} U_{\gamma_{i j}^{(n)}}
$$

where $f_{i} \neq f_{j}$ if $i \neq j, f_{i} \in \mathfrak{Z}_{(0)}, \gamma_{i j}^{(n)} \in \mathcal{G}_{(0)}$ and $\lambda_{i j}^{(n)} \in \mathbb{C}$. Consider the equivalence classes of $\mathfrak{Z}_{(0)} / \mathfrak{p}_{0}$. If $f_{i}-f_{j}=: s \in \mathfrak{p}_{0}$, then $\delta_{f_{i}}=\delta_{f_{j}} \cdot \delta_{s} \exp \left(i B\left(s, f_{j}\right) / 2\right)$ and $\delta_{s} \in \widetilde{\mathcal{U}}_{e}$. Thus we can write Eqn. (20) in the form

$$
A_{n}=\sum_{i=1}^{N_{n}} \delta_{f_{i}} \sum_{j=1}^{L_{n}} \sum_{k=1}^{K_{n}} \lambda_{i j k}^{(n)} \delta_{s_{i j}} U_{\gamma_{i k}^{(n)}}
$$


where $f_{i}-f_{j} \notin \mathfrak{p}_{0}$ if $i \neq j$ and $s_{i j} \in \mathfrak{p}_{0}$. Let $\omega \in \mathfrak{S}_{D_{2}}$, then

$$
\begin{aligned}
\pi_{\omega}\left(A_{n}\right) \Omega_{\omega} & =\sum_{i=1}^{N_{n}} \zeta_{i}^{(n)} \pi_{\omega}\left(\delta_{f_{i}}\right) \Omega_{\omega} \\
\text { where } \quad \zeta_{i}^{(n)} & =\sum_{j=1}^{L_{n}} \sum_{k=1}^{K_{n}} \lambda_{i j k}^{(n)} \in \mathbb{C} .
\end{aligned}
$$

Let $h \in \mathfrak{p}_{0}$, then from $\delta_{h} \in \widetilde{\mathcal{U}}_{e}$ and $A \in \mathcal{O}_{2}$, we get, using Eqn. (22):

$$
\begin{aligned}
0 & =\omega\left(A^{*}\left(\delta_{h}-\mathbb{1}\right)^{*}\left(\delta_{h}-\mathbb{1}\right) A\right)=\lim _{n \rightarrow \infty} \omega\left(A_{n}^{*}\left(2 \mathbb{1}-\delta_{h}-\delta_{-h}\right) A_{n}\right) \\
& =\lim _{n \rightarrow \infty}\left\{2 \omega\left(A_{n}^{*} A_{n}\right)-\sum_{i, j=1}^{N_{n}} \bar{\zeta}_{i}^{(n)} \zeta_{j}^{(n)} \omega\left(\delta_{-f_{i}}\left(\delta_{h}+\delta_{-h}\right) \delta_{f_{j}}\right)\right\}
\end{aligned}
$$

The state $\widehat{\omega}$ on $\mathcal{F}_{0}$ defined by $\widehat{\omega}\left(\delta_{f}\right):=\chi_{\mathfrak{p}_{0}}(f)$ and $\widehat{\omega}\left(U_{g}\right)=1 \forall g \in \mathcal{G}_{(0)}$ (encountered in the proof of Claim 6.4) is in $\mathfrak{S}_{D_{2}}$, so Eqn. (23) becomes for it:

$$
\begin{aligned}
0 & =\lim _{n \rightarrow \infty}\left\{2 \sum_{j=1}^{N_{n}}\left|\zeta_{j}^{(n)}\right|^{2}-\sum_{j=1}^{N_{n}}\left|\zeta_{j}^{(n)}\right|^{2}\left(e^{i B\left(h, f_{j}\right)}+e^{-i B\left(h, f_{j}\right)}\right)\right\} \\
& =2 \lim _{n \rightarrow \infty} \sum_{j=1}^{N_{n}}\left|\zeta_{j}^{(n)}\right|^{2}\left(1-\cos B\left(h, f_{j}\right)\right)
\end{aligned}
$$

where we made use of $f_{i}-f_{j} \notin \mathfrak{p}_{0}$ if $i \neq j$ and the equation

$$
\begin{aligned}
\pi_{\omega}\left(\delta_{-f_{i}} \delta_{h} \delta_{f_{j}}\right) \Omega_{\omega} & =\pi_{\omega}\left(\delta_{-f_{i}} e^{i B\left(h, f_{j}\right)} \delta_{f_{j}} \delta_{h}\right) \Omega_{\omega} \\
& =e^{i B\left(h, f_{j}\right)} e^{-i B\left(f_{i}, f_{j}\right) / 2} \pi_{\omega}\left(\delta_{f_{j}-f_{i}}\right) \Omega_{\omega}
\end{aligned}
$$

Now the terms in the sum of Eqn. (24) are all positive so in the limit these must individually vanish, i.e.

$$
\lim _{n \rightarrow \infty}\left|\zeta_{j}^{(n)}\right|^{2}\left(1-\cos B\left(h, f_{j}\right)\right)=0 \quad \forall h \in \mathfrak{p}_{0} .
$$

Thus since the first factor is independent of $h$ and the second is independent of $n$, either $\lim _{n \rightarrow \infty}\left|\zeta_{j}^{(n)}\right|^{2}=0$ or $B\left(h, f_{j}\right)=0 \quad \forall h \in \mathfrak{p}_{0}$ (i.e. $\left.f_{j} \in \mathfrak{p}_{0}^{\prime}\right)$. Now we can rewrite the argument around Eqn. (12) almost verbatim to obtain $\mathcal{O}_{2}=\mathrm{C}^{*}\left(\delta_{\mathfrak{p}_{0}^{\prime}}\right)+\mathcal{D}_{2}$.

\subsection{Claim $\mathfrak{p}_{0}^{\prime}=\mathfrak{p}$.}

Proof: Clearly $\mathfrak{p}_{0}^{\prime} \supseteq \mathfrak{p}$ by definition. For the converse inclusion, let $f \in C_{c}^{\infty}\left(\mathbb{R}^{4}, \mathbb{R}^{4}\right)$ such that $\rho(f):=\widehat{f} \mid C_{+} \in \mathfrak{p}_{0}^{\prime}$, i.e. we have $B(\rho(f), \rho(k))=0 \forall \rho(k) \in \mathfrak{p}_{0}$. Now by Proposition 5.12, if $\rho(k)$ is in $\mathfrak{p}_{0}$, then $\rho(k)_{\mu}(p)=i p_{\mu} h(p)$ for some $h$ such that $\rho(k) \in \mathfrak{Z}_{(0)}$. In particular, we can take $h=\rho(r)$ for $r \in C_{c}^{\infty}\left(\mathbb{R}^{4}, \mathbb{R}\right)$, in which case $\rho(k)_{\mu}=\rho\left(\partial_{\mu} r\right)$, and so

$$
\begin{aligned}
0 & =B(\rho(f), \rho(k))=\widehat{D}(f, k) \\
& =\iint f_{\mu}(x) k^{\mu}(y) D(x-y) d^{4} x d^{4} y \\
& =\iint f_{\mu}(z+y) k^{\mu}(y) D(z) d^{4} z d^{4} y \\
& =\int\left(\int f_{\mu}(z+y) \frac{\partial r(y)}{\partial y_{\mu}} d^{4} y\right) D(z) d^{4} z
\end{aligned}
$$




$$
\begin{aligned}
& =-\int\left(\int\left(\partial^{\mu} f_{\mu}\right)(z+y) r(y) d^{4} y\right) D(z) d^{4} z \\
& =-\iint\left(\partial^{\mu} f_{\mu}\right)(x) r(y) D(x-y) d^{4} y d^{4} x \\
& =-\widetilde{D}\left(\rho\left(\partial^{\mu} f_{\mu}\right), \rho(r)\right)
\end{aligned}
$$

for all $r \in C_{c}^{\infty}\left(\mathbb{R}^{4}, \mathbb{R}\right)$ and where $D$ is the Pauli-Jordan distribution and $\widetilde{D}$ is the symplectic form for the free neutral scalar bosonic field. It is well-known that $\widetilde{D}$ is nondegenerate on $\rho\left(C_{c}^{\infty}\left(\mathbb{R}^{4}, \mathbb{R}\right)\right.$ ), (to see this, use the Schwartz density argument in the proof of Proposition 5.12) hence since $\rho\left(\partial^{\mu} f_{\mu}\right)$ is also in this space, we conclude $\rho\left(\partial^{\mu} f_{\mu}\right)=0$, i.e. $p^{\mu} \rho\left(f_{\mu}\right)=0$, i.e. by Eqn. (8) $\rho(f) \in \mathfrak{p}$. Hence we have the reverse inclusion, so $\mathfrak{p}_{0}^{\prime}=\mathfrak{p}$.

Thus $\mathcal{O}_{2}=\mathrm{C}^{*}\left(\delta_{\mathfrak{p}}\right)+\mathcal{D}_{2}$ and so $\mathcal{R}_{2} \cong \mathcal{R}_{(2)}$.

\subsection{The weak spectral condition}

A very important additional property which is used in the analysis of algebraic QFT, is that of the spectral condition.

6.7 Definition An action $\alpha: \mathcal{P}_{+}^{\uparrow} \rightarrow$ Aut $\mathcal{F}_{0}$ on a $C^{*}$-algebra $\mathcal{F}_{0}$ satisfies the spectral condition if there is a state $\omega \in \mathfrak{S}\left(\mathcal{F}_{0}\right)$ such that

(i) $\omega$ is translation-invariant, i.e. $\omega \circ \alpha_{g}=\omega \forall g \in \mathbb{R}^{4} \subset \mathcal{P}_{+}^{\uparrow}$,

(ii) the spectrum of the generators of translations in $\pi_{\omega}$ is in the forward light cone $V_{+}$.

Let now $\Theta \rightarrow(\mathcal{F}(\Theta), \mathcal{U}(\Theta))$ be a system of local constraints with reduction isotony and weak covariance. We want to find the weakest requirement on $\Theta \rightarrow(\mathcal{F}(\Theta), \mathcal{U}(\Theta))$ to ensure that $\widetilde{\alpha}: \mathcal{P}_{+}^{\uparrow} \rightarrow$ Aut $\mathcal{R}_{0}$ (cf. Theorem 3.14(iii)) satisfies the spectral condition. We propose:

6.8 Definition The given action $\alpha: \mathcal{P}_{+}^{\uparrow} \rightarrow$ Aut $\mathcal{F}_{0}$ on $\mathcal{F}_{0}=\underline{\lim } \mathcal{F}(\Theta)$ satisfies the weak spectral condition iff the set

$$
\mathcal{C}:=\left(\mathcal{U}_{e}-\mathbb{1}\right) \cup\left\{\alpha_{f}(A) \mid A \in \mathcal{O}_{0}, f \in F\left(V_{+}\right)\right\} \subset \mathcal{O}_{0}:=\underset{\lim }{\longrightarrow} \mathcal{O}(\Theta)
$$

is a first-class constraint set, where we used the notation

$$
\begin{aligned}
\mathcal{U}_{e} & :=\bigcup_{\Theta \in \Gamma} \mathcal{U}(\Theta), \quad \alpha_{f}(A):=\int_{\mathbb{R}^{4}} \alpha_{t}(A) f(t) d^{4} t \\
F\left(V_{+}\right) & :=\left\{f \in L^{1}\left(\mathbb{R}^{4}\right) \mid \operatorname{supp} \widehat{f} \subset \mathbb{R}^{4} \backslash V_{+} \quad \text { and } \operatorname{supp} \widehat{f} \text { is compact }\right\}
\end{aligned}
$$

where $\widehat{f}$ denotes the Fourier transform of $f$.

6.9 Theorem Let $\Theta \rightarrow(\mathcal{F}(\Theta), \mathcal{U}(\Theta))$ be a local system of contraints with reduction isotony and weak covariance. Then $\widetilde{\alpha}: \mathcal{P}_{+}^{\uparrow} \rightarrow$ Aut $\mathcal{R}_{0}$ satisfies the spectral condition iff $\alpha: \mathcal{P}_{+}^{\uparrow} \rightarrow$ Aut $\mathcal{F}_{0}$ satisfies the weak spectral condition.

Proof: We will use the notation in Subsection 6.1 By the last definition, $\alpha$ satisfies the weak spectral condition iff $\mathcal{C}$ is first-class iff the left ideal $\left[\mathcal{O}_{0} \mathcal{C}\right]$ in $\mathcal{O}_{0}$ is proper iff the left ideal $\xi_{0}\left(\left[\mathcal{O}_{0} \mathcal{C}\right]\right)=\left[\mathcal{R}_{0} \xi_{0}(\mathcal{C})\right]$ is proper in $\mathcal{R}_{0}$ where $\xi_{0}: \mathcal{O}_{0} \rightarrow \mathcal{O}_{0} /\left(\mathcal{D}_{e} \cap \mathcal{O}_{0}\right)=\mathcal{R}_{0}$ is the canonical factoring map, and we used $\operatorname{Ker} \xi_{0}=\mathcal{D}_{e} \cap \mathcal{O}_{0} \subset\left[\mathcal{O}_{0}\left(\mathcal{U}_{e}-\mathbb{1}\right)\right] \subset\left[\mathcal{O}_{0} \mathcal{C}\right]$. Now 
$\xi_{0}(\mathcal{C})=\left\{\xi_{0}\left(\alpha_{f}(A)\right) \mid A \in \mathcal{O}_{0}, f \in F\left(V_{+}\right)\right\}$because $\xi_{0}\left(\mathcal{U}_{e}-\mathbb{1}\right)=0$. Let $A \in \mathcal{O}_{0}$ and $f \in F\left(V_{+}\right)$, then

$$
\begin{aligned}
\xi_{0}\left(\alpha_{f}(A)\right) & =\xi_{0}\left(\int_{\mathbb{R}^{4}} \alpha_{t}(A) f(t) d^{4} t\right)=\int_{\mathbb{R}^{4}} \xi_{0}\left(\alpha_{t}(A)\right) f(t) d^{4} t \\
& =\int_{\mathbb{R}^{4}} \widetilde{\alpha}_{t}\left(\xi_{0}(A)\right) f(t) d^{4} t=\widetilde{\alpha}_{f}\left(\xi_{0}(A)\right) .
\end{aligned}
$$

Thus $\left[\mathcal{R}_{0} \xi_{0}(\mathcal{C})\right]$ is the left ideal generated in $\mathcal{R}_{0}$ by $\left.\left\{\widetilde{\alpha}_{f}(B)\right) \mid B \in \mathcal{R}_{0}, f \in F\left(V_{+}\right)\right\}$and this is precisely Doplicher's left ideal (cf. [35, 36]), which is proper iff $\widetilde{\alpha}$ satisfies the spectral condition by 2.7.2 in Sakai [36].

In general the weak spectral condition seems very difficult to verify directly. However, for the Gupta-Bleuler example in this paper, it is easily verified via the last theorem:

Example. We show that the final HK-QFT of the Gupta-Bleuler example (as expressed in Theorem 5.14) satisfies the spectral condition, and hence the initial system must satisfy the weak spectral condition. Thus we need to show the existence of a state on $\widetilde{\mathcal{R}}_{0}:=\lim \widetilde{\mathcal{R}}(\Theta)$ which satisfies the two conditions in Definition 6.7 for the action $\widetilde{\alpha}: \mathcal{P}_{+}^{\uparrow} \rightarrow$ Aut $\widetilde{\mathcal{R}}_{0}$. Now the usual Gupta-Bleuler theory studied in Subsection 5.6 produced a Fock state $\omega_{0}$ on $\widetilde{\mathcal{R}}_{0}=\overline{\Delta\left(\mathfrak{p} / \mathfrak{p}_{0}, \widetilde{B}\right)}$ given by the formula

$$
\omega_{0}\left(\delta_{\zeta(f)}\right):=\exp (-K(f, f) / 4), \quad f \in \mathfrak{p}
$$

where $\zeta: \mathfrak{p} \rightarrow \mathfrak{p} / \mathfrak{p}_{0}$ is the usual factoring map, and we want to show that it satisfies the spectral condition. We already know that $K$ produces a Hilbert inner product on $\mathfrak{p} / \mathfrak{p}_{0}$, and that it is Poincaré invariant w.r.t. $\widetilde{V}_{g}$ which denotes the factoring of $V_{g}$ to $\mathfrak{p} / \mathfrak{p}_{0}$. Thus the implementer of a Poincaré transformation $g$ is just the second quantization of $\widetilde{V}_{g}$, i.e. $U_{g}:=\Gamma\left(\widetilde{V}_{g}\right)$. We need to verify the spectral condition for the generators of the translations. Recall for $g=(\Lambda, a)$ we have $\left(V_{g} f\right)(p)=e^{-i p a} \Lambda f\left(\Lambda^{-1} p\right)$. Translation by a therefore acts by multiplication operators $\left(V_{a} f\right)(p)=e^{-i p a} f(p)$ with infinitesimal generators $\widetilde{P}_{\mu}$ of $\widetilde{V}_{a}$ being the factoring to $\mathfrak{p} / \mathfrak{p}_{0}$ of the multiplication operators $f(p) \rightarrow p_{\mu} f(p)$. Now for $f \in \mathfrak{p}$ :

$$
K\left(\zeta(f), \widetilde{P}_{0} \zeta(f)\right)=-2 \pi \int_{C_{+}} \overline{f^{\mu}(p)} p_{0} f_{\mu}(p) \frac{d^{3} p}{p_{0}}=-2 \pi \int_{C_{+}} \overline{f^{\mu}(p)} f_{\mu}(p) d^{3} p \geq 0
$$

since we have shown in the proof of Proposition 5.20 that $\overline{f^{\mu}(p)} f_{\mu}(p) \leq 0 \quad \forall f \in \mathfrak{p}, p \in C_{+}$. So $\widetilde{P}_{0} \geq 0$. Since $U_{a}=\Gamma\left(\widetilde{V}_{a}\right)=\exp \left(-i a_{\mu} d \Gamma\left(\widetilde{P}^{\mu}\right)\right)$, the generators for translation for $\omega$ are $d \Gamma\left(\widetilde{P}^{\mu}\right)$, and so $\widetilde{P}_{0} \geq 0$ implies $d \Gamma\left(\widetilde{P}_{0}\right) \geq 0$.

To conclude, notice from the fact that $\widetilde{P}_{\mu}$ acts on $\mathfrak{p} / \mathfrak{p}_{0}$ and in $\mathfrak{p}$ we have restriction to $C_{+}$, that the spectrum of $\widetilde{P}_{\mu}$ must be in $C_{+}$. Now in second quantization on an n-particle space:

$$
d \Gamma\left(\widetilde{P}_{\mu}\right)=\widetilde{P}_{\mu} \otimes \mathbb{1} \otimes \cdots \otimes \mathbb{1}+\mathbb{1} \otimes \widetilde{P}_{\mu} \otimes \mathbb{1} \otimes \cdots \otimes \mathbb{1}+\cdots+\mathbb{1} \otimes \cdots \otimes \mathbb{1} \otimes \widetilde{P}_{\mu}
$$

and the spectrum for this will be all possible sums of $n$ vectors in $C_{+}$, and this will always be in $V_{+}$. Since the spectrum for the full $d \Gamma\left(\widetilde{P}_{\mu}\right)$ is the sum over all those on the $\mathrm{n}$-particle spaces, this will still be in $V_{+}$since $V_{+}$is a cone. Thus we have verified the spectral condition as claimed.

\section{Conclusions.}

In this paper we introduced the concept of a system of local quantum constraints and we obtained a "weak" version of each of the Haag-Kastler axioms of isotony, causality, covariance and spectrality in such a way that after a local constraining procedure the resulting system of physical algebras satisfies the usual version of these axioms. We analyzed Gupta-Bleuler electromagnetism 
in detail and showed that it satisfies these weak axioms, but that it violates the causality axiom. This example was particularly satisfying, in that we obtained by pure $\mathrm{C}^{*}$-algebra techniques the correct physical algebra and positive energy Fock-representation without having to pass through an indefinite metric representation. We did however also point out the precise connection with the usual indefinite metric representation.

There are some further aspects of our Gupta-Bleuler example which are of independent interest. These are:

(1) the use of nonlinear constraints $\chi(h)^{\dagger} \chi(h)$, which we realised as automorphisms on the field algebra (outer constraint situation),

(2) a nonstandard extension of our smearing formulii to complex-valued functions (cf. Remark 5.4(ii)), which implied noncausal behaviour on nonphysical objects, where the latter were eliminated in the final theory (hence the need for weak causality),

(3) the use of nonregular representations, but as in the last point the nonregularity was restricted to nonphysical objects.

There are many future directions of development for this project, and a few of the more evident ones are:

- Find an example of a realistic constrained local field theory which satisfies the weak HaagKastler axioms, but violates the usual covariance axiom (a variant of the Coulomb gauge may work).

- Continue the analysis here for the rest of the Haag-Kastler axioms, i.e. find the appropriate weak versions of e.g. the axioms of additivity, local normality, local definiteness etc., as well as examples which satisfy the weak axioms but not the usual ones.

- In the present paper we assumed a system of local constraints which is first-class. Now a reduction procedure at the $\mathrm{C}^{*}$-level exists also for second class constraints (cf. [10]) and so one can therefore ask what the appropriate weakened form of the Haag-Kastler axioms should be for such a system. A possible example for such an analysis is electromagnetism in the Coulomb gauge.

- Develop a QFT example with nonlinear constraints. This is related to the difficulty of abstractly defining the $\mathrm{C}^{*}$-algebra of a QFT with nontrivial interaction. Our GuptaBleuler example has several similarities with Dimock's version of a Yang-Mills theory on the cylinder [37], and so this seems to be a possible candidate for further development.

\section{Appendix 1}

Next we wish to gain further understanding of the algebras $\mathcal{D}, \mathcal{O}, \mathcal{R}$ by exploiting the hereditary property of $\mathcal{D}$. Denote by $\pi_{u}$ the universal representation of $\mathcal{F}$ on the universal Hilbert space $\mathcal{H}_{u}$ [9, Section 3.7]. $\mathcal{F}^{\prime \prime}$ is the strong closure of $\pi_{u}(\mathcal{F})$ and since $\pi_{u}$ is faithful we make the usual identification of $\mathcal{F}$ with a subalgebra of $\mathcal{F}^{\prime \prime}$, i.e. generally omit explicit indication of $\pi_{u}$. If $\omega \in \mathfrak{S}(\mathcal{F})$, we will use the same symbol for the unique extension of $\omega$ from $\mathcal{F}$ to $\mathcal{F}^{\prime \prime}$.

8.1 Theorem For a constrained system $(\mathcal{F}, \mathcal{C})$ there exists a projectionP $\in \mathcal{F}^{\prime \prime}$ such that

(i) $\mathcal{N}=\mathcal{F}^{\prime \prime} P \cap \mathcal{F}$,

(ii) $\mathcal{D}=P \mathcal{F}^{\prime \prime} P \cap \mathcal{F}$ and 
(iii) $\mathfrak{S}_{D}=\{\omega \in \mathfrak{S}(\mathcal{F}) \mid \omega(P)=0\}$.

Proof: From Theorem 2.3 (i) $\mathcal{D}$ is a hereditary $\mathrm{C}^{*}$-subalgebra of $\mathcal{F}$ and by 3.11 .10 and 3.11 .9 in [9] there exists a projection $P \in \mathcal{F}^{\prime \prime}$ such that $\mathcal{D}=P \mathcal{F}^{\prime \prime} P \cap \mathcal{F}$. Further by the proof of Theorem 2.2 (iii) as well as 3.10.7 and 3.11.9 in [9] we obtain that $\mathcal{N}=\mathcal{F}^{\prime \prime} P \cap \mathcal{F}$ and

$$
\mathfrak{S}_{D}=\{\omega \in \mathfrak{S}(\mathcal{F}) \mid \omega(P)=0\}
$$

which concludes this proof.

A projection satisfying the conditions of Theorem 8.1 is called open in 9].

8.2 Theorem Let $P$ be the open projection in Theorem 8.1. Then:

(i) $\mathcal{O}=\{A \in \mathcal{F} \mid P A(\mathbb{1}-P)=0=(\mathbb{1}-P) A P\}=P^{\prime} \cap \mathcal{F}$, and

(ii) $\mathcal{C}^{\prime} \cap \mathcal{F} \subset \mathcal{O}$.

Proof: (i) Recall that $\mathcal{O}=M_{\mathcal{F}}(\mathcal{D})$, and let $A \in \mathcal{F}$ and $D \in \mathcal{D}$. Then by Theorem 8.1 there exists an $F \in \mathcal{F}^{\prime \prime}$ such that $D=P F P$ and so

$$
\begin{aligned}
A D & =(P A P+(\mathbb{1}-P) A P+P A(\mathbb{1}-P)+(\mathbb{1}-P) A(\mathbb{1}-P)) P F P \\
& =P A P F P+(\mathbb{1}-P) A P F P \\
& =P A P D+(\mathbb{1}-P) A P D .
\end{aligned}
$$

Therefore using Theorem 8.1 again we have $A D \in \mathcal{D}$ for all $D \in \mathcal{D}$ iff $(\mathbb{1 1}-P) A P D=0$ for all $D \in \mathcal{D}$. But from 3.11.9 in [9] $P$ is in the strong closure of $\mathcal{D}$ in $\mathcal{F}^{\prime \prime}$ so that $A D \in \mathcal{D} \forall D \in \mathcal{D}$ iff $(\mathbb{1}-P) A P=0$. Taking adjoints we get also the condition $P A(\mathbb{1}-P)=0$ iff $\mathcal{D} A \subset \mathcal{D}$.

(ii) Let $D \in \mathcal{D}=[\mathcal{F C}] \cap[\mathcal{C F}]$ and $A \in \mathcal{C}^{\prime} \cap \mathcal{F}$. Then $A D \in[\mathcal{F C}] \cap[A \mathcal{C F}]=[\mathcal{F C}] \cap[\mathcal{C} A \mathcal{F}] \subset$ $\mathcal{D}$. Similarly, $D A \in \mathcal{D}$ so that by definition we have $A \in \mathcal{O}$.

What these two last theorems mean, is that with respect to the decomposition

$$
\mathcal{H}_{u}=P \mathcal{H}_{u} \oplus(\mathbb{1}-P) \mathcal{H}_{u}
$$

we may rewrite

$$
\begin{aligned}
& \mathcal{D}=\left\{F \in \mathcal{F} \mid F=\left(\begin{array}{ll}
D & 0 \\
0 & 0
\end{array}\right), D \in P \mathcal{F} P\right\} \text { and } \\
& \mathcal{O}=\left\{F \in \mathcal{F} \mid F=\left(\begin{array}{cc}
A & 0 \\
0 & B
\end{array}\right), A \in P \mathcal{F} P, B \in(\mathbb{1}-P) \mathcal{F}(\mathbb{1}-P)\right\} .
\end{aligned}
$$

It is clear that in general $\mathcal{O}$ can be much greater than the traditional observables $\mathcal{C}^{\prime} \cap \mathcal{F}$. Next we show how to identify the final algebra of physical observables $\mathcal{R}$ with a subalgebra of $\mathcal{F}^{\prime \prime}$.

8.3 Theorem For $P$ as above we have:

$$
\mathcal{R} \cong\left\{F \in \mathcal{F} \mid F=\left(\begin{array}{ll}
0 & 0 \\
0 & A
\end{array}\right)\right\}=(\mathbb{1}-P)\left(P^{\prime} \cap \mathcal{F}\right) \subset \mathcal{F}^{\prime \prime} .
$$

Proof: The homomorphism $\Phi: \mathcal{O} \rightarrow(\mathbb{1}-P)\left(P^{\prime} \cap \mathcal{F}\right)$ defined by $\Phi(A):=(\mathbb{1}-P) A, A \in$ $\mathcal{O}=P^{\prime} \cap \mathcal{F}$, will establish an isomorphism with $\mathcal{R}:=\mathcal{O} / \mathcal{D}$ if we can show that $\operatorname{Ker} \Phi=\mathcal{D}$, i.e. (11-P) $A=0$ iff $A \in \mathcal{D}$. Clearly, if $A \in \mathcal{D}=P \mathcal{F}^{\prime \prime} P \cap \mathcal{F}$ (cf. Theorem 8.1), then $(\mathbb{1}-P) A=0$. Conversely, assume that $A \in \mathcal{O}=P^{\prime} \cap \mathcal{F}$ satisfies $(\mathbb{1 l}-P) A=0$, i.e. $A=P A$. Then, $A \in P \mathcal{F}^{\prime \prime} \cap \mathcal{F}$ and so since $A \in P^{\prime} \cap \mathcal{F}$, we have $A \in P \mathcal{F}^{\prime \prime} P \cap \mathcal{F}=\mathcal{D}$, which ends the proof. 
8.4 Remark (i) With the preceding result we may interpret the projection $P$ in Theorem 8.1 as being equivalent to the set $\mathcal{C}$ if we are willing to enlarge $\mathcal{F}$ to $\mathrm{C}^{*}(\mathcal{F} \cup\{P\})$. This can be partially justified by the fact that

$$
\mathcal{R} \cong(\mathbb{1}-P)\left(P^{\prime} \cap \mathcal{F}\right) \subset \mathrm{C}^{*}(\mathcal{F} \cup\{P\})
$$

(ii) The projection $P$ can also be used to make contact with the original heuristic picture. Given a Dirac state $\omega \in \mathfrak{S}_{D}$ we see from Theorem 8.1 (iii) that $\mathbb{1}-\pi_{\omega}(P)$ is the projection onto the physical subspace

$$
\mathcal{H}_{\omega}^{(p)}:=\left\{\psi \in \mathcal{H}_{\omega} \mid \pi_{\omega}(\mathcal{C}) \psi=0\right\}
$$

Since $\pi_{\omega}(\mathcal{O}) \subset \pi_{\omega}(P)^{\prime} \cap \pi_{\omega}(\mathcal{F})$, then $\pi_{\omega}(\mathcal{O})$ is a subalgebra of the algebra of observables in the field algebra $\pi_{\omega}(\mathcal{F})$ which preserves the physical subspace. (In fact $\mathcal{O}=\{F \in \mathcal{F} \mid$ $\left.\left.\pi_{\omega}(F) \mathcal{H}_{\omega}^{(p)} \subseteq \mathcal{H}_{\omega}^{(p)} \quad \forall \omega \in \mathfrak{S}(\mathcal{F})\right\}\right)$. If $\pi_{\omega}$ is faithful, then $\pi_{\omega}(\mathcal{O})$ contains the traditional observables $\pi_{\omega}(\mathcal{C})^{\prime} \cap \pi_{\omega}(\mathcal{F})$. Now restricting $\pi_{\omega}(\mathcal{O})$ to the subspace $\mathcal{H}_{\omega}^{(p)}=\left(\mathbb{1}-\pi_{\omega}(P)\right) \mathcal{H}_{\omega}$ we have for the final constrained system:

$$
\pi_{\omega}(\mathcal{O})\left\lceil\mathcal{H}_{\omega}^{(p)}=\left(\mathbb{1}-\pi_{\omega}(P)\right) \pi_{\omega}(\mathcal{O})=\pi_{\omega}\left((\mathbb{1}-P)\left(P^{\prime} \cap \mathcal{F}\right)\right),\right.
$$

which by Theorem 8.3 produces a representation of $\mathcal{R}$.

\section{Appendix 2}

We will give in this appendix the proof of Theorem 4.4. Recall the notation and results of Subsection 4.2 .

8.5 Theorem Given nondegenerate $(\mathfrak{X}, B)$ and $\mathfrak{s} \subset \mathfrak{X}$ as in Subsection $4 . \mathfrak{2}$, where $\mathfrak{s} \subset \mathfrak{s}^{\prime}$ and $\mathfrak{s}=\mathfrak{s}^{\prime \prime}$, then

$$
\mathcal{O}=\mathrm{C}^{*}\left(\delta_{\mathfrak{s}^{\prime}} \cup \mathcal{D}\right)=\left[\mathrm{C}^{*}\left(\delta_{\mathfrak{s}^{\prime}}\right) \cup \mathcal{D}\right] .
$$

Proof: From Theorem 2.3 (ii) and (v) it is clear that $\mathcal{O} \supset \mathrm{C}^{*}\left(\delta_{\mathfrak{s}^{\prime}} \cup \mathcal{D}\right)$ and we only have to prove the converse inclusion. We first show that for any Dirac state $\omega$ of $\overline{\Delta(\mathfrak{X}, B)}$ we have that $\pi_{\omega}\left(\delta_{f}\right) \Omega_{\omega} \perp \mathcal{H}_{\omega}^{(p)}:=\left\{\psi \in \mathcal{H}_{\omega} \mid \pi_{\omega}\left(\delta_{\mathfrak{s}}\right) \psi=\psi\right\}$ for all $f \notin \mathfrak{s}^{\prime}$. For any $f \notin \mathfrak{s}^{\prime}$, choose a $k \in \mathfrak{s}$ such that $B(f, k) \notin 2 \pi \mathbb{Z}$. Then

$$
\pi_{\omega}\left(\delta_{k}\right)\left(\pi_{\omega}\left(\delta_{f}\right) \Omega_{\omega}\right)=e^{i B(k, f)} \pi_{\omega}\left(\delta_{f} \delta_{k}\right) \Omega_{\omega}=e^{i B(k, f)} \pi_{\omega}\left(\delta_{f}\right) \Omega_{\omega}
$$

and so $\pi_{\omega}\left(\delta_{f}\right) \Omega_{\omega}$ is in a different eigenspace of $\pi_{\omega}\left(\delta_{k}\right)$ than $\mathcal{H}_{\omega}^{(p)}$, hence must be orthogonal to it.

Now recall (cf. Remark 8.4 (ii)) that if $A \in \mathcal{O}$, then $\pi_{\omega}(A)$ preserves $\mathcal{H}_{\omega}^{(p)}$ (here $\omega$ is a Dirac state). Thus $\pi_{\omega}(A) \Omega_{\omega} \perp \pi_{\omega}\left(\delta_{f}\right) \Omega_{\omega}$ for $f \notin \mathfrak{s}^{\prime}$, and so $\omega\left(\delta_{f} A\right)=0$ for $f \notin \mathfrak{s}^{\prime}$. Now let $A_{n}=\sum_{i=1}^{N_{n}} \lambda_{i}^{(n)} \delta_{h_{i}} \subset \Delta(\mathfrak{X}, B)$ be a sequence converging to $A$. We furthermore partition the set $\left\{h_{i} \mid i \in \mathbb{N}\right\}$ into $\mathfrak{s}$-equivalence classes and choose one representative in each, so that we can write

$$
A_{n}=\sum_{j} \delta_{h_{j}} \sum_{i} \beta_{i j}^{(n)} \delta_{c_{i j}} \quad \text { where } \quad c_{i j} \in \mathfrak{s} \quad \text { and } \quad h_{j}-h_{k} \notin \mathfrak{s} \quad \text { if } \quad j \neq k
$$

i.e. the first sum is over representatives in different equivalence classes. Thus we get for $A \in \mathcal{O}$ that for all $f \notin \mathfrak{s}^{\prime}$ :

$$
0=\lim _{n \rightarrow \infty} \omega\left(\delta_{f} A_{n}\right)=\lim _{n \rightarrow \infty} \sum_{j, i} \beta_{i j}^{(n)} \omega\left(\delta_{f} \delta_{h_{j}} \delta_{c_{i j}}\right) .
$$


Now we make a particular choice for $\omega$, by setting $\omega\left(\delta_{f}\right)=1$ if $f \in \mathfrak{s}$ and zero otherwise. (To

see that this indeed defines a state, note that if we factors the central state on $\overline{\Delta\left(\mathfrak{s}^{\prime} / \mathfrak{s}^{\prime \prime}, \widetilde{B}\right)}$ to $\mathrm{C}^{*}\left(\delta_{\mathfrak{s}^{\prime}}\right)$, then by [3, p. 387] it extends uniquely to $\overline{\Delta(\mathfrak{X}, B)}$ and will coincide with $\left.\omega\right)$. Then

$$
\begin{aligned}
\omega\left(\delta_{f} \delta_{h_{j}} \delta_{c_{i j}}\right) & =\exp \left[i\left(B\left(f, h_{j}\right)+B\left(f+h_{j}, c_{i j}\right)\right) / 2\right] \omega\left(\delta_{f+h_{j}+c_{i j}}\right) \\
& =e^{\frac{i}{2} B\left(f, h_{j}\right)} \chi_{\mathfrak{s}}\left(f+h_{j}\right)
\end{aligned}
$$

where $\chi_{\mathfrak{s}}$ denotes the characteristic function of the set $\mathfrak{s}$. Thus Eqn. (25) becomes:

$$
\lim _{n \rightarrow \infty} \sum_{j, i} \beta_{i j}^{(n)} e^{\frac{i}{2} B\left(f, h_{j}\right)} \chi_{\mathfrak{s}}\left(f+h_{j}\right)=0 \quad \forall f \notin \mathfrak{s}^{\prime} .
$$

If there is a $k$ such that $h_{k} \notin \mathfrak{s}^{\prime}$, we can choose $f=-h_{k}$ in the previous equation, then since $h_{j}-h_{k} \notin \mathfrak{s}$ for $j \neq k$, we get $\chi_{\mathfrak{s}}\left(h_{j}-h_{k}\right)=\delta_{j k}$ and thus $\lim _{n \rightarrow \infty} \sum_{i} \beta_{i k}^{(n)}=0$.

Consider now the universal representation $\pi_{u}: \mathcal{A} \rightarrow \mathcal{B}\left(\mathcal{H}_{u}\right)$ and let $\psi \in \mathcal{H}_{u}^{(p)}:=\left\{\psi \in \mathcal{H}_{u}^{(p)} \mid\right.$ $\left.\pi_{u}(\mathcal{D}) \psi=0\right\}$. Then for each $k$ such that $h_{k} \notin \mathfrak{s}^{\prime}$ we have

$$
\lim _{n} \pi_{u}\left(\delta_{h_{k}} \sum_{i} \beta_{i k}^{(n)} \delta_{c_{i k}}\right) \psi=\pi_{u}\left(\delta_{h_{k}}\right) \lim _{n} \sum_{i} \beta_{i k}^{(n)} \psi=0
$$

Hence in the sums involved in $\pi_{u}\left(A_{n}\right) \psi$ we can drop all terms where $h_{j} \notin \mathfrak{s}^{\prime}$, i.e.

$$
\begin{aligned}
\pi_{u}(A) \psi & =\lim _{n} \pi_{u}\left(A_{n}\right) \psi=\lim _{n} \sum_{h_{j} \in \mathfrak{s}^{\prime}} \pi_{u}\left(\delta_{h_{j}}\right) \sum_{i} \beta_{i k}^{(n)} \pi_{u}\left(\delta_{c_{i k}}\right) \psi \\
& =\lim _{n} \sum_{h_{j} \in \mathfrak{s}^{\prime}} \pi_{u}\left(\delta_{h_{j}}\right) \sum_{i} \beta_{i k}^{(n)} \psi \in \pi_{u}\left(\mathrm{C}^{*}\left(\delta_{\mathfrak{s}^{\prime}}\right)\right) \psi \quad \forall \psi \in \mathcal{H}_{u}^{(p)} .
\end{aligned}
$$

Thus the restriction of $\mathcal{O}=\pi_{u}(\mathcal{O})$ to $\mathcal{H}_{u}^{(p)}$ is the same as the restriction of $\mathrm{C}^{*}\left(\delta_{\mathfrak{s}^{\prime}}\right)$ to $\mathcal{H}_{u}^{(p)}$ (given that $\left.\mathrm{C}^{*}\left(\delta_{\mathfrak{s}^{\prime}}\right) \subset \mathcal{O}\right)$. However, recall from Theorem 8.3 and the preceding remarks, that $\mathcal{O} / \mathcal{D}=$ $\mathcal{R} \cong \mathcal{O} \mid \mathcal{H}_{u}^{(p)}=\mathrm{C}^{*}\left(\delta_{\mathfrak{s}^{\prime}}\right)\left\lceil\mathcal{H}_{u}^{(p)}\right.$ (with respect to the open projection $P$, we have $\left.\mathcal{H}_{u}^{(p)}=(\mathbb{1}-P) \mathcal{H}_{u}\right)$. Thus $\mathcal{O}=\mathrm{C}^{*}\left(\delta_{\mathfrak{s}^{\prime}}\right)+\mathcal{D}$.

\section{Acknowledgements.}

We are both very grateful to Prof. D. Buchholz for the friendly interest which he took in this paper, and for pointing out an important mistake in an earlier version of it. One of us (H.G.) would like to thank the Erwin Schrödinger Institute for Mathematical Physics in Vienna (where a substantial part of this work was done) for their generous assistance, as well as Prof. H. Baumgärtel for his warm hospitality at the University of Potsdam, where this project was started. We thank the $\mathrm{sfb} 288$ for support during this visit. We also benefitted from an ARC grant which funded a visit of F.Ll. to the University of New South Wales. Finally, F.Ll. would like to thank Hanno Gottschalk and Wolfgang Junker for helpful conversations, as well as Sergio Doplicher for his kind hospitality at the 'Dipartamento di Matematica dell' Università di Roma 'La Sapienza" in March 2000, when the final version of this paper was prepared. The visit was supported by a EU TMR network "Implementation of concept and methods from Non-Commutative Geometry to Operator Algebras and its applications", contract no. ERB FMRX-CT 96-0073. 


\section{References}

[1] Haag, R., Kastler, D.: An algebraic approach to quantum field theory. J. Math. Phys. 5, 848-861 (1964)

[2] Haag, R.: Local Quantum Physics. Berlin: Springer Verlag 1992

[3] Grundling, H., Hurst, C.A.: Algebraic quantization of systems with a gauge degeneracy. Commun. Math. Phys. 98, 369-390 (1985)

[4] Doplicher, S., Haag, R., Roberts, J.E.: Fields, observables and gauge transformations I. Commun. Math. Phys. 13, 1-23 (1969)

[5] Landsman, N.P.: Rieffel induction as generalised quantum Marsden-Weinstein reduction. J. Geom. Phys. 15, 285-319 (1995)

Grundling, H., Hurst, C.A.: Constrained dynamics for quantum mechanics I. J. Math. Phys. 39, 3091-3119 (1998)

M. Henneaux, C. Teitelboim: Quantization of Gauge Systems. Princeton University Press, Princeton 1992

Giulini, D., Marolf, D.: On the generality of refined algebraic quantization. gr-qc/9812024

Klauder, J., Ann. Physics 254, 419-453 (1997)

Faddeev, L., Jackiw, R.: Hamiltonian reduction of unconstrained and constrained systems. Phys. Rev. Lett. 60, 1692 (1988)

Landsman, N.P., Wiedemann, U.: Massless particles, electromagnetism and Rieffel induction. Rev. Math. Phys. 7, 923-958 (1995)

[6] Carey, A.L., Hurst, C.A.: Application of an algebraic quantization of the electromagnetic field. J. Math. Phys. 20, 810-819 (1979)

Ferrari, R., Picasso, L.E., Strocchi, F.: Some remarks on local operators in quantum electrodynamics. Commun. Math. Phys. 35, 25-38 (1974)

[7] Strocchi, F., Wightman, A.S.: Proof of the charge superselection rule in local relativistic quantum field theory. J. Math. Phys. 15, 2198-2224 (1974) [Erratum: ibid. 17, 1930-1931 (1976)]

[8] Grundling, H.: Systems with outer constraints. Gupta-Bleuler electromagnetism as an algebraic field theory. Commun. Math. Phys. 114, 69-91 (1988)

[9] Pedersen, G.K.: $\mathrm{C}^{*}$-Algebras and their Automorphism Groups. London: Academic Press 1989

[10] Grundling, H., Hurst, C.A.: The quantum theory of second class constraints: Kinematics. Commun. Math. Phys. 119, 75-93 (1988) [Erratum: ibid. 122, 527-529 (1989)]

[11] Dirac, P.A.M.: Lectures on Quantum Mechanics. Belfer Graduate School of Science: Yeshiva University 1964

[12] Murphy, G.J.: C*-Algebras and Operator Theory. Boston: Academic Press 1990

[13] Grundling, H., Hurst, C.A.: Algebraic structures of degenerate systems and the indefinite metric. J. Math. Phys. 28, 559-572 (1987)

[14] Lledó, F.: A family of examples with quantum constraints. Lett. Math. Phys. 40, 223-234 (1997)

[15] _ _ Algebraic properties of massless free nets. University of Potsdam: Ph.D. thesis 1999 
[16] Takeda, Z., Inductive limit and infinite direct product of operator algebras. Tohoku Math. J. 7, 67-86 (1955)

[17] Strocchi, F.: Gauge problem in quantum field theory. Phys. Rev. 162, 1429-1438 (1967)

[18] _ : Gauge problem in quantum field theory. III. Quantization of Maxwell equations and weak local commutativity. Phys. Rev. D 2, 2334-2340 (1970)

[19] Barut, A.O., Rączka, R.: Properties of non-unitary zero mass induced representations of the Poincaré group on the space of tensor-valued functions. Ann. Inst. H. Poincaré 17, 111-118 (1972)

[20] Weinberg, S.: The Quantum Theory of Fields. Vol. I. Cambridge: Cambridge University Press 1995.

[21] Kadison, R.V., Ringrose, J.R.: Fundamentals of the Theory of Operator II. Orlando: Academic Press 1986

[22] Manuceau, J.: C ${ }^{*}$-algèbre de relations de commutation. Ann. Inst. H. Poincaré 8, 139-161 (1968)

[23] Manuceau, J., Sirugue, M., Testard, D., Verbeure, A.: The smallest C*-algebra for canonical commutations relations. Commun. Math. Phys. 32, 231-243 (1973)

[24] Grundling, H., Hurst, C.A.: A note on regular states and supplementary conditions. Lett. Math. Phys. 15, 205-212 (1988) [Errata: ibid. 17, 173-174 (1989)]

[25] Gupta, S.N.: The theory of longitudinal photons in quantum electrodynamics. Proc. Phys. Soc. A63, 681-691 (1950)

[26] Bleuler, K.: Eine neue Methode zur Behandlung der longitudinalen und skalaren Photonen. Helv. Phys. Acta 23, 567-586 (1950)

[27] Strocchi, F: Locality and covariance in QED and gravitation. General proof of Gupta-Bleuler type formulations. In: Brittin, W.E., (ed.) Mathematical Methods in Theoretical Physics. Proceedings, pp. 551-568. Boulder: Colorado Ass. Univ. Press 1973

[28] Takesaki, M.: Theory of operator algebras I. New York: Springer-Verlag 1979

[29] Reed, M., Simon, B.: Methods of Modern Mathematical Physics II. Fourier Analysis, SelfAdjointness. New York: Academic Press 1975

[30] Dimock, J.: Quantizated electromagnetic field on a manifold. Rev. Math. Phys. 4, 223-233 (1992)

[31] Dimock, J.: Algebras of local observables on a manifold. Commun. Math. Phys. 77, 219-228 (1980)

[32] Choquet-Bruhat, Y: Hyperbolic partial differential equations on a manifold. In: DeWitt, C.M. and Wheeler, J.A., (eds.) Battelles Rencontres, 1967 Lectures in Mathematics and Physics, pp. 84-106. New York: W.A. Benjamin, Inc. 1968

[33] Thirring, W., Narnhofer, H.: Covariant QED without indefinite metric. Rev. Math. Phys. special issue, 197-211 (1992)

[34] Mintchev, M.: Quantization in Indefinite Metric. J. Phys. A 13, 1841-1859 (1980) 
[35] Doplicher, S.: An algebraic spectrum condition. Commun. Math. Phys. 1, 1-5 (1965)

[36] Sakai, S.: Operator Algebras in Dynamical Systems. Cambridge: Cambridge University Press 1991

[37] Dimock, J.: Canonical quantization of Yang-Mills on a circle. Rev. Math. Phys. 8, 85-102 (1996) 